\title{
Adaptive Mortar Edge Element Methods in Electromagnetic Field Computation
}

\author{
Ronald H.W. Hoppe
}

\begin{abstract}
We consider adaptive mortar edge element discretizations in the numerical solution of the quasi-stationary limit of Maxwell's equations, also known as the eddy currents equations, in three space dimensions. The approach is based on the macro-hybrid variational formulation with respect to a geometrically conforming, non overlapping decomposition of the computational domain. Using adaptively generated hierarchies of locally quasi-uniform and shape regular simplicial triangulations of the subdomains, the edge element discretizations involve the lowest order curl-conforming edge elements of Nédélec's first family. Due to the occurrence of nonmatching grids at the interfaces of adjacent subdomains, weak continuity of the tangential traces across the skeleton of the decomposition is realized by appropriately chosen Lagrange multipliers.

The mortar edge element discretized problems give rise to the numerical solution of algebraic saddle point problems which is taken care of by a multilevel iterative solver featuring a hybrid smoother that involves an additional defect correction on the subspace of irrotational vector fields.

Particular emphasis is on mesh adaptivity which is provided on the basis of an efficient and reliable residual-type a posteriori error estimator that relies on a Helmholtz decomposition of the error into an irrotational and weakly solenoidal part.

As applications, we consider the computation of eddy currents in converter modules which are used as electric drives for high power electro motors. A further example addresses the numerical simulation of LWD (Logging While Drilling) tools used in oil exploration for in situ measurements of geological formations and thus illustrates that the developed algorithm can as well be used for the time-harmonic Maxwell equations.
\end{abstract}

\section{Contents}

1. Introduction

2. The Hilbert spaces $\mathbf{H}(\mathbf{c u r l} ; \Omega), \mathbf{H}(\operatorname{div} ; \Omega)$ and its trace spaces 3

3. Macrohybrid formulation of the eddy currents equation $\quad 7$

4. Mortar edge element approximations

1991 Mathematics Subject Classification. 65N55,65N50,65N30,78M10.

Key words and phrases. electromagnetic field computation, domain decomposition methods, mortar edge elements, multilevel iterative solvers, a posteriori error estimation. 
5. Multilevel iterative solution methods 20

6. Residual-type a posteriori error estimators 25

7. Numerical Results 40

References $\quad 46$

\section{Introduction}

During the past two decades, advanced finite element discretizations including adaptive techniques and efficient iterative solution methods have been the focus of intensive research in computational electromagnetics (cf., e.g., the recent monographs [19,48] and the survey article [37] for a detailed exposure as well as the proceedings [70] for a collection of state-of-the-art research articles).

As far as the issue of finite element discretizations is concerned, the systematic introduction ofr curl-conforming edge elements by Nédélec [49,50] and Bossavit [18, 19] has provided an adequate tool for a physically consistent numerical simulation of electromagnetic phenomena. Indeed, edge elements are better suited than standard nodal based finite elements which may give rise to severe stability problems due to the occurrence of spurious modes (see e.g. [15,17]). Besides the $h$ version of edge elements, p and hp versions have been studied as well (cf., e.g., [1,53,54]. Recent interest focuses on the development, analysis and implementation of discontinuous Galerkin methods (cf. [45, 51]).

Adaptive grid refinement techniques based on appropriate a posteriori error estimators have reached a certain amount of maturity, as it is documented by a series of monographs on this topic (see $[2,6,7,31,64]$ ). However, for edge element discretizations of Maxwell's equations, not that much work has been done. We note that efficient and reliable residual-type as well as hierarchical a posteriori error estimators for the eddy currents equations have been developed and rigorously analyzed in [9] and [10].

For the appropriate numerical solution of large scale finite element discretized partial differential equations and systems thereof, multilevel techniques and domain decomposition methods belong to the most efficient algorithmic tools (cf., e.g., $[22,28,35,46,52,61,66])$. In the framework of computational electromagnetics, the most advanced geometric-type multigrid methods have been developed in $[5,36]$ (cf. also the survey article [8]), whereas a powerful algebraic multigrid approach has been provided in [59] (see also [34]). In the area of domain decomposition techniques, some theoretical foundations have been laid in $[3,4]$. Recent research activities concentrate on dual-primal finite element tearing and interconnecting (DP-FETI) methods (see $[62,63,65,71]$ ) and on such methods that use individual triangulations of the subdomains invoking nonmatching grids, also known as mortar edge element methods (cf., e.g., [11, 12,27,32,38-40,55-58,69]).

The main focus of this paper is on adaptive algorithms for the numerical solution of mortar edge element discretized eddy currents problems in three space dimensions. We note that the mortar element methodology in domain decomposition approaches, originally due to Bernardi, Maday, and Patera [13, 14], has been systematically developed, analyzed, and implemented for a variety of problems. The bulk of the work has been devoted to second order elliptic boundary value problems addressing both the issue of efficient multilevel iterative solvers and mesh 
adaptivity relying on appropriate a posteriori error estimators for the global discretization error (cf., e.g., [20, 21, 29, 30,41, 47,67]; see also the monograph [68] for further references).

The paper is organized as follows: In section 1 , we will provide a comprehensive introduction to the Hilbert space $H(\operatorname{curl} ; \Omega)$ and its trace spaces which play a central role in the construction of suitable Lagrange multipliers within the mortar edge element methodology. The following section 3 is devoted to the macro-hybrid variational formulation of the eddy currents equations with respect to a geometrically conforming, non overlapping decomposition of the computational domain [38]. The mortar edge element approximation, which is presented in section 4, tries to mimic the macro-hybrid variational formulation in the discrete regime. We focus on the proper construction of the Lagrange multiplier space and refer to some recently obtained optimal a priori estimates for the global discretization errors [69]. Section 5 deals with an efficient multilevel iterative solver for the solution of the saddle point problems arising from the mortar edge element approximations. Its main characteristics is a hybrid smoother that appropriately takes care of the non trivial kernel of the discrete curl operator. The smoother consists of two steps: the first step involves preconditioned Richardson-type iterative sweeps on the fully edge element discretized problems, whereas the second step is a defect correction on the subspace of irrotational vector fields. Particular emphasis is devoted to the development and analysis of an efficient and reliable residual-type a posteriori error estimator which is addressed in detail in section 6. By using a Helmholtz decomposition of the global discretization errors into its irrotational and weakly solenoidal parts, both the reliability and the local efficiency of the estimator are shown. Finally, the concluding section 7 is concerned with an illustration of the performance of the multilevel iterative solver and the residual-type a posteriori error estimator for an academic text example and two real-world applications. The first one is about the computation of parasitic inductivities in electric drives for high power electro motors which amounts to the solution of the eddy currents equations and constitutes the basis for an optimization of the operational behavior of such devices. The second example deals with the simulation of Logging-While-Drilling (LWD) tools in oil exploration which gives rise to the computation of electromagnetic fields satisfying the time-harmonic Maxwell equations. Although not covered by the theory presented in this paper, the simulation results indicate that the mortar approach can as well be used in the time-harmonic case.

\section{The Hilbert spaces $\mathbf{H}(\operatorname{curl} ; \Omega), \mathbf{H}(\operatorname{div} ; \Omega)$ and its trace spaces}

In the sequel, we assume $\Omega \subset \mathbb{R}^{3}$ to be a simply connected polyhedral domain with boundary $\Gamma=\partial \Omega$ which can be split into $n$ open faces $\Gamma_{i}, 1 \leq i \leq n$, such that $\Gamma=\cup_{i=1}^{n} \bar{\Gamma}_{i}$. We denote by $\mathcal{D}(\Omega)$ the space of all infinitely often differentiable functions with compact support in $\Omega$ and by $\mathcal{D}^{\prime}(\Omega)$ its dual space referring to $<\cdot, \cdot>$ as the dual pairing between $\mathcal{D}^{\prime}(\Omega)$ and $\mathcal{D}(\Omega)$. The paper adopts standard notation for Lebesgue and Sololvev spaces and norms.

We remind that the space $\mathbf{H}(\mathbf{c u r l} ; \Omega)$ is defined by

$$
\mathbf{H}(\operatorname{curl} ; \Omega):=\left\{\mathbf{q} \in \mathbf{L}^{2}(\Omega) \mid \operatorname{curl} \mathbf{q} \in \mathbf{L}^{2}(\Omega)\right\},
$$

where $\mathbf{L}^{2}(\Omega):=L^{2}(\Omega)^{3}$. It is a Hilbert space with respect to the inner product

$$
(\mathbf{j}, \mathbf{q})_{c u r l, \Omega}:=(\mathbf{j}, \mathbf{q})_{0, \Omega}+(\operatorname{curl} \mathbf{j}, \operatorname{curl} \mathbf{q})_{0, \Omega} \quad, \quad \mathbf{j}, \mathbf{q} \in H(\operatorname{curl} ; \Omega) .
$$


The associated norm will be denoted by $\|\cdot\|_{\text {curl }, \Omega}$.

We denote by $\mathbf{H}\left(\operatorname{curl}^{\mathbf{0}} ; \Omega\right)$ the subspace of irrotational vector fields

$$
\mathbf{H}\left(\operatorname{curl}^{\mathbf{0}} ; \Omega\right)=\{\mathbf{q} \in \mathbf{H}(\operatorname{curl} ; \Omega) \mid \operatorname{curl} \mathbf{q}=\mathbf{0}\} .
$$

It admits the characterization

$$
\mathbf{H}\left(\operatorname{curl}^{\mathbf{0}} ; \Omega\right)=\operatorname{grad} H^{1}(\Omega) .
$$

We further refer to $\mathbf{H}^{\perp}(\mathbf{c u r l} ; \Omega)$ as the orthogonal complement

$$
\mathbf{H}^{\perp}(\operatorname{curl} ; \Omega)=\left\{\mathbf{q} \in \mathbf{H}(\operatorname{curl} ; \Omega) \mid\left(\mathbf{q}, \mathbf{q}^{\mathbf{0}}\right)_{0, \Omega}=0, \mathbf{q}^{\mathbf{0}} \in \mathbf{H}\left(\operatorname{curl}^{\mathbf{0}} ; \Omega\right)\right\} .
$$

In view of (2.4), the subspace $\mathbf{H}^{\perp}(\mathbf{c u r l} ; \Omega)$ can be interpreted as the subspace of weakly solenoidal vector fields.

It follows readily from (2.3) and (2.5) that the Hilbert space $\mathbf{H}(\mathbf{c u r l} ; \Omega)$ admits the following Helmholtz decomposition

$$
\mathbf{H}(\operatorname{curl} ; \Omega)=\mathbf{H}\left(\operatorname{curl}^{\mathbf{0}} ; \Omega\right) \oplus \mathbf{H}^{\perp}(\operatorname{curl} ; \Omega) .
$$

Likewise, the space $\mathbf{H}(\operatorname{div} ; \Omega)$ is defined by

$$
\mathbf{H}(\operatorname{div} ; \Omega):=\left\{\mathbf{q} \in \mathbf{L}^{2}(\Omega) \mid \operatorname{div} \mathbf{q} \in L^{2}(\Omega)\right\},
$$

which is a Hilbert space with respect to the inner product

$$
(\mathbf{j}, \mathbf{q})_{\operatorname{div}, \Omega}:=(\mathbf{j}, \mathbf{q})_{0, \Omega}+(\operatorname{div} \mathbf{j}, \operatorname{div} \mathbf{q})_{0, \Omega} \quad, \quad \mathbf{j}, \mathbf{q} \in H(\operatorname{div} ; \Omega) .
$$

We will denote $\|\cdot\|_{d i v, \Omega}$ the associated norm.

We refer to $\mathbf{H}\left(\operatorname{div}^{0} ; \Omega\right)$ as the subspace of solenoidal vector fields

$$
\mathbf{H}\left(\operatorname{div}^{0} ; \Omega\right)=\{\mathbf{q} \in \mathbf{H}(\operatorname{div} ; \Omega) \mid \operatorname{div} \mathbf{q}=\mathbf{0}\},
$$

which can be characterized according to

$$
\mathbf{H}\left(\operatorname{div}^{0} ; \Omega\right)=\operatorname{curl} \mathbf{H}(\mathbf{c u r l} ; \Omega) .
$$

The orthogonal complement is denoted by

$$
\mathbf{H}^{\perp}(\operatorname{div} ; \Omega)=\left\{\mathbf{q} \in \mathbf{H}(\operatorname{div} ; \Omega) \mid\left(\mathbf{q}, \mathbf{q}^{\mathbf{0}}\right)_{0, \Omega}=0, \mathbf{q}^{\mathbf{0}} \in \mathbf{H}\left(\operatorname{div}^{0} ; \Omega\right)\right\} .
$$

Due to (2.10), it can be viewed as the subspace of weakly irrotational vector fields.

Consequently, with regard to (2.9) and (2.11), the Hilbert space $\mathbf{H}(\operatorname{div} ; \Omega)$ has the following Helmholtz decomposition

$$
\mathbf{H}(\operatorname{div} ; \Omega)=\mathbf{H}\left(\operatorname{div}^{0} ; \Omega\right) \oplus \mathbf{H}^{\perp}(\operatorname{div} ; \Omega) .
$$

We set $\mathcal{D}(\bar{\Omega}):=\left\{\left.\varphi\right|_{\Omega} \mid \varphi \in \mathcal{D}\left(\mathbb{R}^{3}\right)\right\}$. For vector fields $\mathbf{q} \in \mathcal{D}(\bar{\Omega})^{3}$ we consider the normal component trace mapping

$$
\left.\eta_{\mathbf{n}}(\mathbf{q})\right|_{\Gamma_{i}}:=\left.\mathbf{n}_{i} \cdot \mathbf{q}\right|_{\Gamma_{i}}, 1 \leq i \leq n,
$$

where $\mathbf{n}_{i}$ denotes the exterior unit normal vector on $\Gamma_{i}$. We recall that the space $\mathcal{D}(\bar{\Omega})^{3}$ is dense in $\mathbf{H}(\operatorname{div} ; \Omega)$ (cf., e.g., [33]; Thm. 2.1) and the normal component trace mapping $\eta_{\mathbf{n}}$ can be extended by continuity to a surjective, continuous linear mapping (cf. [33]; Thm. 2.2)

$$
\eta_{\mathbf{n}}: \mathbf{H}(\operatorname{div} ; \Omega) \rightarrow \mathbf{H}^{-1 / 2}(\Gamma) \text {. }
$$

We define $\mathbf{H}_{0}(\operatorname{div} ; \Omega)$ as the subspace of vector fields with vanishing normal components on $\Gamma$ :

$$
\mathbf{H}_{0}(\operatorname{div} ; \Omega):=\left\{\mathbf{q} \in \mathbf{H}(\operatorname{div} ; \Omega) \mid \eta_{\mathbf{n}}(\mathbf{q})=0\right\}
$$


In order to study the traces of vector fields $\mathbf{q} \in H(\operatorname{curl} ; \Omega)$, following [24-26], we introduce the spaces

$$
\begin{aligned}
\mathbf{L}_{\mathbf{t}}^{2}(\Gamma) & :=\left\{\mathbf{q} \in \mathbf{L}^{2}(\Omega) \mid \eta_{\mathbf{n}}(\mathbf{q})=0\right\} \\
\mathbf{H}_{-}^{1 / 2}(\Gamma) & :=\left\{\mathbf{q} \in \mathbf{L}_{\mathbf{t}}^{2}(\Gamma)|\mathbf{q}|_{\Gamma_{i}} \in \mathbf{H}^{1 / 2}(\Gamma), 1 \leq i \leq n\right\} .
\end{aligned}
$$

For $\mathbf{q} \in \mathcal{D}(\bar{\Omega})^{3}$ we define the tangential trace mapping

$$
\left.\gamma_{\mathbf{t}}\right|_{\Gamma_{i}}:=\left.\mathbf{q} \wedge \mathbf{n}_{i}\right|_{\Gamma_{i}}, 1 \leq i \leq n
$$

Further, we consider the tangential components trace mapping

$$
\left.\boldsymbol{\pi}_{\mathbf{t}}\right|_{\Gamma_{i}}:=\left.\mathbf{n}_{i} \wedge\left(\mathbf{q} \wedge \mathbf{n}_{i}\right)\right|_{\Gamma_{i}}, 1 \leq i \leq n
$$

Recalling that $\mathcal{D}(\bar{\Omega})^{3}$ is dense in $\mathbf{H}^{1}(\Omega)^{3}$, it is easy to see that the mappings $\gamma_{\mathbf{t}}$ and $\boldsymbol{\pi}_{\mathbf{t}}$ can be extended to linear continuous mappings from $\mathbf{H}^{1}(\Omega)$ into $\mathbf{H}_{-}^{1 / 2}(\Gamma)$.

However, the range of $\gamma_{\mathbf{t}}$ and the range of $\boldsymbol{\pi}_{\mathbf{t}}$ are proper subspaces of $\mathbf{H}_{-}^{1 / 2}(\Gamma)$ (cf., e.g., [24]; Thm. 1.5).

Assume $\Gamma_{i}, \Gamma_{j} \subset \Gamma, i \neq j$ such that $E_{i j}=\bar{\Gamma}_{i} \cap \bar{\Gamma}_{j} \neq \emptyset$, and let $\varphi_{i} \in H^{1 / 2}\left(\Gamma_{i}\right)$ and $\varphi_{j} \in H^{1 / 2}\left(\Gamma_{j}\right)$. We define equality on $E_{i j}$ by means of

$$
\varphi_{i}={ }_{E_{i j}} \varphi_{j} \Longleftrightarrow \int_{\Gamma_{i}} \int_{\Gamma_{j}} \frac{|\varphi(\mathbf{x})-\varphi(\mathbf{y})|^{2}}{|\mathbf{x}-\mathbf{y}|^{3}} d \sigma(\mathbf{x}) d \sigma(\mathbf{y})<\infty .
$$

We further introduce the set of indices

$$
\mathcal{I}_{i}:=\left\{j \in\{1, \ldots, N\} \mid \bar{\Gamma}_{i} \cap \bar{\Gamma}_{j}=E_{i j} \neq \emptyset\right\}
$$

and define the space

$$
\mathbf{H}_{\|}^{1 / 2}(\Gamma):=\left\{\mathbf{q} \in \mathbf{H}_{-}^{1 / 2}(\Gamma) \mid \mathbf{t}_{i j} \cdot \mathbf{q}_{i}={ }_{E_{i j}} \mathbf{t}_{i j} \cdot \mathbf{q}_{j}, 1 \leq i \leq N, j \in \mathcal{I}_{i}\right\},
$$

where $\mathbf{t}_{i j}$ is the unit vector parallel to $E_{i j}$.

The space $\mathbf{H}_{\|}^{1 / 2}(\Gamma)$ is a Hilbert space with respect to the norm

$$
\|\mathbf{q}\|_{\|, 1 / 2, \Gamma}:=\sum_{i=1}^{N}\left\|\mathbf{q}_{i}\right\|_{1 / 2, \Gamma_{i}}^{2}+\sum_{i=1}^{N} \sum_{j \in \mathcal{I}_{i}} \int_{\Gamma_{i}} \int_{\Gamma_{j}} \frac{\left|\mathbf{t}_{i j} \cdot \mathbf{q}_{i}(\mathbf{x})-\mathbf{t}_{i j} \cdot \mathbf{q}_{j}(\mathbf{y})\right|^{2}}{|\mathbf{x}-\mathbf{y}|^{3}} d \sigma(\mathbf{x}) d \sigma(\mathbf{y}) .
$$

The tangential components trace mapping

$$
\boldsymbol{\pi}_{\mathbf{t}}: \mathbf{H}^{1}(\Omega) \rightarrow \mathbf{H}_{\|}^{1 / 2}(\Gamma)
$$

is a continuous, surjective linear mapping.

In order to establish related mapping properties of the tangential trace mapping $\gamma_{\mathbf{t}}$ we introduce the space

$$
\mathbf{H}_{\perp}^{1 / 2}(\Gamma):=\left\{\mathbf{q} \in \mathbf{H}_{-}^{1 / 2}(\Gamma) \mid \mathbf{t}_{i} \cdot \mathbf{q}_{i}={ }_{e_{i j}} \mathbf{t}_{j} \cdot \mathbf{q}_{j}, 1 \leq i \leq N, j \in \mathcal{I}_{i}\right\}
$$

which is a Hilbert space with respect to the norm

$$
\|\mathbf{q}\|_{\perp, 1 / 2, \Gamma}:=\sum_{i=1}^{N}\left\|\mathbf{q}_{i}\right\|_{1 / 2, \Gamma_{i}}^{2}+\sum_{i=1}^{N} \sum_{j \in \mathcal{I}_{i}} \int_{\Gamma_{i}} \int_{\Gamma_{j}} \frac{\left|\mathbf{t}_{i} \cdot \mathbf{q}_{i}(\mathbf{x})-\mathbf{t}_{j} \cdot \mathbf{q}_{j}(\mathbf{y})\right|^{2}}{|\mathbf{x}-\mathbf{y}|^{3}} d \sigma(\mathbf{x}) d \sigma(\mathbf{y}) .
$$

The tangential trace mapping $\gamma_{\mathbf{t}}$

$$
\gamma_{\mathbf{t}}: \mathbf{H}^{1}(\Omega) \rightarrow \mathbf{H}_{\perp}^{1 / 2}(\Gamma)
$$

is a continuous, surjective linear mapping. 
In the sequel, we will refer to $\mathbf{H}_{\|}^{-1 / 2}(\Gamma)$ and $\mathbf{H}_{\perp}^{-1 / 2}(\Gamma)$ as the dual spaces of $\mathbf{H}_{\|}^{1 / 2}(\Gamma)$ and $\mathbf{H}_{\perp}^{1 / 2}(\Gamma)$ with $\mathbf{L}_{\mathbf{t}}^{2}(\Gamma)$ as the pivot space.

For a smooth function $u \in \mathcal{D}(\bar{\Omega})$ the tangential gradient operator $\nabla_{\Gamma}=\left.\operatorname{grad}\right|_{\Gamma}$ is defined as the tangential components trace of the gradient operator $\nabla$

$$
\left.\nabla_{\Gamma} u\right|_{\Gamma_{i}}:=\nabla_{\Gamma_{i}} u=\pi_{\mathbf{t}, i}(\nabla u)=\mathbf{n}_{i} \wedge\left(\nabla u \wedge \mathbf{n}_{i}\right), 1 \leq i \leq n .
$$

Since $\mathcal{D}(\bar{\Omega})$ is dense in $H^{2}(\Omega)$, we easily deduce that the tangential gradient operator is a continuous linear mapping

$$
\nabla_{\Gamma}: H^{3 / 2}(\Gamma) \rightarrow \mathbf{H}_{\|}^{1 / 2}(\Gamma) .
$$

The tangential divergence operator

$$
\left.\operatorname{div}\right|_{\tau}: \mathbf{H}_{\|}^{-1 / 2}(\Gamma) \rightarrow H^{-3 / 2}(\Gamma)
$$

is defined as the adjoint operator of $-\nabla_{\Gamma}$

$$
<\left.\operatorname{div}\right|_{\Gamma} \mathbf{q}, u>_{3 / 2, \Gamma}=-<\mathbf{q}, \nabla_{\Gamma} u>_{\|, 1 / 2, \Gamma} \quad, \quad u \in H^{3 / 2}(\Gamma), \mathbf{q} \in \mathbf{H}_{\|}^{-1 / 2}(\Gamma) .
$$

Finally, for $u \in \mathcal{D}(\Omega)$ we define the tangential curl operator curl $\left.\right|_{\tau}$ as the tangential trace of the gradient operator

$$
\left.\operatorname{curl}_{\tau} u\right|_{\Gamma_{i}}=\left.\operatorname{curl}\right|_{\Gamma_{i}} u=\gamma_{\mathbf{t}, i}(\nabla u)=\nabla u \wedge \mathbf{n}_{i}, 1 \leq i \leq n .
$$

The vectorial tangential curl operator is a linear continuous mapping

$$
\operatorname{curl}_{\tau}: H^{3 / 2}(\Gamma) \rightarrow \mathbf{H}_{\perp}^{1 / 2}(\Gamma) .
$$

The scalar tangential curl operator

$$
\operatorname{curl}_{\tau}: \mathbf{H}_{\perp}^{-1 / 2}(\Gamma) \rightarrow H^{-3 / 2}(\Gamma)
$$

is defined as the adjoint of the vectorial tangential curl operator $\left.\operatorname{curl}\right|_{\tau}$

$$
<\left.\operatorname{curl}\right|_{\tau} \mathbf{q}, u>_{3 / 2, \Gamma}=<\mathbf{q},\left.\operatorname{curl}\right|_{\Gamma} u>_{\perp, 1 / 2, \Gamma} \quad, \quad u \in H^{3 / 2}(\Gamma), \mathbf{q} \in \mathbf{H}_{\perp}^{-1 / 2}(\Gamma) .
$$

The range spaces of the tangential trace mapping $\gamma_{\mathbf{t}}$ and the tangential components trace mapping $\pi_{\mathbf{t}}$ on $H(\mathbf{c u r l} ; \Omega)$ can be characterized by means of the spaces

$$
\begin{aligned}
\mathbf{H}^{-1 / 2}\left(\left.\operatorname{div}\right|_{\Gamma}, \Gamma\right) & :=\left\{\boldsymbol{\lambda} \in \mathbf{H}_{\|}^{-1 / 2}(\Gamma)|\operatorname{div}|_{\Gamma} \boldsymbol{\lambda} \in H^{-1 / 2}(\Gamma)\right\}, \\
\mathbf{H}^{-1 / 2}\left(\left.\operatorname{curl}\right|_{\Gamma}, \Gamma\right) & :=\left\{\boldsymbol{\lambda} \in \mathbf{H}_{\perp}^{-1 / 2}(\Gamma)|\operatorname{curl}|_{\Gamma} \boldsymbol{\lambda} \in H^{-1 / 2}(\Gamma)\right\} .
\end{aligned}
$$

It can be shown that the tangential trace mapping is a continuous linear mapping

$$
\gamma_{\mathbf{t}}: \mathbf{H}(\operatorname{curl} ; \Omega) \rightarrow \mathbf{H}^{-1 / 2}\left(\left.\operatorname{div}\right|_{\Gamma}, \Gamma\right)
$$

whereas the tangential components trace mapping is a continuous linear mapping

$$
\boldsymbol{\pi}_{\mathbf{t}}: \mathbf{H}(\operatorname{curl} ; \Omega) \rightarrow \mathbf{H}^{-1 / 2}\left(\left.\operatorname{curl}\right|_{\Gamma}, \Gamma\right) .
$$

The previous results imply that the tangential divergence of the tangential trace and the scalar tangential curl of the tangential components trace coincide: For $\mathbf{j} \in \mathbf{H}(\mathbf{c u r l} ; \Omega)$ there holds

$$
\left.\operatorname{div}\right|_{\Gamma}(\mathbf{j} \wedge \mathbf{n})=\left.\operatorname{curl}\right|_{\Gamma}(\mathbf{n} \wedge(\mathbf{j} \wedge \mathbf{n}))=\mathbf{n} \cdot \operatorname{curl} \mathbf{j} .
$$

We define $\mathbf{H}_{\mathbf{0}}(\mathbf{c u r l} ; \Omega)$ as the subspace of $\mathbf{H}(\mathbf{c u r l} ; \Omega)$ with vanishing tangential trace components on $\Gamma$

$$
\mathbf{H}_{\mathbf{0}}(\mathbf{c u r l} ; \Omega):=\left\{\mathbf{q} \in \mathbf{H}(\operatorname{curl} ; \Omega) \mid \boldsymbol{\pi}_{\mathbf{t}}(\mathbf{q})=0\right\}
$$


and note that $\mathbf{H}_{\mathbf{0}}(\mathbf{c u r l} ; \Omega)$ admits the Helmholtz decomposition

$$
\mathbf{H}_{\mathbf{0}}(\operatorname{curl} ; \Omega)=\mathbf{H}_{\mathbf{0}}\left(\operatorname{curl}^{\mathbf{0}} ; \Omega\right) \oplus \mathbf{H}_{\mathbf{0}}^{\perp}(\operatorname{curl} ; \Omega),
$$

where $\mathbf{H}_{\mathbf{0}}\left(\operatorname{curl}^{\mathbf{0}} ; \Omega\right)=\operatorname{grad} H_{0}^{1}(\Omega)$ is the subspace of irrotational vector fields and $\mathbf{H}_{\mathbf{0}}^{\perp}(\mathbf{c u r l} ; \Omega)$ its orthogonal complement.

We also consider Helmholtz decompositions of the trace spaces, namely

$$
\mathbf{H}^{-1 / 2}\left(\operatorname{div}_{\tau} ; \Gamma\right)=\mathbf{H}^{-1 / 2}\left(\operatorname{div}_{\tau}^{0} ; \Gamma\right) \oplus \mathbf{H}^{-1 / 2, \perp}\left(\operatorname{div}_{\tau} ; \Gamma\right),
$$

where $\mathbf{H}^{-1 / 2}\left(\operatorname{div}_{\tau}^{0} ; \Gamma\right)$ is the subspace of solenoidal vector fields

$$
\mathbf{H}^{-1 / 2}\left(\operatorname{div}_{\tau}^{0} ; \Gamma\right):=\left\{\boldsymbol{\mu} \in \mathbf{H}^{-1 / 2}\left(\operatorname{div}_{\tau} ; \Gamma\right) \mid \operatorname{div}_{\tau} \boldsymbol{\mu}=0\right\}
$$

and $\mathbf{H}^{-1 / 2, \perp}\left(\operatorname{div}_{\tau} ; \Gamma\right)$ is its orthogonal complement

$$
\begin{aligned}
& \mathbf{H}^{-1 / 2, \perp}\left(\operatorname{div}_{\tau} ; \Gamma\right):= \\
& \left\{\boldsymbol{\mu} \in \mathbf{H}^{-1 / 2}\left(\operatorname{div}_{\tau} ; \Gamma\right) \mid<\boldsymbol{\mu}, \boldsymbol{\mu}^{\mathbf{0}}>=0, \boldsymbol{\mu}^{\mathbf{0}} \in \mathbf{H}^{-1 / 2}\left(\operatorname{div}_{\tau}^{0} ; \Gamma\right)\right\} .
\end{aligned}
$$

Likewise, we have

$$
\mathbf{H}^{-1 / 2}\left(\operatorname{curl}_{\tau} ; \Gamma\right)=\mathbf{H}^{-1 / 2}\left(\operatorname{curl}_{\tau}^{0} ; \Gamma\right) \oplus \mathbf{H}^{-1 / 2, \perp}\left(\operatorname{curl}_{\tau} ; \Gamma\right) .
$$

Here, $\mathbf{H}^{-1 / 2}\left(\operatorname{curl}_{\tau}^{0} ; \Gamma\right)$ stands for the subspace of irrotational vector fields

$$
\mathbf{H}^{-1 / 2}\left(\operatorname{curl}_{\tau}^{0} ; \Gamma\right):=\left\{\boldsymbol{\mu} \in \mathbf{H}^{-1 / 2}\left(\operatorname{curl}_{\tau} ; \Gamma\right) \mid \operatorname{curl}_{\tau} \boldsymbol{\mu}=0\right\}
$$

and $\mathbf{H}^{-1 / 2, \perp}\left(\operatorname{curl}_{\tau} ; \Gamma\right)$ refers to its orthogonal complement

$$
\begin{aligned}
& \mathbf{H}^{-1 / 2, \perp}\left(\operatorname{curl}_{\tau} ; \Gamma\right):= \\
& \left\{\boldsymbol{\mu} \in \mathbf{H}^{-1 / 2}\left(\operatorname{curl}_{\tau} ; \Gamma\right) \mid<\boldsymbol{\mu}, \boldsymbol{\mu}^{\mathbf{0}}>=0, \boldsymbol{\mu}^{\mathbf{0}} \in \mathbf{H}^{-1 / 2}\left(\operatorname{curl}_{\tau}^{0} ; \Gamma\right)\right\} .
\end{aligned}
$$

\section{Macrohybrid formulation of the eddy currents equation}

The quasistationary limit of Maxwell's equations, also known as the eddy currents equations, deals with currents caused by slowly in time varying electromagnetic fields. Since wave propagation can be neglected, Maxwell's equations reduce to a parabolic type initial-value problem. Assuming for simplicity homogeneous Dirichlet boundary conditions in terms of vanishing tangential components traces on $\partial \Omega$, its variational formulation amounts to the computation of $\mathbf{j} \in \mathbf{H}^{1}\left((0, T) ; \mathbf{L}^{2}(\Omega)\right) \cap \mathbf{L}^{2}\left((0, T) ; \mathbf{H}_{\mathbf{0}}(\mathbf{c u r l} ; \Omega)\right)$ such that $\left.\mathbf{j}\right|_{t=0}=\mathbf{j}_{\mathbf{0}}$ and

$\left.\int_{\Omega}\left(\boldsymbol{\sigma} \frac{\partial \mathbf{j}}{\partial t} \cdot \mathbf{q}+\boldsymbol{\mu}^{-\mathbf{1}} \operatorname{curl} \mathbf{j} \cdot \operatorname{curl} \mathbf{q}\right) d \mathbf{x}=\int_{\Omega} \mathbf{g} \cdot \mathbf{q} d x \quad, \quad \mathbf{q} \in \mathbf{H}_{\mathbf{0}}(\operatorname{curl} ; \Omega)\right)$.

Here, $\Omega$ stands for a bounded polyhedral domain in $\mathbb{R}^{3}$, whereas the data $\mathbf{j} \mathbf{0}, \mathbf{g}$ are supposed to satisfy $\mathbf{j}_{\mathbf{0}}, \mathbf{f} \in \mathbf{H}^{\mathbf{1}}(\Omega) \cap \mathbf{H}\left(\operatorname{div}^{0} ; \Omega\right)$. Moreover, the permeability $\boldsymbol{\mu}$ and the conductivity $\boldsymbol{\sigma}$ are assumed to be uniformly positive definite and uniformly positive semidefinite matrix-valued functions on $\Omega$. Implicit discretization in time by the backward Euler scheme gives rise to an elliptic boundary value for the double curl operator which will serve as a model problem throughout this paper. It can be stated as follows:

Find $\mathbf{j} \in \mathbf{H}_{\mathbf{0}}(\mathbf{c u r l} ; \Omega)$ such that

$$
a_{\Omega}(\mathbf{j}, \mathbf{q})=\ell(\mathbf{q}) \quad, \quad \mathbf{q} \in \mathbf{H}_{\mathbf{0}}(\operatorname{curl} ; \Omega),
$$


where the bilinear form $a_{\Omega}(\cdot, \cdot): \mathbf{H}_{\mathbf{0}}(\mathbf{c u r l} ; \Omega) \times \mathbf{H}_{\mathbf{0}}(\operatorname{curl} ; \Omega) \rightarrow \mathbb{R}$ and the functional $\ell(\cdot): \mathbf{H}_{\mathbf{0}}(\operatorname{curl} ; \Omega) \rightarrow \mathbb{R}$ are given by

$$
\begin{aligned}
a_{\Omega}(\mathbf{j}, \mathbf{q}) & :=\int_{\Omega}[\chi \operatorname{curl} \mathbf{j} \cdot \mathbf{c u r l} \mathbf{q}+\boldsymbol{\sigma} \mathbf{j} \cdot \mathbf{q}] d \mathbf{x}, \quad \mathbf{j}, \mathbf{q} \in \mathbf{H}_{\mathbf{0}}(\operatorname{curl} ; \Omega), \\
\ell(\mathbf{q}) & :=\int_{\Omega} \mathbf{f} \cdot \mathbf{q} d \mathbf{x}, \mathbf{q} \in \mathbf{H}_{\mathbf{0}}(\operatorname{curl} ; \Omega),
\end{aligned}
$$

where we have set $\chi=\boldsymbol{\mu}^{-\mathbf{1}}$. The bilinear form is not elliptic in case of non conducting subregions $\Omega_{n c} \subset \Omega$ where usually a gauge condition

$$
\int_{\Omega_{n c}} \varepsilon \mathbf{j} \cdot \mathbf{q} d \mathbf{x}=0 \quad, \quad \mathbf{q} \in \mathbf{H}_{\mathbf{0}}\left(\operatorname{curl}^{0} ; \Omega_{n c}\right)
$$

is imposed with $\varepsilon$ denoting the electric permittivity. However, for the sake of simplicity, we will assume $\Omega_{n c}=\emptyset$ such that $\sigma$ is uniformly positive definite on $\Omega$ and (3.1) admits a unique solution $\mathbf{j} \in \mathbf{H}_{\mathbf{0}}(\mathbf{c u r l} ; \Omega)$.

We consider the so-called macrohybrid formulation of (3.1) with respect to a non overlapping decomposition of the computational domain $\Omega$ into $N$ mutually disjoint subdomains

$$
\bar{\Omega}=\bigcup_{i=1}^{N} \bar{\Omega}_{i} \quad, \quad \Omega_{i} \cap \Omega_{j} \neq \emptyset \quad, \quad 1 \leq i \neq j \leq N .
$$

We assume the decomposition to be geometrically conforming, i.e., two adjacent subdomains either share a face, an edge, or a vertex. The skeleton $S$ of the decomposition

$$
S=\bigcup_{m=1}^{M} \bar{\gamma}_{m} \quad, \quad 1 \leq m \neq n \leq M,
$$

consists of the interfaces $\gamma_{m}, 1 \leq m \leq M$, between adjacent subdomains $\Omega_{i}$ and $\Omega_{j}$. We refer to $\gamma_{m(i)}$ as the mortar associated with subdomain $\Omega_{i}$, while the other face, which geometrically occupies the same place, is denoted by $\delta_{m(j)}$ and is called the nonmortar.

Based on (3.2) we introduce the product space

$$
\mathbf{X}:=\left\{\mathbf{q} \in \mathbf{L}^{\mathbf{2}}(\Omega)|\mathbf{q}|_{\Omega_{i}} \in \mathbf{H}^{\mathbf{1}}\left(\Omega_{i}\right) \cap \mathbf{H}\left(\mathbf{c u r l} ; \Omega_{i}\right),\left.(\mathbf{q} \wedge \mathbf{n})\right|_{\partial \Omega_{i} \cap \partial \Omega}=\mathbf{0}\right\}
$$

equipped with the norm

$$
\|\mathbf{q}\| \mathbf{X}:=\left(\sum_{i=1}^{N}\|\mathbf{q}\|_{\operatorname{curl}, \Omega_{i}^{2}}\right)^{\frac{1}{2}} .
$$

We further define

$$
\mathbf{V}:=\left\{\mathbf{q} \in \mathbf{X}|[\mathbf{q} \wedge \mathbf{n}]|_{\gamma_{m}} \in \mathbf{H}_{00}^{\frac{1}{2}}\left(\gamma_{m}\right), \gamma_{m} \subset S\right\}
$$

provided with the norm

$$
\|\mathbf{q}\|_{\mathbf{v}}:=\left(\|\mathbf{q}\|_{\mathbf{X}}^{2}+\|[\mathbf{q} \wedge \mathbf{n}]\|_{\frac{1}{2}, S}^{2}\right)^{\frac{1}{2}}
$$


where

$$
\|[\mathbf{q} \wedge \mathbf{n}]\|_{\frac{1}{2}, S}:=\left(\sum_{m=1}^{M}\|[\mathbf{q} \wedge \mathbf{n}]\|_{\mathbf{H}_{00}^{\frac{1}{2}}\left(\gamma_{m}\right)}^{2}\right)^{\frac{1}{2}}
$$

and

$$
\left.[\mathbf{q} \wedge \mathbf{n}]\right|_{\gamma_{m}}:=\left.(\mathbf{q} \wedge \mathbf{n})\right|_{\gamma_{m(i)}}-\left.(\mathbf{q} \wedge \mathbf{n})\right|_{\delta_{m(j)}} .
$$

LEMMA 3.1. There holds

$$
[\mathbf{q} \wedge \mathbf{n}]_{\gamma_{m}}=0,1 \leq m \leq M, \mathbf{q} \in \mathbf{V} \quad \Longrightarrow \quad \mathbf{V} \subset \mathbf{H}_{\mathbf{0}}(\operatorname{curl} ; \Omega) .
$$

PROOF. The proof is an easy consequence of Stokes' theorem.

In general, we cannot expect (3.10) to hold true. Therefore, we have to enforce some sort of weak continuity of the tangential traces across $\gamma_{m}$ by means of appropriately chosen Lagrange multipliers. A natural candidate for the multiplier space is

$$
\mathbf{M}(S):=\prod_{m=1}^{M} \mathbf{H}^{-\frac{1}{2}}\left(\operatorname{div}_{\tau} ; \gamma_{m}\right)
$$

equipped with the norm

$$
\|\boldsymbol{\mu}\|_{\mathbf{M}(S)}:=\left(\sum_{m=1}^{M}\left\|\left.\boldsymbol{\mu}\right|_{\gamma_{m}}\right\|_{-\frac{1}{2}, \gamma_{m}}^{2}\right)^{\frac{1}{2}} .
$$

We introduce the bilinear form $a(\cdot, \cdot): \mathbf{V} \times \mathbf{V} \rightarrow \mathbb{R}$ as the sum of the bilinear forms associated with the subdomain problems according to

$$
a(\mathbf{j}, \mathbf{q}):=\sum_{i=1}^{N} a_{\Omega_{i}}\left(\left.\mathbf{j}\right|_{\Omega_{i}},\left.\mathbf{q}\right|_{\Omega_{i}}\right)=\sum_{i=1}^{N} \int_{\Omega_{i}}[\chi \operatorname{curlj} \cdot \mathbf{c u r l} \mathbf{q}+\sigma \mathbf{j} \cdot \mathbf{q}] d \mathbf{x} .
$$

Furthermore, we define the bilinear form $b(\cdot, \cdot): \mathbf{V} \times \mathbf{M}(S) \rightarrow \mathbb{R}$ by means of

$$
b(\mathbf{q}, \boldsymbol{\mu}):=<\boldsymbol{\mu},[\mathbf{q} \wedge \mathbf{n}]>_{\frac{1}{2}, S},
$$

where

$$
<\cdot, \cdot>_{\frac{1}{2}, S}:=\sum_{m=1}^{M}<\cdot, \cdot>_{\frac{1}{2}, \gamma_{m}} .
$$

Then, the macro-hybrid variational formulation of (3.1) can be stated as follows:

Find $(\mathbf{j}, \boldsymbol{\lambda}) \in \mathbf{V} \times \mathbf{M}(S)$ such that

$$
\begin{array}{llll}
a(\mathbf{j}, \mathbf{q})+b(\mathbf{q}, \boldsymbol{\lambda}) & =\ell(\mathbf{q}), & \mathbf{q} \in \mathbf{V}, \\
b(\mathbf{j}, \boldsymbol{\mu}) & =0 \quad, \quad \boldsymbol{\mu} \in \mathbf{M}(S) .
\end{array}
$$

We denote by $B: \mathbf{V} \rightarrow \mathbf{M}(S)^{*}$ the operator associated with the bilinear form $b(\cdot, \cdot)$ according to

$$
<B \mathbf{q}, \boldsymbol{\mu}>_{\mathbf{M}(S)^{*}, \mathbf{M}(S)}=b(\mathbf{q}, \boldsymbol{\mu}) \quad, \quad \mathbf{q} \in \mathbf{V}, \boldsymbol{\mu} \in \mathbf{M}(S) .
$$

LEMma 3.2. The bilinear form $a(\cdot, \cdot)$ is elliptic on the kernel of B, i.e., there exists a positive constant $\alpha \in \mathbb{R}$ such that

$$
a(\mathbf{q}, \mathbf{q}) \geq \alpha\|\mathbf{q}\|_{\mathbf{V}}^{2} \quad, \quad \mathbf{q} \in \operatorname{Ker} B .
$$

ProOF. The assertion is an easy consequence of Ker $B=\mathbf{H}_{\mathbf{0}}(\mathbf{c u r l} ; \Omega)$. 
Lemma 3.3. (cf. [3,38] The bilinear form $b(\cdot, \cdot)$ satisfies the inf-sup condition

$$
\inf _{\boldsymbol{\mu} \in \mathbf{M}(S)} \sup _{\mathbf{q} \in \mathbf{V}} \frac{b(\mathbf{q}, \boldsymbol{\mu})}{\|\mathbf{q}\|_{\mathbf{V}}\|\boldsymbol{\mu}\|_{\mathbf{M}(S)}} \geq \beta>0 .
$$

Proof. Let $\boldsymbol{\mu} \in \mathbf{M}(S)$. For $\gamma_{m} \subset S, 1 \leq m \leq M$, choose $\mathbf{p}_{m} \in \mathbf{H}_{00}^{1 / 2}\left(\gamma_{m}\right)$ such that

$$
\left\|\mathbf{p}_{m}\right\|_{\mathbf{H}_{00}^{1 / 2}\left(\gamma_{m}\right)}=1 \quad \text { and } \quad<\mathbf{p}_{m}, \boldsymbol{\mu}>_{\frac{1}{2}, \gamma_{m}} \geq \frac{1}{2}\left\|\left.\boldsymbol{\mu}\right|_{\gamma_{m}}\right\|_{-\frac{1}{2}, \gamma_{m}} .
$$

We set $\mathbf{p}_{\partial \Omega_{i}}^{(j)}:=\tilde{\mathbf{p}}_{m} \in \mathbf{H}^{1 / 2}\left(\partial \Omega_{i}\right)$, where $\tilde{\mathbf{p}}_{m}$ is the extension by zero to the rest of $\partial \Omega_{i}$ with $\left.\mathbf{p}_{\partial \Omega_{i}}^{(j)}\right|_{\gamma_{m}} \wedge \mathbf{n}=\mathbf{p}_{m}$ and then determine $\mathbf{p}_{\Omega_{i}}^{(j)} \in \mathbf{H}\left(\mathbf{c u r l} ; \Omega_{i}\right)$ with $\left.\mathbf{p}_{\Omega_{i}}^{(j)}\right|_{\partial \Omega_{i}} \wedge$ $\mathbf{n}=\mathbf{p}_{\partial \Omega_{i}}^{(j)}$ and

$$
\left\|\mathbf{p}_{\Omega_{i}}^{(j)}\right\|_{c u r l, \Omega_{i}} \leq C\left\|\mathbf{p}_{\partial \Omega_{i}}^{(j)}\right\|_{+\frac{1}{2}, \gamma_{m}} \leq C\left\|\mathbf{p}_{\partial \Omega_{i}}^{(j)}\right\|_{\mathbf{H}_{00}^{1 / 2}\left(\gamma_{m}\right)}=C .
$$

We define $\mathbf{p} \in \mathbf{V}$ by $\left.\mathbf{p}\right|_{\Omega_{i}}:=\sum_{\gamma_{m} \subset \bar{\Omega}_{i}} \mathbf{p}_{\Omega_{i}}^{(j)}$. Observing (3.20) and (3.21), we have

$$
\begin{aligned}
& \sup _{\mathbf{q} \in \mathbf{V}} \frac{b(\mathbf{q}, \boldsymbol{\mu})}{\|\mathbf{q}\|_{\mathbf{V}}}\|\mathbf{p}\|_{\mathbf{V}} \geq c b(\mathbf{p}, \boldsymbol{\mu}) \\
& =c \sum_{m=1}^{M}<\mathbf{p}_{\gamma_{m(i)}}+\mathbf{p}_{\delta_{m(j)}},\left.\boldsymbol{\mu}\right|_{\gamma_{m}}>_{\frac{1}{2}, \gamma_{m}} \geq c \sum_{m=1}^{M}\left\|\left.\boldsymbol{\mu}\right|_{\gamma_{m}}\right\|_{-\frac{1}{2}, \gamma_{m}} \geq c\|\boldsymbol{\mu}\|_{\mathbf{M}(S)}
\end{aligned}
$$

which gives the assertion. Note that here and in the sequel $c$ and $C$ denote generic positive constants which are not necessarily the same at each occurrence.

As an immediate consequence of Lemma 3.2 and Lemma 3.3 we obtain the following existence and uniqueness result.

THEOREM 3.1. The macro-hybrid variational formulation (3.16) admits a unique solution $(\mathbf{j}, \boldsymbol{\lambda}) \in \mathbf{V} \times \mathbf{M}(S)$.

ProOF. The assertion is a classical result in the theory of saddle point problems (cf., e.g., [23]).

We note that the space $\mathbf{V}$ admits the Helmholtz decomposition

$$
\mathbf{V}=\mathbf{V}^{\mathbf{0}} \oplus \mathbf{V}^{\perp}
$$

where $\mathbf{V}^{\mathbf{0}}$ stands for the subspace of subdomainwise irrotational vector fields

$$
\mathbf{V}^{\mathbf{0}}:=\left\{\mathbf{q} \in \mathbf{V}|\operatorname{curl} \mathbf{q}|_{\Omega_{i}}=\mathbf{0}, 1 \leq i \leq N\right\}
$$

and $\mathbf{V}^{\perp}$ refers to its orthogonal complement

$$
\mathbf{V}^{\perp}:=\left\{\mathbf{q} \in \mathbf{V} \mid\left(\left.\mathbf{q}\right|_{\Omega_{i}},\left.\mathbf{q}^{\mathbf{0}}\right|_{\Omega_{i}}\right)_{0, \Omega_{i}}=0,1 \leq i \leq N, \mathbf{q}^{\mathbf{0}} \in \mathbf{V}^{\mathbf{0}}\right\} .
$$

The Helmholtz decomposition (2.34) of the tangential components trace spaces induces an associated decomposition of the multiplier space $\mathbf{M}(S)$ according to

$$
\mathbf{M}(S)=\mathbf{M}^{0}(S) \oplus \mathbf{M}^{\perp}(S),
$$

where $\mathbf{M}^{\mathbf{0}}(S)$ and $\mathbf{M}^{\perp}(S)$ are given by

$$
\begin{aligned}
\mathbf{M}^{\mathbf{0}}(S) & :=\left\{\boldsymbol{\mu} \in \mathbf{M}(S)\left|\operatorname{div}_{\tau} \boldsymbol{\mu}\right|_{\gamma_{m}}=0, \gamma_{m} \subset S\right\} \\
\mathbf{M}^{\perp}(S) & :=\left\{\boldsymbol{\mu} \in \mathbf{M}(S) \mid<\boldsymbol{\mu}, \boldsymbol{\mu}^{\mathbf{0}}>=0, \boldsymbol{\mu}^{\mathbf{0}} \in \mathbf{M}^{\mathbf{0}}(S)\right\} .
\end{aligned}
$$


Consequently, the solution $(\mathbf{j}, \boldsymbol{\lambda}) \in \mathbf{V} \times \mathbf{M}(S)$ can be split according to

$$
\begin{aligned}
\mathbf{j}=\mathbf{j}^{\mathbf{0}}+\mathbf{j}^{\perp} & , \quad \mathbf{j}^{\mathbf{0}} \in \mathbf{V}^{\mathbf{0}}, \mathbf{j}^{\perp} \in \mathbf{V}^{\perp}, \\
\boldsymbol{\lambda}=\boldsymbol{\lambda}^{\mathbf{0}}+\boldsymbol{\lambda}^{\perp} & , \quad \boldsymbol{\lambda}^{\mathbf{0}} \in \mathbf{M}^{\mathbf{0}}(S), \boldsymbol{\lambda}^{\perp} \in \mathbf{M}^{\perp}(S) .
\end{aligned}
$$

Since $\boldsymbol{\gamma}_{\mathbf{t}}\left(\mathbf{V}^{\mathbf{0}}\right) \subset \mathbf{M}^{\mathbf{0}}(S)$ and $\boldsymbol{\gamma}_{\mathbf{t}}\left(\mathbf{V}^{\perp}\right) \subset \mathbf{M}^{\perp}(S)$, it follows that the pair $\left(\mathbf{j}^{\mathbf{0}}, \boldsymbol{\lambda}^{\mathbf{0}}\right) \in$ $\mathbf{V}^{\mathbf{0}} \times \mathbf{M}^{\mathbf{0}}(S)$ satisfies the saddle point problem

$$
\begin{aligned}
& a\left(\mathbf{j}^{\mathbf{0}}, \mathbf{q}^{\mathbf{0}}\right)+b\left(\mathbf{q}^{\mathbf{0}}, \boldsymbol{\lambda}^{\mathbf{0}}\right)=\ell\left(\mathbf{q}^{\mathbf{0}}\right), \quad \mathbf{q}^{\mathbf{0}} \in \mathbf{V}^{\mathbf{0}}, \\
& b\left(\mathbf{j}^{\mathbf{0}}, \boldsymbol{\mu}^{\mathbf{0}}\right) \quad=\quad 0 \quad, \quad \boldsymbol{\mu}^{\mathbf{0}} \in \mathbf{M}^{\mathbf{0}}(S) \text {, }
\end{aligned}
$$

whereas the pair $\left(\mathbf{j}^{\perp}, \boldsymbol{\lambda}^{\perp}\right) \in \mathbf{V}^{\perp} \times \mathbf{M}^{\perp}(S)$ satisfies

$$
\begin{array}{llcll}
a\left(\mathbf{j}^{\perp}, \mathbf{q}^{\perp}\right)+b\left(\mathbf{q}^{\perp}, \boldsymbol{\lambda}^{\perp}\right) & = & \ell\left(\mathbf{q}^{\perp}\right) & , & \mathbf{q}^{\perp} \in \mathbf{V}^{\perp}, \\
b\left(\mathbf{j}^{\perp}, \boldsymbol{\mu}^{\perp}\right) & = & 0 & , & \boldsymbol{\mu}^{\perp} \in \mathbf{M}^{\perp}(S) .
\end{array}
$$

We remark that for computational purposes, the spaces $\mathbf{M}^{\mathbf{0}}(S)$ and $\mathbf{M}^{\perp}(S)$ are not easily accessible. Therefore, we consider instead the macro-hybrid formulation of the equation satisfied by $u \in H_{0}^{1}(\Omega)$ in the irrotational part $\mathbf{j}^{\mathbf{0}}=\operatorname{grad} u$ of the solution, which is as follows

$$
c_{\Omega}(u, v)=r(v) \quad, \quad v \in H_{0}^{1}(\Omega) .
$$

Here, the bilinear form $c_{\Omega}(\cdot, \cdot): H_{0}^{1}(\Omega) \times H_{0}^{1}(\Omega) \rightarrow \mathbb{R}$ and the functional $r$ : $H_{0}^{1}(\Omega) \rightarrow \mathbb{R}$ are given by

$$
\begin{aligned}
c_{\Omega}(u, v) & :=\int_{\Omega} \boldsymbol{\sigma} \operatorname{grad} u \cdot \operatorname{grad} v d \mathbf{x} \quad, \quad u, v \in H_{0}^{1}(\Omega), \\
r(v) & :=\ell(\operatorname{grad} v)-a_{\Omega}(\mathbf{j}, \operatorname{grad} v), \quad v \in H_{0}^{1}(\Omega) .
\end{aligned}
$$

To this end, we introduce the product space

$$
\begin{aligned}
X & :=\left\{v \in L^{\mathbf{2}}(\Omega)|v|_{\Omega_{i}} \in H^{\mathbf{1}}\left(\Omega_{i}\right),\left.v\right|_{\partial \Omega_{i} \cap \partial \Omega}=0\right\}, \\
\|v\|_{X} & :=\left(\sum_{i=1}^{N}\|v\|_{1, \Omega_{i}^{2}}\right)^{\frac{1}{2}}
\end{aligned}
$$

and its subspace

$$
\begin{aligned}
V & :=\left\{v \in X|[v]|_{\gamma_{m}} \in H_{00}^{\frac{1}{2}}\left(\gamma_{m}, \gamma_{m} \subset S\right\},\right. \\
\|v\|_{V} & :=\left(\|v\|_{X}^{2}+\|[v]\|_{\frac{1}{2}, S}^{2}\right)^{\frac{1}{2}}, \\
\|[v]\|_{\frac{1}{2}, S} & :=\left(\sum_{m=1}^{M}\|[v]\|_{H_{00}^{\frac{1}{2}\left(\gamma_{m}\right)}}^{2}\right)^{\frac{1}{2}}
\end{aligned}
$$

In order to enforce weak continuity of the traces across $\gamma_{m}$, we define the multiplier space

$$
\begin{aligned}
M(S) & :=\prod_{m=1}^{M} H^{-\frac{1}{2}}\left(\gamma_{m}\right), \\
\|\mu\|_{M(S)} & :=\left(\sum_{m=1}^{M}\left\|\left.\mu\right|_{\gamma_{m}}\right\|_{-\frac{1}{2}, \gamma_{m}}^{2}\right)^{\frac{1}{2}} .
\end{aligned}
$$


Moreover, we introduce the bilinear forms $c(\cdot, \cdot): V \times V \rightarrow \mathbb{R}$

$$
\left.c(u, v):=\sum_{i=1}^{N} c_{\Omega_{i}}\left(\left.u\right|_{\Omega_{i}},\left.v\right|_{\Omega_{i}}\right)=\sum_{i=1}^{N} \int_{\Omega_{i}} \sigma \operatorname{grad} u \cdot \operatorname{grad} v\right] d \mathbf{x}
$$

and $d(\cdot, \cdot): V \times M(S) \rightarrow \mathbb{R}$

$$
d(v, \mu):=-<\mu,[v]>_{\frac{1}{2}, S}
$$

The macro-hybrid variational formulation of (3.32) reads as follows:

Find $(u, \lambda) \in V \times M(S)$ such that

$$
\begin{array}{lll}
c(u, v)+d(v, \lambda) & =r(v), \quad v \in V, \\
d(u, \mu) & =0 \quad, \quad \mu \in M(S) .
\end{array}
$$

We refer to $D: V \rightarrow M(S)^{*}$ as the operator associated with the bilinear form $d(\cdot, \cdot)$ according to

$$
<D v, \mu>_{M(S)^{*}, M(S)}=d(v, \mu) \quad, \quad v \in V, \mu \in M(S) .
$$

LEMmA 3.4. The bilinear form $c(\cdot, \cdot)$ is elliptic on the kernel of $D$, i.e., there exists a positive constant $\alpha \in \mathbb{R}$ such that

$$
c(v, v) \geq \alpha\|v\|_{V}^{2} \quad, \quad v \in \operatorname{Ker} D
$$

Moreover, the bilinear form $d(\cdot, \cdot)$ satisfies the inf-sup condition

$$
\inf _{\mu \in M(S)} \sup _{v \in V} \frac{d(v, \mu)}{\|v\|_{V}\|\mu\|_{M(S)}} \geq \beta>0 .
$$

Proof. We refer to [20].

The previous Lemma 3.4 immediately gives rise to the following existence and uniqueness result.

THEOREM 3.2. The macro-hybrid variational formulation (3.42) admits a unique solution $(u, \lambda) \in V \times M(S)$.

\section{Mortar edge element approximations}

We consider individual simplicial triangulations $\mathcal{T}_{i}$ of the subdomains $\Omega_{i}, 1 \leq$ $i \leq N$, regardless the situation on the skeleton $S$ of the decomposition. In particular, the interfaces $\gamma_{m} \subset S, 1 \leq m \leq M$, inherit two different triangulations, namely the triangulation $\mathcal{T}_{\gamma_{m(i)}}$ of the mortar inherited from the triangulation $\mathcal{T}_{i}$ of $\Omega_{i}$ and the triangulation $\mathcal{T}_{\delta_{m(j)}}$ of the nonmortar inherited from the triangulation $\mathcal{T}_{j}$ of $\Omega_{j}$. Note that in general $\mathcal{T}_{\gamma_{m(i)}}$ and $\mathcal{T}_{\delta_{m(j)}}$ do not match on $\gamma_{m}$ due to the occurrence of nonconforming nodal points.

For $T \in \mathcal{T}_{i}, 1 \leq i \leq N$, and $T \in \mathcal{T}_{\gamma_{m(i)}}$ resp. $T \in \mathcal{T}_{\delta_{m(j)}}$ we denote by $h_{T}$ its diameter and by $\rho_{T}$ the radius of the largest ball resp. circle that can be inscribed to $T$. We assume that each of the triangulations $\mathcal{T}_{i}, 1 \leq i \leq N$, is shape regular in the sense that there exist constants $\kappa_{i}, 1 \leq i \leq n$, such that

$$
\frac{h_{T}}{\rho_{T}} \leq \kappa_{i} \text { uniformly for all } T \in \mathcal{T}_{i}
$$

and locally quasiuniform in the sense that there exist constants $0<\underline{\tau}_{i} \leq \bar{\tau}_{i}, 1 \leq$ $i \leq N$, such that

$$
0<\underline{\tau}_{i} h_{T}^{\prime} \leq h_{T} \leq \bar{\tau}_{i} h\left(T^{\prime}\right) \text { for all } T, T^{\prime} \in \mathcal{T}_{i} \text { with } T \cap T^{\prime} \neq \emptyset .
$$


These properties are inherited by the triangulations $\mathcal{T}_{\gamma_{m(i)}}$ and $\mathcal{T}_{\delta_{m(j)}}$.

We denote by $h_{i}$ and $h_{\gamma_{m(i)}}, h_{\delta_{m(j)}}$ global mesh sizes according to

$$
h_{i}:=\max \left\{\operatorname{diam} T \mid T \in \mathcal{T}_{i}\right\} \quad, \quad h_{\gamma_{m(i)}}:=\max \left\{\operatorname{diam} T \mid T \in \mathcal{T}_{\gamma_{m(i)}}\right\}
$$

and $h_{\delta_{m(j)}}$ defined analogously. Further, for $\Sigma_{i} \subset \bar{\Omega}_{i}$ we define $\mathcal{N}_{h}\left(\Sigma_{i}\right), \mathcal{F}_{h}\left(\Sigma_{i}\right)$, and $\mathcal{E}_{h}\left(\Sigma_{i}\right)$ as the sets of vertices, faces and edges of $\mathcal{T}_{i}$ in $\Sigma_{i}$. Likewise, for $\Sigma_{\gamma_{m(i)}} \subset$ $\gamma_{m(i)}, \gamma_{m(i)} \subset S$ and $\Sigma_{\delta_{m(j)}} \subset \delta_{m(j)}, \delta_{m(j)} \subset S$, we refer to $\mathcal{E}_{h}\left(\Sigma_{\gamma_{m(i)}}\right)$ and $\mathcal{E}_{h}\left(\Sigma_{\delta_{m(j)}}\right)$ as the set of edges of $\mathcal{T}_{\gamma_{m(i)}}$ and $\mathcal{T}_{\delta_{m(j)}}$ in $\Sigma_{\gamma_{m(i)}}$ and $\Sigma_{\delta_{m(j)}}$.

We assume that the granularities of the triangulations $\mathcal{T}_{\gamma_{m(i)}}$ and $\mathcal{T}_{\delta_{m(j)}}$ are such that there exist constants $0<\underline{\kappa}_{\gamma_{m}} \leq \bar{\kappa}_{\gamma_{m}}$ independent of $h_{\gamma_{m(i)}}$ and $h_{\delta_{m(j)}}$ satisfying

$$
\underline{\kappa}_{\gamma_{m}} h_{\gamma_{m(i)}} \leq h_{\delta_{m(j)}} \leq \bar{\kappa}_{\gamma_{m}} h_{\gamma_{m(i)}} \quad, \quad \gamma_{m} \subset S .
$$

For the discretization of

$$
\mathbf{H}_{0, \partial \Omega_{i} \cap \partial \Omega}\left(\mathbf{c u r l} ; \Omega_{i}\right):=\left\{\mathbf{q} \in \mathbf{H}\left(\mathbf{c u r l} ; \Omega_{i}\right)|\mathbf{q} \wedge \mathbf{n}|_{\partial \Omega_{i} \cap \partial \Omega}=0\right\}
$$

we will use Nédélec's curl-conforming edge elements as described in [49]. We recall that for a tetrahedron $T \in \mathcal{T}_{i}$ the edge element $\mathbf{N d}_{\mathbf{1}}(T)$ is defined by means of

$$
\mathbf{N d}_{\mathbf{1}}(T):=\left\{\mathbf{q}:=\mathbf{a}+\mathbf{b} \wedge \mathbf{x} \mid \mathbf{a}, \mathbf{b} \in \mathbf{R}^{\mathbf{3}}, \mathbf{x} \in T\right\}
$$

with the degrees of freedom given by the zero order moments of the tangential components with respect to the edges $E \in \mathcal{E}_{h}(T)$

$$
\ell_{E}(\mathbf{q}):=\int_{E} \mathbf{t}_{E} \cdot \mathbf{q} d s \quad, \quad E \in \mathcal{E}_{h}(T),
$$

where $\mathbf{t}_{E}$ stands for the tangential unit vector on $E$.

The edge element spaces $\mathbf{N d}_{\mathbf{1}}\left(\Omega_{i} ; \mathcal{T}_{i}\right)$ are then given as follows

$$
\mathbf{N d}_{\mathbf{1}}\left(\Omega_{i} ; \mathcal{T}_{i}\right):=\left\{\mathbf{q}_{\mathbf{h}} \in \mathbf{L}^{2}\left(\Omega_{i}\right)\left|\mathbf{q}_{\mathbf{h}}\right|_{T} \in \mathbf{N d}_{\mathbf{1}}(T), T \in \mathcal{T}_{i}\right\} .
$$

We refer to $\mathbf{N d}_{\mathbf{1}, \Gamma}\left(\Omega_{i} ; \mathcal{T}_{i}\right)$ as its subspaces of vector fields with vanishing tangential trace components on $\Gamma \cap \partial \Omega_{i}$, i.e.,

$$
\mathbf{N d}_{\mathbf{1}, \Gamma}\left(\Omega_{i} ; \mathcal{T}_{i}\right):=\left\{\mathbf{q}_{\mathbf{h}} \in \mathbf{N d}_{\mathbf{1}}\left(\Omega_{i} ; \mathcal{T}_{i}\right)\left|\boldsymbol{\pi}_{\mathbf{t}}(\mathbf{q})\right|_{\Gamma}=\mathbf{0}\right\} .
$$

For the proper specification of the Lagrange multiplier space in the discrete regime, we have to provide appropriate discretizations of the tangential components traces by means of Raviart-Thomas finite elements (cf., e.g., [23]). For a triangle $T \in$ $\mathcal{I}_{\delta_{m(j)}}, \delta_{m(j)} \subset S$, the lowest order Raviart-Thomas element $\mathbf{R T}_{\mathbf{0}}(T)$ is given by

$$
\mathbf{R T}_{\mathbf{0}}(T):=\left\{\mathbf{q}=\mathbf{a}+b \mathbf{x} \mid \mathbf{a} \in \mathbb{R}^{2}, b \in \mathbb{R}, \mathbf{x} \in T\right\}
$$

with the degrees of freedom given by the zero order moments of the normal components with respect to the edges $E \in \mathcal{E}_{h}(T)$

$$
\ell_{E}(\mathbf{q}):=\int_{E} \mathbf{n}_{E} \cdot \mathbf{q} d s \quad, \quad E \in \mathcal{E}_{h}(T),
$$

where $\mathbf{n}_{E}$ stands for the unit outer normal on $E$.

The Raviart-Thomas spaces $\mathbf{R T}_{\mathbf{0}}\left(\delta_{m(j)} ; \mathcal{I}_{\delta_{m(j)}}\right)$ are then given by

$$
\mathbf{R T}_{\mathbf{0}}\left(\delta_{m(j)} ; \mathcal{T}_{\delta_{m(j)}}\right):=\left\{\mathbf{q}_{\mathbf{h}} \in \mathbf{L}^{2}\left(\delta_{m(j)}\right)\left|\mathbf{q}_{\mathbf{h}}\right|_{T} \in \mathbf{R T}_{\mathbf{0}}(T), T \in \mathcal{T}_{\delta_{m(j)}}\right\} .
$$


We refer to $\mathbf{R T}_{\mathbf{0 , 0}}\left(\delta_{m(j)} ; \mathcal{T}_{\delta_{m(j)}}\right)$ as the subspaces of vector fields with vanishing normal components on $\delta_{m(j)}$, i.e.,

$$
\mathbf{R T}_{\mathbf{0 , 0}}\left(\delta_{m(j)} ; \mathcal{T}_{\delta_{m(j)}}\right):=\left\{\mathbf{q}_{\mathbf{h}} \in \mathbf{R} \mathbf{T}_{\mathbf{0}}\left(\delta_{m(j)} ; \mathcal{T}_{\delta_{m(j)}}\right)\left|\eta_{\mathbf{n}}\left(\mathbf{q}_{\mathbf{h}}\right)\right|_{\partial \delta_{m(j)}}=0\right\} .
$$

Based on these definitions we consider the product space

$$
\mathbf{V}_{\mathbf{h}}:=\left\{\mathbf{q}_{\mathbf{h}} \in \mathbf{L}^{2}(\Omega)\left|\mathbf{q}_{\mathbf{h}}\right|_{\Omega_{i}} \in \mathbf{N d}_{\mathbf{1}, \boldsymbol{\Gamma}}\left(\Omega_{i} ; \mathcal{T}_{i}\right), 1 \leq i \leq N\right\}
$$

equipped with the norm

$$
\left\|\mathbf{q}_{\mathbf{h}}\right\|_{\mathbf{V}_{\mathbf{h}}}:=\left(\left\|\mathbf{q}_{\mathbf{h}}\right\|_{\mathbf{X}}^{2}+\left\|\left.\left[\mathbf{q}_{\mathbf{h}} \wedge \mathbf{n}\right]\right|_{S}\right\|_{+\frac{1}{2}, h, S}^{2}\right)^{1 / 2}, \quad \mathbf{q}_{\mathbf{h}} \in \mathbf{V}_{\mathbf{h}},
$$

where $\|\cdot\|_{+\frac{1}{2}, h, S}$ is given by

$$
\left\|\left.\left[\mathbf{q}_{\mathbf{h}} \wedge \mathbf{n}\right]\right|_{S}\right\|_{+\frac{1}{2}, h, S}:=\left(\sum_{m=1}^{M}\left\|\left.\left[\mathbf{q}_{\mathbf{h}} \wedge \mathbf{n}\right]\right|_{\gamma_{m}}\right\|_{+\frac{1}{2}, h, \gamma_{m}}\right)^{1 / 2}
$$

and $\|\cdot\|_{+\frac{1}{2}, h, \gamma_{m}}$ stands for the mesh-dependent norm

$$
\left\|\left.\left[\mathbf{q}_{\mathbf{h}} \wedge \mathbf{n}\right]\right|_{\gamma_{m}}\right\|_{+\frac{1}{2}, h, \gamma_{m}}:=h^{-1 / 2}\left\|\left.\left[\mathbf{q}_{\mathbf{h}} \wedge \mathbf{n}\right]\right|_{\gamma_{m}}\right\|_{0, \gamma_{m}} .
$$

Due to the occurrence of nonconforming edges on the interfaces between adjacent subdomains, there is a lack of continuity across the interfaces: neither the tangential traces $\mathbf{q}_{\mathbf{h}} \wedge \mathbf{n}$ nor the tangential trace components $\mathbf{n} \wedge\left(\mathbf{q}_{\mathbf{h}} \wedge \mathbf{n}\right)$ can be

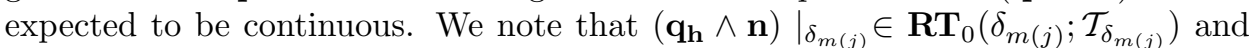
$\left.\left(\mathbf{n} \wedge\left(\mathbf{q}_{\mathbf{h}} \wedge \mathbf{n}\right)\right)\right|_{\delta_{m(j)}} \in \mathbf{N d}_{1}\left(\delta_{m(j)} ; \mathcal{T}_{\delta_{m(j)}}\right)$. Therefore, continuity can be enforced either in terms of the tangential traces or the tangential trace components. If we choose the tangential traces, the multiplier $\operatorname{space} \mathbf{M}_{\mathbf{h}}(S)$ can be constructed according to

$$
\mathbf{M}_{\mathbf{h}}(S):=\prod_{m=1}^{M} \mathbf{M}_{\mathbf{h}}\left(\delta_{m(j)}\right)
$$

with $\mathbf{M}_{\mathbf{h}}\left(\delta_{m(j)}\right)$ chosen such that

$$
\begin{aligned}
& \mathbf{R T}_{0,0}\left(\delta_{m(j)} ; \mathcal{T}_{\delta_{m(j)}}\right) \subset \mathbf{M}_{\mathbf{h}}\left(\delta_{m(j)}\right) \\
& \operatorname{dim} \mathbf{M}_{\mathbf{h}}\left(\delta_{m(j)}\right)=\operatorname{dim} \mathbf{R T}_{0,0}\left(\delta_{m(j)} ; \delta_{m(j)}\right) .
\end{aligned}
$$

The definition of $\mathbf{M}_{\mathbf{h}}\left(\delta_{m(j)}\right)$ has to be done in such way that $\mathbf{M}_{\mathbf{h}}\left(\delta_{m(j)}\right)$ contains constant vectors. This requires a subtle specification of the basis fields of $\mathbf{R T}_{0}\left(\delta_{m(j)} ; \mathcal{T}_{\delta_{m(j)}}\right)$. We refer to $\mathbf{q}_{E}$ as the basis field associated with an edge $E \in \mathcal{E}_{h}\left(\bar{\delta}_{m(j)}\right)$ according to

$$
\int_{E^{\prime}} \mathbf{n}_{E^{\prime}} \cdot \mathbf{q}_{E} d s=h_{\delta_{m(j)}} \delta_{E, E^{\prime}} \quad, \quad E^{\prime} \in \mathcal{E}_{h}\left(\bar{\delta}_{m(j)}\right) .
$$

We define $\mathbf{M}_{\mathbf{h}}\left(\delta_{m(j)}\right)$ by an extension of the basis field $\mathbf{q}_{E} \in \mathbf{T} \mathbf{R}_{0,0}\left(\delta_{m(j)} ; \mathcal{T}_{\delta_{m(j)}}\right)$ with respect to those edges $E \in \delta_{m(j)}$ that have at least one neighboring edge on the boundary $\partial \delta_{m(j)}$.

For this purpose, if $E \in \mathcal{E}_{h}\left(\delta_{m(j)}\right)$ is an interior edge, we denote by

$$
\mathcal{E}_{h}^{\partial \delta_{m(j)}}(E):=\left\{E^{\prime} \in \mathcal{E}_{h}\left(\partial \delta_{m(j)}\right) \mid E^{\prime} \subset \operatorname{supp} \mathbf{q}_{E}\right\},
$$

the set of the neighboring edges on $\partial \delta_{m(j)}$. 
On the other hand, if $E \in \mathcal{E}_{h}\left(\partial \delta_{m(j)}\right)$ is a boundary edge, we refer to

$$
\mathcal{E}_{h}^{\delta_{m(j)}}(E):=\left\{E^{\prime} \in \mathcal{E}_{h}\left(\delta_{m(j)}\right) \mid E^{\prime} \subset \operatorname{supp} \mathbf{q}_{E}\right\}
$$

as the set of neighboring edges in the interior of $\delta_{m(j)}$.

Finally we define

$$
\mathcal{E}_{h}^{\delta_{m(j)}}\left(\partial \delta_{m(j)}\right):=\bigcup_{E^{\prime} \in \mathcal{E}_{h}\left(\partial \delta_{m(j)}\right)} \mathcal{E}_{h}^{\delta_{m(j)}}\left(E^{\prime}\right)
$$

as the set of interior edges with a neighboring edge on $\partial \delta_{m(j)}$.

For $E \in \mathcal{E}_{h}^{\delta_{m(j)}}\left(\partial \delta_{m(j)}\right)$, we specify appropriate weighting factors $\lambda_{E, E^{\prime}} \in \mathbb{R}$, $E^{\prime} \in \mathcal{E}_{h}^{\partial \delta_{m(j)}}(E)$ according to

$$
\lambda_{E, E^{\prime}} \geq 0 \quad, \quad \text { and } \sum_{E \in \mathcal{E}_{h}^{\delta_{m(j)}}\left(E^{\prime}\right)} \lambda_{E, E^{\prime}}=1, E^{\prime} \in \mathcal{E}_{h}\left(\partial \delta_{m(j)}\right)
$$

and define the basis field $\hat{\mathbf{q}}_{E}, E \in \mathcal{E}_{h}\left(\delta_{m(j)}\right)$ by means of

$$
\hat{\mathbf{q}}_{e}=\left\{\begin{array}{lrr}
\mathbf{q}_{E} & , & E \in \mathcal{E}_{h}\left(\delta_{m(j)}\right) \backslash \mathcal{E}_{h}^{\delta_{m(j)}}\left(\partial \delta_{m(j)}\right) \\
\mathbf{q}_{E}+\sum_{E^{\prime} \in \mathcal{E}_{h}^{\partial \delta_{m(j)}(E)}} \lambda_{E, E^{\prime}} \mathbf{q}_{e^{\prime}} & , & E \in \mathcal{E}_{h}^{\delta_{m(j)}}\left(\partial \delta_{m(j)}\right)
\end{array}\right.
$$

With regard to the thus specified basis fields we define

$$
\mathbf{M}_{\mathbf{h}}\left(\delta_{m(j)}\right):=\operatorname{span}\left\{\hat{\mathbf{q}}_{E} \mid E \in \mathcal{E}_{h}\left(\delta_{m(j)}\right)\right\} .
$$

The multiplier space $\mathbf{M}_{\mathbf{h}}(S)$ will be equipped with the mesh-dependent norm

$$
\left\|\boldsymbol{\mu}_{\mathbf{h}}\right\|_{\mathbf{M}_{\mathbf{h}}(S)}:=\left(\sum_{m=1}^{M}\left\|\left.\boldsymbol{\mu}_{b h}\right|_{\delta_{m(j)}}\right\|_{-1 / 2, h, \delta_{m(j)}}\right)^{1 / 2}
$$

where

$$
\left\|\left.\boldsymbol{\mu}_{\mathbf{h}}\right|_{\delta_{m(j)}}\right\|_{-1 / 2, h, \delta_{m(j)}}:=h^{1 / 2}\left\|\left.\boldsymbol{\mu}_{\mathbf{h}}\right|_{\delta_{m(j)}}\right\|_{0, \delta_{m(j)}} .
$$

The mortar edge element approximation of (3.1) then requires the solution of the saddle point problem:

Find $\left(\mathbf{j}_{\mathbf{h}}, \boldsymbol{\lambda}_{\mathbf{h}}\right) \in \mathbf{V}_{\mathbf{h}} \times \mathbf{M}_{\mathbf{h}}(S)$ such that

$$
\begin{aligned}
& a_{h}\left(\mathbf{j}_{\mathbf{h}}, \mathbf{q}_{\mathbf{h}}\right)+b_{h}\left(\mathbf{q}_{\mathbf{h}}, \boldsymbol{\lambda}_{\mathbf{h}}\right)=\ell\left(\mathbf{q}_{\mathbf{h}}\right), \quad \mathbf{q}_{\mathbf{h}} \in \mathbf{V}_{\mathbf{h}}, \\
& b_{h}\left(\mathbf{j}_{\mathbf{h}}, \boldsymbol{\mu}_{\mathbf{h}}\right) \quad=\quad 0 \quad, \quad \boldsymbol{\mu}_{\mathbf{h}} \in \mathbf{M}_{\mathbf{h}}(S),
\end{aligned}
$$

where the bilinear forms $a_{h}(\cdot, \cdot): \mathbf{V}_{\mathbf{h}} \times \mathbf{V}_{\mathbf{h}} \rightarrow \mathbb{R}$ and $b_{h}(\cdot, \cdot): \mathbf{V}_{\mathbf{h}} \times \mathbf{M}_{\mathbf{h}}(S) \rightarrow \mathbb{R}$ are given by the restriction of $a(\cdot, \cdot)$ and $b(\cdot, \cdot)$ to $\mathbf{V}_{\mathbf{h}} \times \mathbf{V}_{\mathbf{h}}$ and $\mathbf{V}_{\mathbf{h}} \times \mathbf{M}_{\mathbf{h}}(S)$, respectively.

We refer to $B_{h}: \mathbf{V}_{\mathbf{h}} \rightarrow \mathbf{M}_{\mathbf{h}}(S)^{*}$ as the operator associated with $b_{h}(\cdot, \cdot)$ according to

$$
b_{h}\left(\mathbf{q}_{\mathbf{h}}, \boldsymbol{\mu}_{\mathbf{h}}\right):=<\boldsymbol{\mu}_{\mathbf{h}},\left[\mathbf{q}_{\mathbf{h}} \wedge \mathbf{n}\right]>_{\frac{1}{2}, h, S}
$$

where

$$
<\cdot, \cdot>_{\frac{1}{2}, h, S}:=\sum_{m=1}^{M}<\cdot, \cdot>_{\frac{1}{2}, h, \delta_{m(j)}}
$$

and $\left\langle\cdot, \cdot>_{\frac{1}{2}, h, \delta_{m(j)}}\right.$ denotes the dual pairing between $\mathbf{M}_{\mathbf{h}}\left(\delta_{m(j)}\right)^{*}$ and $\mathbf{M}_{\mathbf{h}}\left(\delta_{m(j)}\right)$. 
In the sequel, we will derive the discrete counterparts of Lemma 3.2, Lemma 3.3, and Theorem 3.1. As a preparatory result we prove:

LEMMA 4.1. Let $E_{h}^{\delta_{m(j)}}: \mathbf{R T}_{0,0}\left(\delta_{m(j)} ; \mathcal{T}_{\delta_{m(j)}}\right) \rightarrow \mathbf{N d}_{1}\left(\Omega_{j} ; \mathcal{T}_{j}\right)$ be the extension operator given by

$$
\left(E_{h}^{\delta_{m(j)}} \boldsymbol{\lambda}_{\mathbf{h}}^{(j)}\right) \wedge \mathbf{n}:=\boldsymbol{\lambda}_{\mathbf{h}}^{(j)} \quad, \quad \boldsymbol{\lambda}_{\mathbf{h}}^{(j)} \in \mathbf{R} \mathbf{T}_{0,0}\left(\delta_{m(j)} ; \mathcal{T}_{\delta_{m(j)}}\right)
$$

where all degrees of freedom that are not located on $\delta_{m(j)}$ are set equal to zero. Then, for $\boldsymbol{\lambda}_{\mathbf{h}}^{(j)} \in \mathbf{R T}_{0,0}\left(\delta_{m(j)} ; \mathcal{T}_{\delta_{m(j)}}\right)$ there holds

$$
\left\|E_{h}^{\delta_{m(j)}} \boldsymbol{\lambda}_{\mathbf{h}}^{(j)}\right\|_{c u r l, \Omega_{j}} \leq C h_{\delta_{m(j)}}^{1 / 2}\left\|\boldsymbol{\lambda}_{\mathbf{h}}^{(j)}\right\|_{\operatorname{div}_{\tau}, \delta_{m(j)}}
$$

ProOF. Using affine equivalence and scaling arguments, it can be shown that for any $\mathbf{q}_{\mathbf{h}} \in \mathbf{N d}_{1}\left(\Omega_{i} ; \mathcal{T}_{i}\right)$, we have (cf. Lemma 4.2 in [69]):

$$
\begin{gathered}
\left.c h_{i}^{3} \sum_{T \in \mathcal{F}_{h}\left(\bar{\Omega}_{i}\right)}\left|\left(\mathbf{n}_{T} \cdot \operatorname{curl} \mathbf{q}_{\mathbf{h}}\right)\right|_{T}\right|^{2} \leq\left\|\mathbf{c u r l} \mathbf{q}_{\mathbf{h}}\right\|_{0, \Omega_{i}}^{2} \leq\left. C h_{i}^{3} \sum_{T \in \mathcal{F}_{h}\left(\bar{\Omega}_{i}\right)}\left|\left(\mathbf{n}_{T} \cdot \mathbf{c u r l} \mathbf{q}_{\mathbf{h}}\right)\right|_{T}\right|^{2}, \\
\quad h_{i}^{3} \sum_{E \in \mathcal{E}_{h}\left(\bar{\Omega}_{i}\right)}\left|\left(\mathbf{t}_{E} \cdot \mathbf{q}_{\mathbf{h}}\right)\left(x_{E}^{M}\right)\right|^{2} \leq\left\|\mathbf{q}_{\mathbf{h}}\right\|_{0, \Omega_{i}}^{2} \leq C h_{i}^{3} \sum_{E \in \mathcal{E}_{h}\left(\bar{\Omega}_{i}\right)}\left|\left(\mathbf{t}_{E} \cdot \mathbf{q}_{\mathbf{h}}\right)\left(x_{E}^{M}\right)\right|^{2} .
\end{gathered}
$$

Similarly, for $\mathbf{q}_{\mathbf{h}} \in \mathbf{R} \mathbf{T}_{0}\left(\delta_{m(j)} ; \mathcal{T}_{\delta_{m(j)}}\right)$, we have

$$
\begin{aligned}
\left.\operatorname{ch}_{\delta_{m(j)}}^{2} \sum_{T \in \mathcal{T}_{\delta_{m(j)}}}\left|\left(\operatorname{div}_{\tau} \mathbf{q}_{\mathbf{h}}\right)\right|_{T}\right|^{2} & \leq\left\|\operatorname{div}_{\tau} \mathbf{q}_{\mathbf{h}}\right\|_{0, \delta_{m(j)}}^{2} \\
& \leq\left. C h_{\delta_{m(j)}}^{2} \sum_{T \in \mathcal{T}_{\delta_{m}(j)}}\left|\left(\operatorname{div}_{\tau} \mathbf{q}_{\mathbf{h}}\right)\right|_{T}\right|^{2}, \\
c h_{\delta_{m(j)}}^{2} \sum_{E \in \mathcal{E}_{h}\left(\bar{\delta}_{m(j)}\right)}\left|\left(\mathbf{n}_{E} \cdot \mathbf{q}_{\mathbf{h}}\right)\left(x_{E}^{M}\right)\right|^{2} & \leq\left\|\mathbf{q}_{\mathbf{h}}\right\|_{0, \delta_{m(j)}}^{2} \\
& \leq C h_{\delta_{m(j)}}^{2} \sum_{E \in \mathcal{E}_{h}\left(\bar{\delta}_{m(j)}\right)}\left|\left(\mathbf{n}_{E} \cdot \mathbf{q}_{\mathbf{h}}\right)\left(x_{E}^{M}\right)\right|^{2} .
\end{aligned}
$$

In view of (4.25) and the previous inequalities, we get

$$
\begin{aligned}
\left\|\operatorname{curl}\left(E_{h}^{\delta_{m(j)}} \boldsymbol{\lambda}_{\mathbf{h}}^{(j)}\right)\right\|_{0, \Omega_{j}}^{2} & \left.\leq C h_{j}^{3} \sum_{T \in \mathcal{T}_{\delta_{m(j)}}} \mid \mathbf{n}_{T} \cdot \operatorname{curl}\left(E_{h}^{\delta_{m(j)}} \boldsymbol{\lambda}_{\mathbf{h}}^{(j)}\right)\right)\left.\left.\right|_{T}\right|^{2} \\
& =\left.C h_{j}^{3} \sum_{T \in \mathcal{T}_{\delta_{m(j)}}}\left|\operatorname{div}_{\tau}\left(E_{h}^{\delta_{m(j)}} \boldsymbol{\lambda}_{\mathbf{h}}^{(j)} \wedge \mathbf{n}\right)\right|_{T}\right|^{2} \\
& =\left.C h_{j}^{3} \sum_{T \in \mathcal{T}_{\delta_{m(j)}}}\left|\operatorname{div}_{\tau}\left(\boldsymbol{\lambda}_{\mathbf{h}}^{(j)}\right)\right|_{T}\right|^{2} \\
& \leq C h_{j}\left\|\operatorname{div}_{\tau}\left(\boldsymbol{\lambda}_{\mathbf{h}}^{(j)}\right)\right\|_{0, \delta_{m(j)}}^{2}
\end{aligned}
$$


and

$$
\begin{aligned}
\left\|E_{h}^{\delta_{m(j)}} \boldsymbol{\lambda}_{\mathbf{h}}^{(j)}\right\|_{0, \Omega_{j}}^{2} & \leq C h_{j}^{3} \sum_{E \in \mathcal{E}_{h}\left(\bar{\Omega}_{j}\right)}\left|\left(\mathbf{t}_{E} \cdot E_{h}^{\delta_{m(j)}} \boldsymbol{\lambda}_{\mathbf{h}}^{(j)}\right)\left(x_{E}^{M}\right)\right|^{2} \\
& =C h_{j}^{3} \sum_{E \in \mathcal{E}_{h}\left(\bar{\Omega}_{j}\right)}\left|\mathbf{n}_{E} \cdot\left(E_{h}^{\delta_{m(j)}} \boldsymbol{\lambda}_{\mathbf{h}}^{(j)} \wedge \mathbf{n}\right)\left(x_{E}^{M}\right)\right|^{2} \\
& =C h_{j}^{3} \sum_{E \in \mathcal{E}_{h}\left(\delta_{m(j)}\right)}\left|\left(\mathbf{n}_{E} \cdot \boldsymbol{\lambda}_{\mathbf{h}}^{(j)}\right)\left(x_{E}^{M}\right)\right|^{2} \\
& \leq h_{j}\left\|\boldsymbol{\lambda}_{\mathbf{h}}^{(j)}\right\|_{0, \delta_{m(j)}}^{2},
\end{aligned}
$$

which proves (4.26).

Lemma 4.2. The bilinear form $a_{h}(\cdot, \cdot)$ is elliptic on the kernel of $B_{h}$ uniformly in $h$, i.e., there exists a positive constant $\alpha \in \mathbb{R}$ such that

$$
a_{h}\left(\mathbf{q}_{\mathbf{h}}, \mathbf{q}_{\mathbf{h}}\right) \geq \alpha\left\|\mathbf{q}_{\mathbf{h}}\right\|_{\mathbf{V}_{\mathbf{h}}}^{2} \quad, \quad \mathbf{q}_{h} \in \operatorname{Ker} B_{h} .
$$

Proof. Since $\left[\mathbf{q}_{\mathbf{h}} \wedge \mathbf{n}\right]=0$ on $\gamma_{m}, 1 \leq m \leq M$, we have Ker $B_{h}=\mathbf{H}_{\mathbf{0}}(\mathbf{c u r l} ; \Omega)$.

LEmma 4.3. The bilinear form $b_{h}(\cdot, \cdot)$ satisfies the inf-sup condition

$$
\inf _{\boldsymbol{\mu} \in \mathbf{M}_{\mathbf{h}}(S)} \sup _{\mathbf{q u}_{\mathbf{h}} \in \mathbf{V}_{\mathbf{h}}} \frac{b_{h}\left(\mathbf{q}_{\mathbf{h}}, \boldsymbol{\mu}\right)}{\left\|\mathbf{q}_{\mathbf{h}}\right\|_{\mathbf{V}_{\mathbf{h}}}\|\boldsymbol{\mu}\|_{\mathbf{M}_{\mathbf{h}}(S)}} \geq \beta>0 .
$$

Proof. For any $\boldsymbol{\mu}_{\mathbf{h}} \in \mathbf{M}_{\mathbf{h}}(S)$ we define $\mathbf{p}_{\mathbf{h}}^{(j)} \in \mathbf{R T}_{0,0}\left(\delta_{m(j)} ; \mathcal{T}_{\delta_{m(j)}}\right)$ according to

$$
\int_{E} \mathbf{t}_{E} \cdot \mathbf{p}_{\mathbf{h}}^{(j)} d \sigma=\int_{E} \mathbf{t}_{E} \cdot \boldsymbol{\mu}_{\mathbf{h}} d \sigma \quad, \quad E \in \mathcal{E}_{h}\left(\delta_{m(j)}\right)
$$

and refer to $\mathbf{q}_{\mathbf{h}}^{(j)} \in \mathbf{N d}_{1}\left(\Omega_{j} ; \mathcal{T}_{j}\right)$ as the trivial extension, i.e.,

$$
\mathbf{q}_{\mathbf{h}}^{(j)} \wedge \mathbf{n}=\mathbf{p}_{\mathbf{h}}^{(j)} \text { on } \delta_{m(j)},
$$

where all degrees of freedom that are not located on $\delta_{m(j)}$ are set equal to zero.

In particular, we have

$$
\left[\mathbf{q}_{\mathbf{h}}^{(j)} \wedge \mathbf{n}\right]=\mathbf{p}_{\mathbf{h}}^{(j)} .
$$

In view of Lemma 4.1 it follows that

$$
\begin{aligned}
\left\|\mathbf{q}_{\mathbf{h}}^{(j)}\right\|_{c u r l, \Omega_{j}} & \leq C h_{j}^{\frac{1}{2}}\left\|\mathbf{p}_{\mathbf{h}}^{(j)}\right\|_{d i v_{\tau}, \delta_{m(j)}} \\
& \leq C h_{j}^{-\frac{1}{2}}\left\|\mathbf{p}_{\mathbf{h}}^{(j)}\right\|_{0, \delta_{m(j)}} \\
& =C h_{j}^{-\frac{1}{2}}\left\|\left[\mathbf{q}_{h}^{(j)} \wedge \mathbf{n}\right]\right\|_{0, \delta_{m(j)}} .
\end{aligned}
$$

Moreover, taking advantage of the inf-sup condition

$$
\inf _{\boldsymbol{\mu}_{\mathbf{h}} \in \mathbf{M}_{\mathbf{h}}\left(\delta_{m(j)}\right)} \sup _{\mathbf{q}_{\mathbf{h}} \in \mathbf{R} \mathbf{T}_{0,0}\left(\delta_{m(j)} ; \mathcal{T}_{\delta_{m(j)}}\right)} \frac{\left(\mathbf{q}_{\mathbf{h}}, \boldsymbol{\mu}_{\mathbf{h}}\right)_{0, \delta_{m(j)}}}{\left\|\mathbf{q}_{\mathbf{h}}\right\|_{0, \delta_{m(j)}}\left\|\boldsymbol{\mu}_{\mathbf{h}}\right\|_{0, \delta_{m(j)}}} \geq \beta>0
$$


from Lemma 3.20 in [38] and (4.30), we obtain

$$
\begin{aligned}
\left(\boldsymbol{\mu}_{\mathbf{h}},\left.\left[\mathbf{q}_{\mathbf{h}}^{(j)} \wedge \mathbf{n}\right]\right|_{\delta_{m(j)}}\right)_{0, \delta_{m(j)}} & \geq C\left\|\boldsymbol{\mu}_{\mathbf{h}}\right\|_{0, \delta_{m(j)}}\left\|\left[\mathbf{q}_{\mathbf{h}}^{(j)} \wedge \mathbf{n}\right]\right\|_{0, \delta_{m(j)}} \\
& \geq C h_{j}^{\frac{1}{2}}\left\|\boldsymbol{\mu}_{\mathbf{h}}\right\|_{0, \delta_{m(j)}}\left\|\mathbf{q}_{\mathbf{h}}^{(j)}\right\|_{c u r l, \Omega_{j}} \\
& \geq C\left\|\boldsymbol{\mu}_{\mathbf{h}}\right\|_{-\frac{1}{2}, h, \delta_{m(j)}}\left\|\mathbf{q}_{\mathbf{h}}^{(j)}\right\|_{c u r l, \Omega_{j}} .
\end{aligned}
$$

On the other hand,

$$
\begin{aligned}
(4.32)\left(\boldsymbol{\mu}_{\mathbf{h}},\left.\left[\mathbf{q}_{\mathbf{h}}^{(j)} \wedge \mathbf{n}\right]\right|_{\delta_{m(j)}}\right)_{0, \delta_{m(j)}} & \geq C\left\|\mu_{h}\right\|_{0, \delta_{m(j)}}\left\|\left[\mathbf{q}_{h}^{(j)} \wedge \mathbf{n}\right]\right\|_{0, \delta_{m(j)}} \\
& =C h_{j}^{\frac{1}{2}}\left\|\boldsymbol{\mu}_{\mathbf{h}}\right\|_{0, \delta_{m(j)}} h_{j}^{-\frac{1}{2}}\left\|\left[\mathbf{q}_{\mathbf{h}}^{(j)} \wedge \mathbf{n}\right]\right\|_{0, \delta_{m(j)}} \\
& =C\left\|\boldsymbol{\mu}_{\mathbf{h}}\right\|_{-\frac{1}{2}, h, \delta_{m(j)}}\left\|\left[\mathbf{q}_{\mathbf{h}}^{(j)} \wedge \mathbf{n}\right]\right\|_{\frac{1}{2}, h, \delta_{m(j)}} .
\end{aligned}
$$

The assertion follows by adding (4.31), (4.32) and summing over all $1 \leq m \leq M$.

As an immediate consequence of Lemma 4.2 and Lemma 4.3 we obtain:

THEOREM 4.1. The mortar edge element approximation (4.22) admits a unique $\operatorname{solution}\left(\mathbf{j}_{\mathbf{h}}, \boldsymbol{\lambda}_{\mathbf{h}}\right) \in \mathbf{V}_{\mathbf{h}} \times \mathbf{M}_{\mathbf{h}}(S)$.

The mortar finite element approximation of the equation (3.32), satisfied by the irrotational part of the solution, is fairly standard (cf., e.g., [68] and the references therein). In particular, following [21], we introduce the product space

$$
\begin{aligned}
V_{h} & :=\left\{v_{h} \in L^{2}(\Omega)\left|v_{h}\right|_{\Omega_{i}} \in S_{1, \Gamma}\left(\Omega_{i} ; \mathcal{T}_{i}\right), 1 \leq i \leq N\right\}, \\
\left\|v_{h}\right\|_{V_{h}} & :=\left(\left\|v_{h}\right\|_{X}^{2}+\left\|\left.\left[v_{h}\right]\right|_{S}\right\|_{\frac{1}{2}, h, S}^{2}\right)^{1 / 2}
\end{aligned}
$$

and the multiplier space

$$
\begin{aligned}
& M_{h}(S):=\prod_{m=1}^{M} M_{h}\left(\delta_{m(j)}\right) \\
& S_{1,0}\left(\delta_{m(j)} ; \mathcal{T}_{\delta_{m(j)}}\right) \subset M_{h}\left(\delta_{m(j)}\right), \\
& \operatorname{dim} M_{h}\left(\delta_{m(j)}\right)=\operatorname{dim} S_{1,0}\left(\delta_{m(j)} ; \delta_{m(j)}\right) .
\end{aligned}
$$

For the precise definition of $M_{h}\left(\delta_{m(j)}\right)$ we refer to [20].

The multiplier space $M_{h}(S)$ will be equipped with the mesh-dependent norm

$$
\left\|\mu_{h}\right\|_{M_{h}(S)}:=\left(\sum_{m=1}^{M}\left\|\left.\mu_{h}\right|_{\delta_{m(j)}}\right\|_{-1 / 2, h, \delta_{m(j)}}\right)^{1 / 2} .
$$

Then, the mortar edge element approximation of (3.32) can be stated as follows:

Find $\left(u_{h}, \lambda_{h}\right) \in V_{h} \times M_{h}(S)$ such that

$$
\begin{aligned}
& c_{h}\left(u_{h}, v_{h}\right)+d_{h}\left(v_{h}, \lambda_{h}\right)=r\left(v_{h}\right), \quad v_{h} \in V_{h}, \\
& d_{h}\left(u_{h}, \mu_{h}\right) \quad=\quad 0 \quad, \quad \mu_{h} \in M_{h}(S) \text {, }
\end{aligned}
$$

where the bilinear forms $c_{h}(\cdot, \cdot): V_{h} \times V_{h} \rightarrow \mathbb{R}$ and $d_{h}(\cdot, \cdot): V_{h} \times M_{h}(S) \rightarrow \mathbb{R}$ are given by the restriction of $c(\cdot, \cdot)$ and $d(\cdot, \cdot)$ to $V_{h} \times V_{h}$ and $V_{h} \times M_{h}(S)$, respectively, and the functional $r_{h}: V_{h} \rightarrow \mathbb{R}$ is given by

(4.40) $r_{h}\left(v_{h}\right):=\sum_{i=1}^{N} \int_{\Omega_{i}} \mathbf{f} \cdot \operatorname{grad} v_{h} d \mathbf{x}-a_{h}\left(\mathbf{j}_{\mathbf{h}}, \operatorname{grad} v_{h}\right) \quad, \quad v_{h} \in V_{h}$. 
We refer to $D_{h}: V_{h} \rightarrow M_{h}(S)^{*}$ as the operator associated with $d_{h}(\cdot, \cdot)$. As the discrete counterparts of Lemma 3.4 and Theorem 3.2 it can be shown that the following results hold true.

Lemma 4.4. The bilinear form $d_{h}(\cdot, \cdot)$ is elliptic on the kernel of $D_{h}$ uniformly in $h$, i.e., there exists a positive constant $\alpha \in \mathbb{R}$ such that

$$
d_{h}\left(v_{h}, v_{h}\right) \geq \alpha\left\|v_{h}\right\|_{V_{h}}^{2}, \quad v_{h} \in \operatorname{Ker} D_{h},
$$

and the bilinear form $d_{h}(\cdot, \cdot)$ satisfies the inf-sup condition

$$
\inf _{\mu \in M_{h}(S)} \sup _{v_{h} \in V_{h}} \frac{d_{h}\left(v_{h}, \mu\right)}{\left\|v_{h}\right\|_{V_{h}}\|\mu\|_{M_{h}(S)}} \geq \beta>0 .
$$

Proof. We refer to [21].

A direct consequence of Lemma 4.4 is:

THEOREM 4.2. The mortar finite element approximation (4.37) admits a unique solution $\left(u_{h}, \lambda_{h}\right) \in V_{h} \times M_{h}(S)$.

A priori error estimates for the discretization errors

$$
\mathbf{e}_{\mathbf{j}}:=\mathbf{j}-\mathbf{j}_{\mathbf{h}} \quad, \quad \mathbf{e}_{\boldsymbol{\lambda}}:=\boldsymbol{\lambda}-\boldsymbol{\lambda}_{\mathbf{h}}
$$

in the mortar edge element approximation of the eddy currents equations have been derived in $[12,69]$. Here, we report on the optimal estimates that have been established in [69].

Theorem 4.3. Let $(\mathbf{j}, \boldsymbol{\lambda}) \in \mathbf{V} \times \mathbf{M}(S)$ be the solution of the macro-hybrid formulation (3.16) of the eddy currents equations and assume that $\mathbf{j} \in \mathbf{H}^{\mathbf{1}}(\mathbf{c u r l} ; \Omega)$, where

$$
\mathbf{H}^{\mathbf{1}}(\operatorname{curl} ; \Omega):=\left\{\mathbf{q} \in \mathbf{H}^{\mathbf{1}}(\Omega) \mid \operatorname{curl} \mathbf{q} \in \mathbf{H}^{\mathbf{1}}(\Omega)\right\} .
$$

Let further $\left(\mathbf{j}_{\mathbf{h}}, \boldsymbol{\lambda}_{\mathbf{h}}\right) \in \mathbf{V}_{\mathbf{h}} \times \mathbf{M}_{\mathbf{h}}(S)$ be the solution of the mortar edge element approximation (4.22). Then, there exist positive constants $C_{\nu}, 1 \leq \nu \leq 2$, depending on the shape regularity of the triangulations $\mathcal{T}_{i}, 1 \leq i \leq N$, such that for the discretization errors $\mathbf{e}_{\mathbf{j}}$ and $\mathbf{e}_{\boldsymbol{\lambda}}$ there holds

$$
\begin{aligned}
&\left\|\mathbf{e}_{\mathbf{j}}\right\|_{\mathbf{V}} \leq C_{1}\left(\sum_{i=1}^{N} h_{i}^{2}\|\mathbf{j}\|_{1, \text { curl }, \Omega_{i}}^{2}\right)^{1 / 2}, \\
&\left\|\mathbf{e}_{\boldsymbol{\lambda}}\right\|_{\mathbf{M}(S)} \leq C_{2}\left(\sum_{i=1}^{N} h_{i}^{2}\|\mathbf{j}\|_{1, \text { curl }, \Omega_{i}}^{2}\right)^{1 / 2} .
\end{aligned}
$$

Proof. We refer to Theorem 4.3 and Theorem 5.2 in [69].

The mortar edge element approximation is a nonconforming approach. Using duality techniques known from nonconforming finite element approximations, the error estimate (4.45) implies:

Corollary 4.1. Under the same assumptions as in Theorem 4.3 there holds

$$
\left\|\mathbf{e}_{\mathbf{j}}\right\|_{0, \Omega} \leq C\left(\sum_{i=1}^{N} h_{i}^{2}\|\mathbf{j}\|_{1, \text { curl }, \Omega_{i}}^{2}\right)^{1 / 2} .
$$




\section{Multilevel iterative solution methods}

An essential feature of the efficient iterative solution of the edge element discretized eddy currents equations is the appropriate treatment of the nontrivial kernel of the discrete curl operator. We will first discuss this issue for an edge element discretization with respect to a shape regular simplicial triangulation $\mathcal{T}_{h}$ of the computational domain $\Omega$, then consider a multilevel approach and finally address mortar edge element approximations.

In case of a single grid approximation, the problem is to compute $\mathbf{j}_{\mathbf{h}} \in$ $\mathbf{N d}_{1, \Gamma}\left(\Omega ; \mathcal{T}_{h}\right)$ such that

$$
\int_{\Omega}\left(\chi \operatorname{curl} \mathbf{j}_{\mathbf{h}} \cdot \operatorname{curl} \mathbf{q}_{\mathbf{h}}+\boldsymbol{\sigma} \mathbf{j}_{\mathbf{h}} \cdot \mathbf{q}_{\mathbf{h}}\right) d \mathbf{x}=\int_{\Omega} \mathbf{f} \cdot \mathbf{q}_{\mathbf{h}} d \mathbf{x}, \mathbf{q}_{\mathbf{h}} \in \mathbf{N d}_{1,0}\left(\Omega ; \mathcal{T}_{h}\right) .
$$

Assuming $\mathbf{N d}_{1,0}\left(\Omega ; \mathcal{T}_{h}\right)=\operatorname{span}\left\{\mathbf{q}_{E}^{(\nu)} \mid 1 \leq \nu \leq n_{h}\right\}$, we define $\mathbf{A}_{\mathbf{h}}=\left(a_{\nu \mu}\right)_{\nu, \mu=1}^{n_{h}}$ and $\mathbf{b}_{\mathbf{h}}=\left(b_{1}, \ldots, b_{n_{h}}\right)^{T}$ by means of

$$
\begin{aligned}
a_{\nu \mu} & :=\int_{\Omega}\left(\chi \operatorname{curl} \mathbf{q}_{E}^{(\nu)} \cdot \operatorname{curl} \mathbf{q}_{E}^{(\mu)}+\boldsymbol{\sigma} \mathbf{q}_{E}^{(\nu)} \cdot \mathbf{q}_{E}^{(\mu)}\right) d \mathbf{x}, 1 \leq \nu, \mu \leq n_{h}, \\
b_{\nu} & :=\int_{\Omega} \mathbf{f} \cdot \mathbf{q}_{E}^{(\nu)} d \mathbf{x}, 1 \leq \nu \leq n_{h} .
\end{aligned}
$$

Consequently, identifying the edge element function $\mathbf{j}_{\mathbf{h}} \in \mathbf{N d}_{1, \Gamma}\left(\Omega ; \mathcal{T}_{h}\right)$ with a vector $\mathbf{j}_{\mathbf{h}} \in \mathbb{R}^{n_{h}}$, the algebraic form of (5.1) can be written as

$$
\mathbf{A}_{\mathbf{h}} \mathbf{j}_{\mathbf{h}}=\mathbf{b}_{\mathbf{h}} \text {. }
$$

In particular, the matrix $\mathbf{A}_{\mathbf{h}}$ can be split according to

$$
\mathbf{A}_{\mathbf{h}}=\mathbf{S}_{\mathbf{h}}+\mathbf{T}_{\mathbf{h}}
$$

where the stiffness matrix $\mathbf{S}_{\mathbf{h}}=\left(s_{\nu \mu}\right)_{\nu, \mu=1}^{n_{h}}$ and the mass matrix $\mathbf{T}_{\mathbf{h}}=\left(t_{\nu \mu}\right)_{\nu, \mu=1}^{n_{h}}$ are given by

$$
\begin{aligned}
s_{\nu \mu} & :=\int_{\Omega} \chi \operatorname{curl} \mathbf{q}_{E}^{(\nu)} \cdot \operatorname{curl} \mathbf{q}_{E}^{(\mu)} d \mathbf{x}, 1 \leq \nu, \mu \leq n_{h}, \\
t_{\nu \mu} & \left.:=\int_{\Omega} \sigma \mathbf{q}_{E}^{(\nu)} \cdot \mathbf{q}_{E}^{(\mu)}\right) d \mathbf{x}, 1 \leq \nu, \mu \leq n_{h} .
\end{aligned}
$$

Hence, (5.2) takes the form

$$
\left(\mathbf{S}_{\mathbf{h}}+\mathbf{T}_{\mathbf{h}}\right) \mathbf{j}_{\mathbf{h}}=\mathbf{b}_{\mathbf{h}}
$$

We note that the stiffness matrix $\mathbf{S}_{\mathbf{h}}$ has a nontrivial kernel due the kernel of the discrete curl operator.

The following simple example illustrates the possible bad convergence behavior of classical iterative schemes in case of the occurrence of a nontrivial kernel: We consider the linear algebraic system

$$
\left(\mathbf{S}_{\mathbf{h}}+\varepsilon \mathbf{T}_{\mathbf{h}}\right) \mathbf{x}_{\mathbf{h}}=\mathbf{b}_{\mathbf{h}},
$$

where

$$
\mathbf{S}_{\mathbf{h}}:=\left(\begin{array}{rr}
1 & -1 \\
-1 & 1
\end{array}\right) \quad, \quad \mathbf{T}_{\mathbf{h}}:=\left(\begin{array}{ll}
1 & 0 \\
0 & 1
\end{array}\right)
$$


and $\varepsilon>0$. The matrix $\mathbf{S}_{\mathbf{h}}$ has the eigenvalues $\lambda_{h}^{(1)}=0$ and $\lambda_{h}^{(2)}=2$ with the associated eigenvectors $\mathbf{v}_{\mathbf{h}}^{(1)}=(1,1)^{T}$ and $\mathbf{v}_{\mathbf{h}}^{(2)}=(1,-1)^{T}$.

In case of the solution of (5.4) by the damped Jacobi iteration with damping parameter $\omega_{1}>0$, the error $\mathbf{e}_{\mathbf{h}}^{(\nu)}:=\mathbf{x}_{\mathbf{h}}-\mathbf{x}_{\mathbf{h}}^{(k)}$ with respect to the $k$-th iterate $\mathbf{x}_{\mathbf{h}}^{(k)} \in \mathbb{R}^{2}$ satisfies

$$
\mathbf{e}_{\mathbf{h}}^{(k+1)}=\mathbf{M}_{\mathbf{h}} \mathbf{e}_{\mathbf{h}}^{(k)}
$$

where

$$
\mathbf{M}_{\mathbf{h}}:=\mathbf{I}_{\mathbf{h}}-\frac{\omega_{1}}{1+\varepsilon}\left(\mathbf{S}_{\mathbf{h}}+\varepsilon \mathbf{T}_{\mathbf{h}}\right) .
$$

Splitting the error according to

$$
\mathbf{e}_{\mathbf{h}}^{(k)}=\alpha_{k}^{(1)} \mathbf{v}_{\mathbf{h}}^{(1)}+\alpha_{k}^{(2)} \mathbf{v}_{\mathbf{h}}^{(2)},
$$

and observing

$$
\begin{aligned}
& \mathbf{M}_{\mathbf{h}} \mathbf{v}_{\mathbf{h}}^{(1)}=\left(1-\omega_{1} \frac{\varepsilon}{1+\varepsilon}\right) \mathbf{v}_{\mathbf{h}}^{(1)} \approx\left(1-\omega_{1} \varepsilon\right) \mathbf{v}_{\mathbf{h}}^{(1)} \\
& \mathbf{M}_{\mathbf{h}} \mathbf{v}_{\mathbf{h}}^{(2)}=\left(1-\omega_{1} \frac{2 \varepsilon+1}{1+\varepsilon}\right) \mathbf{v}_{\mathbf{h}}^{(1)} \approx\left(1-2 \omega_{1} \varepsilon\right) \mathbf{v}_{\mathbf{h}}^{(2)}
\end{aligned}
$$

we see that the component $\alpha_{k}^{(1)} \mathbf{v}_{\mathbf{h}}^{(1)}$, living in the kernel of $\mathbf{S}_{\mathbf{h}}$, experiences a very bad damping in case $\varepsilon \ll 1$, whereas the other component $\alpha_{k}^{(2)} \mathbf{v}_{\mathbf{h}}^{(2)}$, living in the orthogonal subspace, is very well damped, if the damping parameter is chosen according to $\omega_{1} \approx 0.5$.

A convenient remedy to improve the convergence of the iterative process is to perform a defect correction on the kernel of $\mathbf{S}_{\mathbf{h}}$. We denote by

$$
\begin{aligned}
\mathbf{d}_{\mathbf{h}}^{(\nu)} & =\mathbf{b}_{\mathbf{h}}-\left(\mathbf{S}_{\mathbf{h}}+\varepsilon \mathbf{T}_{\mathbf{h}}\right) \mathbf{x}_{\mathbf{h}}^{(k+1 / 2)} \\
\mathbf{x}_{\mathbf{h}}^{(k+1 / 2)} & =\left(\mathbf{I}_{\mathbf{h}}-\frac{\omega_{1}}{1+\varepsilon}\left(\mathbf{S}_{\mathbf{h}}+\varepsilon \mathbf{T}_{\mathbf{h}}\right)\right) \mathbf{x}_{\mathbf{h}}^{(k)}
\end{aligned}
$$

the defect with respect to the iterate $\mathbf{x}_{\mathbf{h}}^{(k+1 / 2)}$ obtained by applying one step of the damped Jacobi iteration to (5.4) with $\mathbf{x}_{\mathbf{h}}^{(k)}$ as startiterate.

Further, we set

$$
\begin{aligned}
\mathbf{Z}_{\mathbf{h}} & :=\left(\mathbf{v}_{\mathbf{h}}^{(1)}\right)^{T}\left(\mathbf{S}_{\mathbf{h}}+\varepsilon \mathbf{T}_{\mathbf{h}}\right) \mathbf{v}_{\mathbf{h}}^{(1)}, \\
r_{h}^{(k)} & :=\left(\mathbf{v}_{\mathbf{h}}^{(1)}\right)^{T} \mathbf{d}_{\mathbf{h}}^{(k)} .
\end{aligned}
$$

The scalar defect correction takes the form

$$
\mathbf{Z}_{\mathbf{h}} \mathbf{Y}_{\mathbf{h}}=r_{h}^{(k)}
$$

and results in the new iterate

$$
\mathbf{x}_{\mathbf{h}}^{(\nu+1)}=\mathbf{x}_{\mathbf{h}}^{(k+1 / 2)}+\omega_{2} \mathbf{y}_{\mathbf{h}}=\mathbf{x}_{\mathbf{h}}^{(k+1 / 2)}+\omega_{2} \mathbf{Z}_{\mathbf{h}}^{-1} r_{h}^{(k)} \mathbf{v}_{\mathbf{h}}^{(1)},
$$

where the correction has been damped by the factor $\omega_{2}>0$.

It follows from (5.7) that the associated iteration operator $\hat{\mathbf{M}}_{\mathbf{h}}$ is given by

$$
\hat{\mathbf{M}}_{\mathbf{h}}=\left(\mathbf{I}_{\mathbf{h}}-\omega_{2} \mathbf{v}_{\mathbf{h}}^{(1)} \mathbf{Z}_{\mathbf{h}}^{-1}\left(\mathbf{v}_{\mathbf{h}}^{(1)}\right)^{T}\left(\mathbf{S}_{\mathbf{h}}+\varepsilon \mathbf{T}_{\mathbf{h}}\right)\right)\left(\mathbf{I}_{\mathbf{h}}-\frac{\omega_{1}}{1+\varepsilon}\left(\mathbf{S}_{\mathbf{h}}+\varepsilon \mathbf{T}_{\mathbf{h}}\right)\right) .
$$


In particular, we obtain

$$
\begin{aligned}
& \hat{\mathbf{M}}_{\mathbf{h}} \mathbf{v}_{\mathbf{h}}^{(1)}=\left(1-\omega_{2}\right)\left(1-\omega_{1} \frac{\varepsilon}{1+\varepsilon}\right) \mathbf{v}_{\mathbf{h}}^{(1)}, \\
& \hat{\mathbf{M}}_{\mathbf{h}} \mathbf{v}_{\mathbf{h}}^{(2)}=\left(1-\omega_{1} \frac{2+\varepsilon}{1+\varepsilon}\right) \mathbf{v}_{\mathbf{h}}^{(2)},
\end{aligned}
$$

which shows that excellent damping properties for both components of the error can be achieved.

We note that for edge element discretizations (5.1) of the eddy currents equations the nontrivial kernel of the discrete curl operator is given by the subspace of irrotational vector fields spanned by the gradients of the conforming P1 finite elements. This means that the defect correction has to be performed in that subspace. In particular, the defect correction problem takes the form

$$
\int_{\Omega} \boldsymbol{\sigma g r a d} \varphi_{h} \cdot \operatorname{grad} v_{h} d \mathbf{x}=r\left(v_{h}\right) \quad, \quad v_{h} \in S_{1,0}\left(\Omega ; \mathcal{T}_{h}\right)
$$

where $r(\cdot)$ is the residual

$$
r\left(v_{h}\right):=\ell\left(\operatorname{grad} v_{h}\right)-a_{h}\left(\mathbf{j}_{\mathbf{h}}^{(k+1 / 2)}, \operatorname{grad} v_{h}\right) \quad, \quad v_{h} \in S_{1,0}\left(\Omega ; \mathcal{T}_{h}\right)
$$

Assuming $S_{1, \Gamma}\left(\Omega ; \mathcal{T}_{h}\right)=\operatorname{span}\left\{\varphi_{h}^{(\nu)} \mid 1 \leq \nu \leq m_{h}\right\}$, we define the stiffness matrix $\mathbf{C}_{\mathbf{h}}=\left(c_{\nu \mu}\right)_{\nu, \mu=1}^{m_{h}}$ and the vector $\mathbf{r}_{\mathbf{h}}=\left(r_{1}, \ldots r_{m_{h}}\right)^{T}$ according to

$$
\begin{aligned}
c_{\nu \mu} & :=\int_{\Omega} \boldsymbol{\sigma g r a d} \varphi_{h}^{(\nu)} \cdot \operatorname{grad} \varphi_{h}^{(\mu)} d \mathbf{x}, 1 \leq \nu, \mu \leq m_{h}, \\
r_{\nu} & :=r\left(\varphi_{h}^{(\nu)}\right), 1 \leq \nu \leq m_{h} .
\end{aligned}
$$

Identifying $\varphi_{h} \in S_{1,0}\left(\Omega ; \mathcal{T}_{h}\right)$ with a vector $\varphi_{\mathbf{h}} \in \mathbb{R}^{m_{h}}$, the algebraic form of the defect equation (5.8) is given by

$$
\mathrm{C}_{\mathbf{h}} \varphi_{\mathbf{h}}=\mathbf{r}_{\mathbf{h}}
$$

Altogether, this gives rise to a hybrid iterative process, consisting of the application of a classical iterative method to the fully edge element discretized problem and the defect correction problem. In particular, given an iterate $\mathbf{j}_{\mathbf{h}}^{(k)}, k \geq 0$, a cycle of the hybrid iterative solver is as follows:

\section{Step 1: SSOR sweep on the edge element discretized problem}

Compute $\mathbf{j}_{\mathbf{h}}^{(k+1 / 2)} \in \mathbb{R}^{n_{h}}$ by the application of the symmetric SOR method to

$$
\mathbf{A}_{\mathbf{h}} \mathbf{j}_{\mathbf{h}}=\mathbf{b}_{\mathbf{h}}
$$

using $\mathbf{j}_{\mathbf{h}}^{(0)}=\mathbf{j}_{\mathbf{h}}^{(k)}$ as a startiterate.

Step 2: Correctional SSOR sweep on the defect correction problem

Compute $\varphi_{\mathbf{h}}^{(k+1 / 2)} \in \mathbb{R}^{m_{h}}$ by the application of the symmetric SOR scheme to

$$
\mathrm{C}_{\mathbf{h}} \varphi_{\mathbf{h}}=\mathbf{r}_{\mathbf{h}}
$$

using $\varphi_{\mathbf{h}}^{(0)}=\mathbf{0}$ as a startiterate.

Step 3: Additive correction 
Denoting by $\boldsymbol{\psi}_{\mathbf{h}}^{(k+1 / 2)} \in \mathbb{R}^{n_{h}}$ the vector representing the projection of $\operatorname{grad} \varphi_{h}^{(k+1 / 2)}$, $\varphi_{h}^{(k+1 / 2)} \in S_{1, \Gamma}\left(\Omega ; \mathcal{T}_{h}\right)$ onto $\mathbf{N d}_{1, \Gamma}\left(\Omega ; \mathcal{T}_{h}\right)$, compute the new iterate $\mathbf{j}_{\mathbf{h}}^{(k+1)} \in \mathbb{R}^{n_{h}}$ by means of

$$
\mathbf{j}_{\mathbf{h}}^{(k+1)}=\mathbf{j}_{\mathbf{h}}^{(k+1 / 2)}+\boldsymbol{\psi}_{\mathbf{h}}^{(k+1 / 2)} .
$$

Within a multigrid approach with respect to a hierarchy $\mathcal{T}_{h_{j}}, 0 \leq j \leq L$, of nested simplicial triangulations of the computational domain $\Omega$, the hybrid iterative process is used as a smoother on all levels $1 \leq j \leq L$ as well as an iterative solver on the lowest level $j=0$. The convergence of multigrid V-cycles with the hybrid smoother and canonical intergrid transfers has been analyzed in [36] (cf. also [37]) in the framework of multiplicative Schwarz iterations with respect to a multilevel decomposition of the edge element space $\mathbf{N d}_{1, \Gamma}\left(\Omega ; \mathcal{T}_{L}\right)$ with respect to the finest grid.

For the algebraic formulation of the mortar edge element approximation (4.22) and the defect correction problem (4.39) we denote by $\mathbf{A}_{\mathbf{h}}$ the block matrix

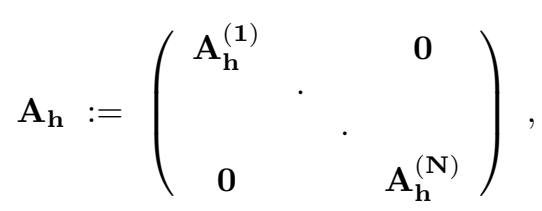

where $\mathbf{A}_{\mathbf{h}}^{(\mathbf{i})}, 1 \leq i \leq N$, are the matrix representations of the edge element discretized subdomain problems, and by $\mathbf{b}_{\mathbf{h}}=\left(\mathbf{b}_{\mathbf{h}}^{(\mathbf{1})}, \ldots, \mathbf{b}_{\mathbf{h}}^{(\mathbf{N})}\right)^{T}$ the matrix representation of $\ell_{h}(\cdot)$ restricted to $\mathbf{N d}_{1, \Gamma}\left(\Omega_{i} ; \mathcal{T}_{i}\right), 1 \leq i \leq N$. Moreover, we refer to $\mathbf{B}_{\mathbf{h}}$ as the matrix associated with the bilinear form $b_{h}(\cdot, \cdot)$. Then, the mortar edge element approximation $(4.22)$ can be written as the algebraic saddle point problem

$$
\left(\begin{array}{cc}
\mathbf{A}_{\mathbf{h}} & \mathbf{B}_{\mathbf{h}}^{\mathbf{T}} \\
\mathbf{B}_{\mathbf{h}} & \mathbf{0}
\end{array}\right)\left(\begin{array}{c}
\mathbf{j}_{\mathbf{h}} \\
\lambda_{\mathbf{h}}
\end{array}\right)=\left(\begin{array}{c}
\mathbf{b}_{\mathbf{h}} \\
\mathbf{0}
\end{array}\right) .
$$

Likewise, we denote by $\mathbf{C}_{\mathbf{h}}$ the block matrix

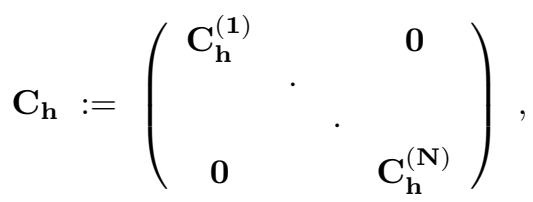

where $\mathbf{C}_{\mathbf{h}}^{(\mathbf{i})}, 1 \leq i \leq N$, are the matrix representations of $c_{h}(\cdot, \cdot)$ restricted to $S_{1, \Gamma}\left(\Omega_{i} ; \mathcal{T}_{i}\right) \times S_{1, \Gamma}\left(\Omega_{i} ; \mathcal{T}_{i}\right), 1 \leq i \leq N$, and by $\mathbf{r}_{\mathbf{h}}=\left(\mathbf{r}_{\mathbf{h}}^{(\mathbf{1})}, \ldots, \mathbf{r}_{\mathbf{h}}^{(\mathbf{N})}\right)^{T}$ the vector associated with $r_{h}(\cdot)$ restricted to $S_{1, \Gamma}\left(\Omega_{i} ; \mathcal{T}_{i}\right), 1 \leq i \leq N$. We further refer to $\mathbf{D}_{\mathbf{h}}$ as the matrix associated with the bilinear form $d_{h}(\cdot, \cdot)$. Then, the defect correction (4.39) can be as well written as an algebraic saddle point problem

$$
\left(\begin{array}{cc}
\mathbf{C}_{\mathbf{h}} & \mathbf{D}_{\mathbf{h}}^{\mathbf{T}} \\
\mathbf{D}_{\mathbf{h}} & \mathbf{0}
\end{array}\right)\left(\begin{array}{c}
\mathbf{u}_{\mathbf{h}} \\
\lambda_{\mathbf{h}}
\end{array}\right)=\left(\begin{array}{c}
\mathbf{r}_{\mathbf{h}} \\
\mathbf{0}
\end{array}\right)
$$

We are now in a position to state the hybrid iterative process with respect to the mortar edge element approximation of the eddy currents equations:

\section{Step 1: Iterative sweeps on the mortar edge element discretized problem}


Compute $\left(\mathbf{j}_{\mathbf{h}}^{(k+1 / 2)}, \boldsymbol{\lambda}_{\mathbf{h}}^{(k+1 / 2)}\right)$ by the application of $\mu_{1}>0$ preconditioned Richardson iterations to the saddle point problem (5.10)

$$
\begin{aligned}
& \left(\begin{array}{c}
\mathbf{j}_{\mathbf{h}}^{(j+1)} \\
\lambda_{\mathbf{h}}^{(j+1)}
\end{array}\right)=\left(\begin{array}{c}
\mathbf{j}_{\mathbf{h}}^{(j)} \\
\lambda_{\mathbf{h}}^{(j)}
\end{array}\right)- \\
& \left(\begin{array}{cc}
\mathbf{R}_{\mathbf{h}}^{(\mathbf{1})} & \mathbf{B}_{\mathbf{h}}^{\mathbf{T}} \\
\mathbf{B}_{\mathbf{h}} & \mathbf{0}
\end{array}\right)^{-\mathbf{1}}\left\{\left(\begin{array}{cc}
\mathbf{A}_{\mathbf{h}} & \mathbf{B}_{\mathbf{h}}^{\mathbf{T}} \\
\mathbf{B}_{\mathbf{h}} & \mathbf{0}
\end{array}\right)\left(\begin{array}{c}
\mathbf{j}_{\mathbf{h}}^{(j)} \\
\boldsymbol{\lambda}_{\mathbf{h}}^{(j)}
\end{array}\right)-\left(\begin{array}{c}
\mathbf{b}_{\mathbf{h}} \\
\mathbf{0}
\end{array}\right)\right\},
\end{aligned}
$$

where $\mathbf{R}_{\mathbf{h}}^{(\mathbf{1})}:=\operatorname{diag}\left(R_{h, 1}^{(1)}, \ldots, R_{h, N}^{(1)}\right)$ with $R_{h, i}^{(1)}, 1 \leq i \leq N$, represent damped Jacobi sweeps on the subdomain problems. We choose $\alpha_{1} \approx \lambda_{\max }\left(\mathbf{A}_{\mathbf{h}}\right)$ and $\left(\mathbf{j}_{\mathbf{h}}^{(0)}, \boldsymbol{\lambda}_{\mathbf{h}}^{(0)}\right)=$ $\left(\mathbf{j}_{\mathbf{h}}^{(\mathbf{k})}, \boldsymbol{\lambda}_{\mathbf{h}}^{(\mathbf{k})}\right)$ as startiterates.

\section{Step 2: Defect correction on the irrotational part}

Compute $\left(\mathbf{u}_{\mathbf{h}}^{(k+1 / 2)}, \boldsymbol{\eta}_{\mathbf{h}}^{(k+1 / 2)}\right)$ by the application of $\mu_{2}>0$ preconditioned Richardson iterations to the defect correction problem (5.11)

$$
\begin{aligned}
& \left(\begin{array}{c}
\mathbf{u}_{\mathbf{h}}^{(j+1)} \\
\boldsymbol{\eta}_{\mathbf{h}}^{(j+1)}
\end{array}\right)=\left(\begin{array}{c}
\mathbf{u}_{\mathbf{h}}^{(j)} \\
\boldsymbol{\eta}_{\mathbf{h}}^{(j)}
\end{array}\right)- \\
& \left(\begin{array}{cc}
\mathbf{R}_{\mathbf{h}}^{(2)} & \mathbf{D}_{\mathbf{h}}^{\mathbf{T}} \\
\mathbf{D}_{\mathbf{h}} & \mathbf{0}
\end{array}\right)^{-1}\left\{\left(\begin{array}{cc}
\mathbf{C}_{\mathbf{h}} & \mathbf{D}_{\mathbf{h}}^{\mathbf{T}} \\
\mathbf{D}_{\mathbf{h}} & \mathbf{0}
\end{array}\right)\left(\begin{array}{l}
\mathbf{u}_{\mathbf{h}}^{(j)} \\
\boldsymbol{\eta}_{\mathbf{h}}^{(j)}
\end{array}\right)-\left(\begin{array}{c}
\mathbf{r}_{\mathbf{h}}^{(\mathbf{k}+\mathbf{1} / \mathbf{2})} \\
\mathbf{0}
\end{array}\right)\right\}
\end{aligned}
$$

where $\mathbf{R}_{\mathbf{h}}^{(\mathbf{2})}:=\operatorname{diag}\left(R_{h, 1}^{(2)}, \ldots, R_{h, N}^{(2)}\right)$ with $R_{h, i}^{(2)}, 1 \leq i \leq N$, are damped Jacobi sweeps on the defect correction problems associated with the individual subdomains and $\mathbf{r}_{\mathbf{h}}^{(k+1 / 2)}$ is the vector representing $r_{h}(\cdot)$ with $\mathbf{j}_{\mathbf{h}}$ replaced by $\mathbf{j}_{\mathbf{h}}^{(k+1 / 2)}$. We choose $\alpha_{2} \approx \lambda_{\max }\left(\mathbf{C}_{\mathbf{h}}\right)$ and $\left(\mathbf{u}_{\mathbf{h}}^{(0)}, \boldsymbol{\eta}_{\mathbf{h}}^{(0)}\right)=\left(\mathbf{u}_{\mathbf{h}}^{(k)}, \boldsymbol{\eta}_{\mathbf{h}}^{(k)}\right)$ as startiterates.

\section{Step 3: Additive correction}

Denoting by $\mathbf{z}_{\mathbf{h}}^{(k+1 / 2)}$ the vector representing the projection of $\operatorname{grad} u_{h}^{(k+1 / 2)}$, $u_{h}^{(k+1 / 2)} \in V_{h}$ onto $\mathbf{V}_{\mathbf{h}}$, compute $\mathbf{j}_{\mathbf{h}}^{(\nu+1)}$ according to

$$
\mathbf{j}_{\mathbf{h}}^{(k+1)}:=\mathbf{j}_{\mathbf{h}}^{(k+1 / 2)}+\mathbf{z}_{\mathbf{h}}^{(k+1 / 2)} .
$$

and set $\boldsymbol{\lambda}_{\mathbf{h}}^{(k+1)}=\boldsymbol{\lambda}_{\mathbf{h}}^{(k+1 / 2)}, \boldsymbol{\eta}_{\mathbf{h}}^{(k+1)}=\boldsymbol{\eta}_{\mathbf{h}}^{(k+1 / 2)}$.

From a computational point of view, a significant simplification can be achieved by using in Steps 1 and 2 a nondiagonal preconditioner only for the unknowns associated with edges (Step 1) and grid points (Step 2) in the interior of the subdomains, whereas a diagonal preconditioner is used on the skeleton of the decomposition:

Splitting the unknowns $\mathbf{j}_{\mathbf{h}}$ and $\mathbf{u}_{\mathbf{h}}$ into those associated with the interior of the subdomains and those situated on the skeleton, the realization of the preconditioners requires the solution of block systems of the form

$$
\left(\begin{array}{ccc}
\tilde{\mathbf{R}}_{\mathbf{h}}^{(\mathbf{1})} & \mathbf{0} & \mathbf{0} \\
\mathbf{0} & \alpha_{1} \mathbf{I}_{\mathbf{h}}^{(S)} & \tilde{\mathbf{B}}_{\mathbf{h}}^{\mathbf{T}} \\
\mathbf{0} & \tilde{\mathbf{B}}_{\mathbf{h}} & \mathbf{0}
\end{array}\right)\left(\begin{array}{c}
\mathbf{j}_{\mathbf{h}}^{(\mathbf{I})} \\
\mathbf{j}_{\mathbf{h}}^{(\mathbf{S})} \\
\boldsymbol{\lambda}_{\mathbf{h}}
\end{array}\right)=\left(\begin{array}{c}
\mathbf{b}_{\mathbf{h}}^{(\mathbf{I})} \\
\mathbf{b}_{\mathbf{h}}^{(\mathbf{S})} \\
\mathbf{0}
\end{array}\right)
$$


and

$$
\left(\begin{array}{ccc}
\tilde{\mathbf{R}}_{\mathbf{h}}^{(\mathbf{2})} & \mathbf{0} & \mathbf{0} \\
\mathbf{0} & \alpha_{2} \mathbf{I}_{\mathbf{h}}^{(S)} & \tilde{\mathbf{D}}_{\mathbf{h}}^{\mathbf{T}} \\
\mathbf{0} & \tilde{\mathbf{D}}_{\mathbf{h}} & \mathbf{0}
\end{array}\right)\left(\begin{array}{c}
\mathbf{u}_{\mathbf{h}}^{(\mathbf{I})} \\
\mathbf{u}_{\mathbf{h}}^{(\mathbf{S})} \\
\boldsymbol{\eta}_{\mathbf{h}}
\end{array}\right)=\left(\begin{array}{c}
\mathbf{r}_{\mathbf{h}}^{(\mathbf{I})} \\
\mathbf{r}_{\mathbf{h}}^{(\mathbf{S})} \\
\mathbf{0}
\end{array}\right) .
$$

The solution of both (5.14) and (5.15) invoke Schur complement systems with the Schur complements

$$
\begin{aligned}
& \mathbf{S}_{\mathbf{h}}^{(\mathbf{1})}:=\boldsymbol{\alpha}_{1}^{-1} \tilde{\mathbf{B}}_{\mathbf{h}} \tilde{\mathbf{B}}_{\mathbf{h}}^{T}, \\
& \mathbf{S}_{\mathbf{h}}^{(\mathbf{2})}:=\boldsymbol{\alpha}_{2}^{-1} \tilde{\mathbf{D}}_{\mathbf{h}} \tilde{\mathbf{D}}_{\mathbf{h}}^{T} .
\end{aligned}
$$

THEOREM 5.1. There exist constants $C_{i}>0,1 \leq i \leq 2$, independent of the subdomain triangulations and the number of subdomains such that

$$
\begin{aligned}
& \kappa\left(\tilde{B}_{\mathbf{h}} \tilde{B}_{\mathbf{h}}^{T}\right) \leq C_{1}, \\
& \kappa\left(\tilde{D}_{\mathbf{h}} \tilde{D}_{\mathbf{h}}^{T}\right) \leq C_{2},
\end{aligned}
$$

where $\kappa$ stands for the spectral condition number.

Proof. The assertion can be deduced from the inf-sup conditions (4.28) and (4.42).

With the simplification given by (5.14) and (5.15) we will use the hybrid iterative process as a smoother within a multigrid framework assuming nested hierarchies $\left(\mathcal{T}_{i, \ell}\right)_{\ell=0}^{L}$ of simplicial triangulations of the subdomains $\Omega_{i}, 1 \leq i \leq N$. We may further assume $\tilde{\mathbf{R}}_{\mathbf{h}}^{(i)}$ to be spectrally equivalent to $\alpha_{i} \mathbf{I}_{\mathbf{h}}^{(\mathbf{I})}, 1 \leq i \leq 2$, so that (5.12) and (5.13) take the form

$$
\begin{aligned}
& \left(\begin{array}{c}
\mathbf{j}_{\mathbf{h}}^{(j+1)} \\
\boldsymbol{\lambda}_{\mathbf{h}}^{(j+1)}
\end{array}\right)=\left(\begin{array}{c}
\mathbf{j}_{\mathbf{h}}^{(j)} \\
\boldsymbol{\lambda}_{\mathbf{h}}^{(j)}
\end{array}\right)- \\
& \left(\begin{array}{cc}
\alpha_{\mathbf{1}} \mathbf{I}_{\mathbf{h}} & \mathbf{B}_{\mathbf{h}}^{\mathbf{T}} \\
\mathbf{B}_{\mathbf{h}} & \mathbf{0}
\end{array}\right)^{-1}\left\{\left(\begin{array}{cc}
\mathbf{A}_{\mathbf{h}} & \mathbf{B}_{\mathbf{h}}^{\mathbf{T}} \\
\mathbf{B}_{\mathbf{h}} & \mathbf{0}
\end{array}\right)\left(\begin{array}{l}
\mathbf{j}_{\mathbf{h}}^{(j)} \\
\boldsymbol{\lambda}_{\mathbf{h}}^{(j)}
\end{array}\right)-\left(\begin{array}{c}
\mathbf{b}_{\mathbf{h}} \\
\mathbf{0}
\end{array}\right)\right\}, \\
& \left(\begin{array}{l}
\mathbf{u}_{\mathbf{h}}^{(j+1)} \\
\boldsymbol{\eta}_{\mathbf{h}}^{(j+1)}
\end{array}\right)=\left(\begin{array}{l}
\mathbf{u}_{\mathbf{h}}^{(j)} \\
\boldsymbol{\eta}_{h}^{(j)}
\end{array}\right)- \\
& \left(\begin{array}{cc}
\alpha_{\mathbf{2}} \mathbf{I}_{\mathbf{h}} & \mathbf{D}_{\mathbf{h}}^{\mathbf{T}} \\
\mathbf{D}_{\mathbf{h}} & \mathbf{0}
\end{array}\right)^{-1}\left\{\left(\begin{array}{cc}
\mathbf{C}_{\mathbf{h}} & \mathbf{D}_{\mathbf{h}}^{\mathbf{T}} \\
\mathbf{D}_{\mathbf{h}} & \mathbf{0}
\end{array}\right)\left(\begin{array}{c}
\mathbf{u}_{\mathbf{h}}^{(j)} \\
\boldsymbol{\eta}_{\mathbf{h}}^{(j)}
\end{array}\right)-\left(\begin{array}{c}
\mathbf{r}_{\mathbf{h}} \\
\mathbf{0}
\end{array}\right)\right\} .
\end{aligned}
$$

Following [21] and applying classical multigrid convergence theory combined with duality arguments for nonconforming finite element approximations, under the regularity assumption $\mathbf{j} \in \mathbf{H}^{\mathbf{1}}(\mathbf{c u r l} ; \Omega)$ a smoothing and an approximation property can be established. These properties imply multigrid convergence of W-cycles with a convergence rate that is independent of the number of subdomains, the number of levels in the hierarchies of triangulations, and the granularities of the triangulations.

\section{Residual-type a posteriori error estimators}

In this section, we will establish a residual-type a posteriori error estimator $\eta$ for the mortar edge element approximation of the eddy currents problem and prove its efficiency and reliability. 
The estimator consists of element residuals and face residuals

$$
\begin{aligned}
\eta^{2}:= & \sum_{i=1}^{N}\left[\sum_{T \in \mathcal{T}_{i}} \sum_{\nu=1}^{2}\left(\eta_{T}^{(\nu)}\right)^{2}+\sum_{F \in \mathcal{F}_{h}^{i n t}\left(\Omega_{i}\right)}\left(\left(\eta_{F}^{(1)}\right)^{2}+\left(\eta_{F}^{(5)}\right)^{2}\right)\right] \\
& +\sum_{j=1}^{N} \sum_{F \in \mathcal{F}_{h}\left(\delta_{m(j)}\right)}\left[\sum_{\nu=2}^{4}\left(\eta_{F}^{(\nu)}\right)^{2}+\sum_{\nu=6}^{8}\left(\eta_{F}^{(\nu)}\right)^{2}\right] .
\end{aligned}
$$

In particular, for the element residuals $\eta_{T}^{(\nu)}, 1 \leq \nu \leq 2$, we have

$$
\begin{aligned}
\eta_{T}^{(1)} & :=h_{T}\left\|\mathbf{f}_{\mathbf{h}}-\operatorname{curl} \chi \mathbf{c u r l} \mathbf{j}_{\mathbf{h}}-\boldsymbol{\sigma} \mathbf{j}_{\mathbf{h}}\right\|_{0, T}, \\
\eta_{T}^{(2)} & :=h_{T}\left\|\operatorname{div}\left(\boldsymbol{\sigma} \mathbf{j}_{\mathbf{h}}\right)\right\|_{0, T},
\end{aligned}
$$

where $\mathbf{f}_{\mathbf{h}}$ is the integral mean of $\mathbf{f}$ on $T \in \mathcal{T}_{i}, 1 \leq i \leq N$.

On the other hand, the face residuals $\eta_{F}^{(\nu)}, 1 \leq \nu \leq 8$, are given by

(6.10) $\eta_{F}^{(7)}:=h_{F}^{1 / 2}\left\|\lambda_{h}-\left\{\mathbf{n} \cdot\left(\boldsymbol{\sigma} \mathbf{j}_{\mathbf{h}}\right)\right\}\right\|_{0, F}, F \in \mathcal{F}_{h}\left(\delta_{m(j)}\right)$,

(6.11) $\eta_{F}^{(8)}:=h_{F}^{-1 / 2}\left\|\left[u_{h}\right]\right\|_{0, F}, F \in \mathcal{F}_{h}\left(\delta_{m(j)}\right)$.

Furthermore, the error estimation involves the data oscillations

$$
\mathrm{osc}^{2}:=\mathrm{osc}_{1}^{2}+\mathrm{osc}_{2}^{2}
$$

where

$$
\operatorname{osc}_{1}^{2}:=\sum_{i=1}^{N} \sum_{T \in \mathcal{T}_{i}} \operatorname{osc}_{T}^{2} \quad, \quad \operatorname{osc}_{2}^{2}:=\sum_{F \in \mathcal{F}_{h}(\Omega)} \operatorname{osc}_{F}^{2},
$$

and $\operatorname{osc}_{T}, T \in \mathcal{T}_{i}, 1 \leq i \leq N$, and $\operatorname{osc}_{F}, F \in \mathcal{F}_{h}(\Omega)$, are given by

(6.14) $\operatorname{osc}_{T}:=h_{T}\left\|\mathbf{f}-\mathbf{f}_{\mathbf{h}}\right\|_{0, T} \quad, \quad \operatorname{osc}_{F}:=h_{F}^{1 / 2}\left\|\mathbf{n} \cdot\left(\mathbf{f}-\mathbf{f}_{\mathbf{h}}\right)\right\|_{0, F}$.

REMARK 1. The data oscillations osc $, T \in \mathcal{T}_{i}, 1 \leq i \leq N$, and osc $F, F \in$ $\mathcal{F}_{h}(\Omega)$, are of higher order, if the right-hand side $\mathbf{f}$ is sufficiently smooth.

The main results of this section states that up to data oscillations the estimator $\eta$ provides a lower and an upper bound for the global discretization errors

$$
\begin{aligned}
\mathbf{e}_{\mathbf{j}}:=\mathbf{j}-\mathbf{j}_{\mathbf{h}} & , \quad \mathbf{e}_{\boldsymbol{\lambda}}:=\boldsymbol{\lambda}-\boldsymbol{\lambda}_{\mathbf{h}}, \\
e_{u}:=u-u_{h} & , \quad e_{\boldsymbol{\lambda}}:=\lambda-\lambda_{h} .
\end{aligned}
$$

The first result establishes the reliability of the estimator.

THEOREM 6.1. There exist positive constants $\Gamma_{\nu}, 1 \leq \nu \leq 2$, depending only on the shape regularity of the triangulations $\mathcal{T}_{i}, 1 \leq i \leq N$, such that

$$
\left\|\mathbf{e}_{\mathbf{j}}^{\perp}\right\|_{\mathbf{V}}^{2}+\left\|e_{u}\right\|_{V}^{2}+\left\|\mathbf{e}_{\boldsymbol{\lambda}}^{\perp}\right\|_{\mathbf{M}(S)}^{2}+\left\|e_{\lambda}\right\|_{M(S)}^{2} \leq \Gamma_{1} \eta^{2}+\Gamma_{2} o s c^{2} .
$$


The second result shows the efficiency of the estimator.

THEOREM 6.2. There exist positive constants $\gamma_{\nu}, 1 \leq \nu \leq 2$, depending only on the shape regularity of the triangulations $\mathcal{T}_{i}, 1 \leq i \leq N$, such that

$$
\gamma_{1} \eta^{2}-\gamma_{2} o s c^{2} \leq\left\|\mathbf{e}_{\mathbf{j}}\right\|_{\mathbf{V}}^{2}+\|\| e_{u}\left\|_{V}^{2}+\right\| \mathbf{e}_{\boldsymbol{\lambda}}\left\|_{\mathbf{M}(S)}^{2}+\right\| e_{\lambda} \|_{M(S)}^{2} .
$$

6.1. Reliability of the error estimator. It is easy to see that the global discretization error $\left(\mathbf{e}_{\mathbf{j}}, \mathbf{e}_{\boldsymbol{\lambda}}\right) \in \mathbf{V} \times \mathbf{M}(S)$ satisfies the error equation

$$
\begin{array}{rlrl}
a\left(\mathbf{e}_{\mathbf{j}}, \mathbf{q}\right)+b\left(\mathbf{q}, \mathbf{e}_{\boldsymbol{\lambda}}\right) & =r_{1}^{(1)}(\mathbf{q}), & \mathbf{q} \in \mathbf{V}, \\
b\left(\mathbf{e}_{\mathbf{j}}, \boldsymbol{\mu}\right) & =r_{2}^{(1)}(\boldsymbol{\mu}), \quad \boldsymbol{\mu} \in \mathbf{M}(S) .
\end{array}
$$

Here, the residuals $r_{i}^{(1)}, 1 \leq i \leq 2$, are given by

$$
\begin{aligned}
& r_{1}^{(1)}(\mathbf{q}):=(\mathbf{f}, \mathbf{q})_{0, \Omega}-a\left(\mathbf{j}_{h}, \mathbf{q}\right)-b\left(\mathbf{q}, \boldsymbol{\lambda}_{\mathbf{h}}\right), \\
& r_{2}^{(1)}(\boldsymbol{\mu}):=-b\left(\mathbf{j}_{\mathbf{h}}, \boldsymbol{\mu}\right) .
\end{aligned}
$$

In view of (3.22) and (3.25), we decompose the errors $\mathbf{e}_{\mathbf{j}} \in \mathbf{V}$ and $\mathbf{e}_{\boldsymbol{\lambda}} \in \mathbf{M}(S)$ according to

$$
\begin{aligned}
\mathbf{e}_{\mathbf{j}}:=\mathbf{e}_{\mathbf{j}}^{0}+\mathbf{e}_{\mathbf{j}}^{\perp}, & \mathbf{e}_{\mathbf{j}}^{0} \in \mathbf{V}^{0}, \mathbf{e}_{\mathbf{j}}^{\perp} \in \mathbf{V}^{\perp}, \\
\mathbf{e}_{\boldsymbol{\lambda}}:=\mathbf{e}_{\boldsymbol{\lambda}}^{0}+\mathbf{e}_{\boldsymbol{\lambda}}^{\perp}, & \mathbf{e}_{\boldsymbol{\lambda}}^{0} \in \mathbf{M}^{0}(S), \mathbf{e}_{\boldsymbol{\lambda}}^{\perp} \in \mathbf{M}^{\perp}(S) .
\end{aligned}
$$

Then, it follows that the pair $\left(\mathbf{e}_{\mathbf{j}}^{\perp}, \mathbf{e}_{\boldsymbol{\lambda}}^{\perp}\right) \in \mathbf{V}^{\perp} \times \mathbf{M}^{\perp}(S)$ satisfies the saddle point problem

$$
\begin{aligned}
a\left(\mathbf{e}_{\mathbf{j}}^{\perp}, \mathbf{q}^{\perp}\right)+b\left(\mathbf{q}^{\perp}, \mathbf{e}_{\boldsymbol{\lambda}}^{\perp}\right) & =r_{1}^{(1)}\left(\mathbf{q}^{\perp}\right), \quad \mathbf{q}^{\perp} \in \mathbf{V}^{\perp}, \\
b\left(\mathbf{e}_{\mathbf{j}}^{\perp}, \boldsymbol{\mu}^{\perp}\right) & =r_{2}^{(1)}\left(\boldsymbol{\mu}^{\perp}\right), \quad \boldsymbol{\mu}^{\perp} \in \mathbf{M}^{\perp}(S) .
\end{aligned}
$$

We denote by $\mathbf{B}^{\perp}: \mathbf{V}^{\perp} \rightarrow \mathbf{M}^{\perp}(S)^{*}$ the operator associated with $\left.b(\cdot, \cdot)\right|_{\mathbf{V}^{\perp} \times \mathbf{M}^{\perp}(S)}$ in the sense that

$$
<\mathbf{B}^{\perp} \mathbf{q}^{\perp}, \boldsymbol{\mu}^{\perp}>=b_{1}\left(\mathbf{q}^{\perp}, \boldsymbol{\mu}^{\perp}\right) \quad, \quad \mathbf{q}^{\perp} \in \mathbf{V}^{\perp}, \boldsymbol{\mu}^{\perp} \in \mathbf{M}^{\perp}(S) .
$$

Then, we have

LEMMA 6.1. The bilinear form $\left.a(\cdot, \cdot)\right|_{\mathbf{V}^{\perp} \times \mathbf{V}^{\perp}}$ is elliptic on $\operatorname{Ker} \mathbf{B}^{\perp}=$ $\mathbf{H}_{0, \Gamma}\left(\mathbf{c u r l}^{\mathbf{0}} ; \Omega\right)$ and the bilinear form $\left.b(\cdot, \cdot)\right|_{\mathbf{V}^{\perp} \times \mathbf{M}^{\perp}(S)}$ satisfies the inf-sup condition

$$
\inf _{\boldsymbol{\mu}^{\perp} \in \mathbf{M}^{\perp}(S)} \sup _{\mathbf{q}^{\perp} \in \mathbf{V} \perp} \frac{b\left(\mathbf{q}^{\perp}, \boldsymbol{\mu}^{\perp}\right)}{\left\|\mathbf{q}^{\perp}\right\|_{\mathbf{V}}\left\|\boldsymbol{\mu}^{\perp}\right\|_{\mathbf{M}(S)}} \geq c>0 .
$$

PROOF. The proof of (6.23) can be established in the same way as the proof of (3.19) in Lemma 3.3.

COROLlary 6.1. The saddle point problem (6.22) admits a unique solution $\left(\mathbf{e}_{\mathbf{j}}^{\perp}, \mathbf{e}_{\boldsymbol{\lambda}}^{\perp}\right) \in \mathbf{V}^{\perp} \times \mathbf{M}^{\perp}(S)$ satisfying

$$
\left\|\mathbf{e}_{\mathbf{j}}^{\perp}\right\|_{\mathbf{V}}+\left\|\mathbf{e}_{\lambda}^{\perp}\right\|_{\mathbf{M}(S)} \leq C\left[\sup _{\mathbf{q}^{\perp} \in \mathbf{V}^{\perp}} \frac{\left|r_{1}^{(1)}\left(\mathbf{q}^{\perp}\right)\right|}{\left\|\mathbf{q}^{\perp}\right\|_{\mathbf{V}}}+\sup _{\boldsymbol{\mu}^{\perp} \in \mathbf{M}^{\perp}(S)} \frac{\left|r_{2}^{(1)}\left(\boldsymbol{\mu}^{\perp}\right)\right|}{\left\|\boldsymbol{\mu}^{\perp}\right\|_{\mathbf{M}(S)}}\right]
$$


In order to establish upper bounds for the two terms on the right-hand side in (6.24), we follow [9] and introduce vector-valued quasi-interpolation operators

$$
\begin{aligned}
& \mathbf{P}_{\mathbf{N d}, \mathbf{h}}^{(i)}: \mathbf{H}^{\mathbf{1}}\left(\Omega_{i}\right) \cap \mathbf{H}_{0, \Gamma}\left(\operatorname{curl} ; \Omega_{i}\right) \rightarrow \mathbf{N d}_{1,0}\left(\Omega_{i} ; \mathcal{T}_{i}\right) \\
& \mathbf{P}_{\mathbf{N d}, \mathbf{h}}^{(i)} \mathbf{q}^{(i)}:=\sum_{E \in \mathcal{E}_{h}\left(\Omega_{i}\right)} \int_{F_{E}} \gamma_{\mathbf{t}}\left(\mathbf{q}^{(i)}\right) \cdot \boldsymbol{\varphi}_{E}^{F} d \sigma \mathbf{q}_{\mathbf{N d}, E}^{(i)},
\end{aligned}
$$

where $F_{E} \in \mathcal{F}\left(\Omega_{i}\right)$ is the face opposite to $E$ and $\mathbf{q}_{\mathbf{N d}, E}^{(i)} \in \mathbf{N d}_{1,0}\left(\Omega_{i} ; \mathcal{T}_{h}^{(\nu)}\right)$ is the canonical basis function associated with the edge $E$. Moreover, denoting by $\mathbf{a}_{E^{\prime}}, E^{\prime} \in \mathcal{E}(F)$, the vertex of $F$ opposite to $E^{\prime}$, we refer to $\varphi_{E}^{F}, E \in \mathcal{E}(F)$ as the function given by

$$
\varphi_{E}^{F}(\mathbf{x}):= \pm \sum_{E^{\prime} \in \mathcal{E}(F)} a_{E^{\prime} E}\left(\mathbf{x}-\mathbf{a}_{E^{\prime}}\right) \quad, \quad \mathbf{x} \in \mathcal{T}_{i}
$$

with $a_{E^{\prime} E} \in \mathbb{R}$ chosen such that

$$
\int_{F}\left(\mathbf{q}_{E^{\prime}} \wedge \mathbf{n}\right) \cdot \varphi_{E}^{F}(\cdot) d \sigma=\delta_{E^{\prime} E} \quad, \quad E, E^{\prime} \in \mathcal{E}(F) .
$$

$\mathbf{P}_{\mathbf{N d}, \mathbf{h}}^{(i)}$ is a projection operator with $\mathbf{P}_{\mathbf{N d}, \mathbf{h}}^{(i)} \mathbf{q}^{(i)}=\mathbf{q}^{(i)}$ for $\mathbf{q}^{(i)} \in \mathbf{N d}_{1,0}\left(\Omega_{i} ; \mathcal{T}_{i}\right)$. Moreover, denoting by $D_{E}, E \in \mathcal{E}_{h}\left(\Omega_{i}\right), D_{F}, F \in \mathcal{F}_{h}\left(\Omega_{i}\right)$, and $D_{T}, T \in \mathcal{T}_{i}$, the sets

$$
\begin{aligned}
D_{E} & :=\bigcup\left\{T \in \mathcal{T}_{i} \mid E \in \mathcal{E}_{h}(T)\right\}, \\
D_{F} & :=\bigcup\left\{D_{E} \mid E \in \mathcal{E}_{h}(F)\right\}, \\
D_{T} & :=\bigcup\left\{D_{E} \mid E \in \mathcal{E}_{h}(T)\right\},
\end{aligned}
$$

we have the following stability and approximation properties of $\mathbf{P}_{\mathbf{N d}, \mathbf{h}}^{(i)}$.

LEMMA 6.2. There exists constants $d_{\nu}^{(i)}>0,1 \leq \nu \leq 4$, only depending on the shape regularity of $\mathcal{T}_{i}$, such that for $\mathbf{q} \in \mathbf{H}^{1}\left(\Omega_{i}\right)$ the operator $\mathbf{P}_{\mathbf{N d}, \mathbf{h}}^{(i)}$ satisfies

$$
\begin{aligned}
\left\|\mathbf{P}_{\mathbf{N d}, \mathbf{h}}^{(i)} \mathbf{q}\right\|_{0, T} & \leq d_{1}^{(i)}\|\mathbf{q}\|_{0, D_{T}} \\
\left\|\mathbf{c u r l} \mathbf{P}_{\mathbf{N d}, \mathbf{h}}^{(i)} \mathbf{q}\right\|_{0, T} & \leq d_{2}^{(i)}\|\mathbf{q}\|_{1, D_{F}} \\
\left\|\mathbf{q}-\mathbf{P}_{\mathbf{N d}, \mathbf{h}}^{(i)} \mathbf{q}\right\|_{0, T} & \leq d_{3}^{(i)} h_{T}\|\mathbf{q}\|_{1, D_{T}} \\
\left\|\mathbf{q}-\mathbf{P}_{\mathbf{N d}, \mathbf{h}}^{(i)} \mathbf{q}\right\|_{0, F} & \leq d_{4}^{(i)} h_{F}^{1 / 2}\|\mathbf{q}\|_{1, D_{F}}
\end{aligned}
$$

ProOF. We refer to [9].

By means of Lemmas 6.1 and 6.2 we are now able to provide an upper bound for the $\boldsymbol{\sigma}$-weakly solenoidal part $\mathbf{e}_{\mathbf{j}}^{\perp}$ of the error in $\mathbf{j}$ and the weakly irrotational part $\mathbf{e}_{\boldsymbol{\lambda}}^{\perp}$ of the error in the multiplier $\boldsymbol{\lambda}$. 
LEMMA 6.3. There exists constants $\Gamma_{\nu}>0,1 \leq \nu \leq 2$, depending only on the shape regularity of the triangulations $\mathcal{T}_{i}, 1 \leq i \leq N$, such that

$$
\begin{aligned}
(6.32)\left\|\mathbf{e}_{\mathbf{j}}^{\perp}\right\|_{\mathbf{V}}^{2}+\left\|\mathbf{e}_{\boldsymbol{\lambda}}^{\perp}\right\|_{\mathbf{M}(S)}^{2} \leq & \Gamma_{1}\left[\sum_{i=1}^{N}\left(\sum_{T \in \mathcal{T}_{i}}\left(\eta_{T}^{(1)}\right)^{2}+\sum_{F \in \mathcal{F}_{h}\left(\Omega_{i}\right)}\left(\eta_{F}^{(1)}\right)^{2}\right)\right. \\
& \left.+\sum_{F \in \mathcal{F}_{h}\left(\delta_{m(j)}\right)} \sum_{\nu=2}^{4}\left(\eta_{F}^{(\nu)}\right)^{2}\right]+\Gamma_{2} \operatorname{osc}_{1}^{2} .
\end{aligned}
$$

Proof. We first estimate $r_{1}^{(1)}\left(\mathbf{q}^{\perp}\right)$. Since $r_{1}^{(1)}\left(\mathbf{q}_{\mathbf{h}}^{\perp}\right)=0, \mathbf{q}_{h}^{\perp} \in \mathbf{V}_{\mathbf{h}}^{\perp}$, we have $\left.r_{1}^{(1)}\left(\mathbf{q}^{\perp}\right)=r_{1}^{(1)}\left(\mathbf{q}^{\perp}-\mathbf{q}_{\mathbf{h}}^{\perp}\right)\right)$. Choosing $\mathbf{q}_{\mathbf{h}}=\mathbf{P}_{\mathbf{N d}, \mathbf{h}}^{(i)} \mathbf{q}$, by element-wise and face-wise partial integration we obtain

$$
\begin{array}{r}
r_{1}^{(1)}\left(\mathbf{q}^{\perp}-\mathbf{q}_{\mathbf{h}}^{\perp}\right)=\sum_{i=1}^{N} \sum_{T \in \mathcal{T}_{i}}\left[\int_{T}\left(\mathbf{f}-\mathbf{f}_{\mathbf{h}}\right) \cdot\left(\mathbf{q}^{\perp}-\left(\mathbf{P}_{\mathbf{N d}, \mathbf{h}}^{(i)} \mathbf{q}\right)^{\perp}\right) d \mathbf{x}\right. \\
\left.+\int_{T}\left(\mathbf{f}_{\mathbf{h}}-\operatorname{curl} \chi \operatorname{curl} \mathbf{j}_{\mathbf{h}}-\boldsymbol{\sigma} \mathbf{j}_{\mathbf{h}}\right) \cdot\left(\mathbf{q}^{\perp}-\left(\mathbf{P}_{\mathbf{N d}, \mathbf{h}}^{(i)} \mathbf{q}\right)^{\perp}\right) d \mathbf{x}\right] \\
+\sum_{i=1}^{N} \sum_{F \in \mathcal{F}_{h}^{i n t}\left(\Omega_{i}\right)}<\left[\mathbf{n} \wedge\left(\chi \operatorname{curl} \mathbf{j}_{\mathbf{h}} \wedge \mathbf{n}\right)\right],\left(\mathbf{q}^{\perp}-\left(\mathbf{P}_{\mathbf{N d}, \mathbf{h}}^{(i)} \mathbf{q}\right)^{\perp}\right) \wedge \mathbf{n}>_{1 / 2, F} \\
+\sum_{i=1}^{N} \sum_{F \in \mathcal{F}_{h}\left(\delta_{m(j)}\right)}<\left[\mathbf{n} \wedge\left(\chi \mathbf{c u r l} \mathbf{j}_{\mathbf{h}} \wedge \mathbf{n}\right)\right],\left\{\left(\mathbf{q}^{\perp}-\left(\mathbf{P}_{\mathbf{N d}, \mathbf{h}}^{(i)} \mathbf{q}\right)^{\perp}\right) \wedge \mathbf{n}\right\}>_{1 / 2, F} \\
\sum_{i=1}^{N}<\boldsymbol{\lambda}_{F \in \mathcal{F}_{h}\left(\delta_{m(j)}\right)}-\left\{\mathbf{n} \wedge\left(\chi \mathbf{c u r l} \mathbf{j}_{\mathbf{h}} \wedge \mathbf{n}\right)\right\},\left[\left(\mathbf{q}^{\perp}-\left(\mathbf{P}_{\mathbf{N d}, \mathbf{h}}^{(i)} \mathbf{q}\right)^{\perp}\right) \wedge \mathbf{n}\right]>_{1 / 2, F}
\end{array}
$$

Using (6.29) in Lemma 6.2, for the volume integrals on the right-hand side of the previous equation we get

$$
\begin{aligned}
& \left|\left(\left(\mathbf{f}-\mathbf{f}_{\mathbf{h}}\right),\left(\mathbf{q}^{\perp}-\left(\mathbf{P}_{\mathbf{N d}, \mathbf{h}}^{(i)} \mathbf{q}\right)^{\perp}\right)\right)_{0, T}\right| \\
& \quad \leq d_{3}^{(i)} h_{T}\left\|\mathbf{f}-\mathbf{f}_{\mathbf{h}}\right\|_{0, T}\left\|\operatorname{curl} \mathbf{q}^{\perp}\right\|_{0, D_{T}} \\
& \left|\left(\left(\mathbf{f}_{\mathbf{h}}-\operatorname{curl} \chi \operatorname{curl} \mathbf{j}_{\mathbf{h}}-\boldsymbol{\sigma} \mathbf{j}_{\mathbf{h}}\right),\left(\mathbf{q}^{\perp}-\left(\mathbf{P}_{\mathbf{N d}, \mathbf{h}}^{(i)} \mathbf{q}\right)^{\perp}\right)\right)_{0, T}\right| \\
& \quad \leq d_{3}^{(i)} h_{T}\left\|\mathbf{f}_{\mathbf{h}}-\operatorname{curl} \chi \operatorname{curl} \mathbf{j}_{\mathbf{h}}-\boldsymbol{\sigma} \mathbf{j}_{\mathbf{h}}\right\|_{0, T}\left\|\mathbf{c u r l} \mathbf{q}^{\perp}\right\|_{0, D_{T}}
\end{aligned}
$$

On the other hand, using the inequality

$$
\left\|\boldsymbol{\mu}_{\mathbf{h}}\right\|_{-1 / 2, F} \leq C h_{F}^{1 / 2}
$$

where $\boldsymbol{\mu}_{\mathbf{h}} \in \mathbf{M}_{\mathbf{h}}(S)$ or $\boldsymbol{\mu}_{\mathbf{h}}=\left.\mathbf{q}_{\mathbf{h}}\right|_{F}, \mathbf{q}_{\mathbf{h}} \in \mathbf{V}_{\mathbf{h}}$, and the trace inequality

$$
\left\|\left.\mathbf{q}\right|_{F}\right\|_{1 / 2, F} \leq C\|\mathbf{q}\|_{1, T} \quad, \quad \mathbf{q} \in \mathbf{V}, F \in \mathcal{F}_{h}(T)
$$


as well as (6.28) and (6.29) in Lemma 6.2, for the third term on the right-hand side in (6.33) we obtain

$$
\begin{aligned}
(6.38) \mid & <\left[\mathbf{n} \wedge\left(\chi \operatorname{curl} \mathbf{j}_{\mathbf{h}} \wedge \mathbf{n}\right)\right],\left(\mathbf{q}^{\perp}-\left(\mathbf{P}_{\mathbf{N d}, \mathbf{h}}^{(i)} \mathbf{q}\right)^{\perp}\right) \wedge \mathbf{n}>_{1 / 2, F} \mid \\
& \leq\left\|\left[\mathbf{n} \wedge\left(\boldsymbol{\chi} \operatorname{curl} \mathbf{j}_{\mathbf{h}} \wedge \mathbf{n}\right)\right]\right\|_{-1 / 2, F}\left[\left\|\mathbf{q}^{\perp}\right\|_{1 / 2, F}+\left\|\left(\mathbf{P}_{\mathbf{N d}, \mathbf{h}}^{(i)} \mathbf{q}\right)^{\perp}\right\|_{1 / 2, F}\right] \\
& \leq C h_{F}^{1 / 2}\left\|\left[\mathbf{n} \wedge\left(\boldsymbol{\chi} \operatorname{curl} \mathbf{j}_{\mathbf{h}} \wedge \mathbf{n}\right)\right]\right\|_{0, F}\left[\left\|\mathbf{q}^{\perp}\right\|_{1, D_{F}^{2}}+\left\|\left(\mathbf{P}_{\mathbf{N d}, \mathbf{h}}^{(i)} \mathbf{q}\right)^{\perp}\right\|_{1, D_{F}}\right] \\
& \leq C h_{F}^{1 / 2}\left\|\left[\mathbf{n} \wedge\left(\boldsymbol{\chi} \operatorname{curl} \mathbf{j}_{\mathbf{h}} \wedge \mathbf{n}\right)\right]\right\|_{0, F}\left\|\mathbf{q}^{\perp}\right\|_{c u r l ; D_{F}}
\end{aligned}
$$

Likewise, the remaining two terms on the right-hand side in (6.33) can be estimated as follows

$$
\begin{aligned}
& \mid<\left[\mathbf{n} \wedge\left(\boldsymbol{\chi} \text { curl } \mathbf{j}_{\mathbf{h}} \wedge \mathbf{n}\right)\right],\left\{\left(\mathbf{q}^{\perp}-\left(\mathbf{P}_{\mathbf{N d}, \mathbf{h}}^{(i)} \mathbf{q}\right)^{\perp}\right) \wedge \mathbf{n}\right\}>_{1 / 2, F} \mid \\
& \quad \leq C h_{F}^{1 / 2}\left\|\left[\mathbf{n} \wedge\left(\boldsymbol{\chi} \mathbf{c u r l} \mathbf{j}_{\mathbf{h}} \wedge \mathbf{n}\right)\right]\right\|_{0, F}\left\|\mathbf{q}^{\perp}\right\|_{c u r l ; D_{F}^{2}} \\
& \left|<\boldsymbol{\lambda}_{\mathbf{h}}-\left\{\mathbf{n} \wedge\left(\boldsymbol{\chi} \mathbf{c u r l} \mathbf{j}_{\mathbf{h}} \wedge \mathbf{n}\right)\right\},\left[\left(\mathbf{q}^{\perp}-\left(\mathbf{P}_{\mathbf{N d}, \mathbf{h}}^{(i)} \mathbf{q}\right)^{\perp}\right) \wedge \mathbf{n}\right]>_{1 / 2, F}\right| \\
& \quad \leq C h_{F}^{1 / 2}\left\|\boldsymbol{\lambda}_{\mathbf{h}}-\left\{\mathbf{n} \wedge\left(\boldsymbol{\chi} \operatorname{curl} \mathbf{j}_{\mathbf{h}} \wedge \mathbf{n}\right)\right\}\right\|_{0, F}\left\|\mathbf{q}^{\perp}\right\|_{c u r l ; D_{F}} .
\end{aligned}
$$

There remains $r_{2}^{(1)}\left(\boldsymbol{\mu}^{\perp}\right)$ to be estimated.

$$
\left|r_{2}^{(1)}\left(\boldsymbol{\mu}^{\perp}\right)\right| \leq \sum_{i=1}^{N} \sum_{F \in \mathcal{F}_{h}\left(\delta_{m(j)}\right)}\left|<\boldsymbol{\mu}^{\perp},\left[\mathbf{j}_{\mathbf{h}} \wedge \mathbf{n}\right]>_{1 / 2, F}\right| .
$$

Using the inequality

$$
\left\|\left[\mathbf{j}_{\mathbf{h}} \wedge \mathbf{n}\right]\right\|_{1 / 2, F} \leq C h_{F}^{-1 / 2}\left\|\left[\mathbf{j}_{\mathbf{h}} \wedge \mathbf{n}\right]\right\|_{1 / 2, F}, \boldsymbol{\mu}^{\perp}>_{1 / 2, F} \mid .
$$

we arrive at

(6.43) $\left|<\boldsymbol{\mu}^{\perp},\left[\mathbf{j}_{\mathbf{h}} \wedge \mathbf{n}\right]>_{1 / 2, F}\right| \leq C h_{F}^{-1 / 2}\left\|\left[\mathbf{j}_{\mathbf{h}} \wedge \mathbf{n}\right]\right\|_{0, F}\left\|\boldsymbol{\mu}^{\perp}\right\|_{-1 / 2, F}$.

Summing over all $T \in \mathcal{T}_{i}$ and all $1 \leq i \leq N$, in (6.34),(6.35), over all $F \in \mathcal{F}_{h}^{\text {int }}\left(\Omega_{i}\right)$ and all $1 \leq i \leq N$, in (6.39), and over all $F \in \mathcal{F}_{h}\left(\delta_{m(j)}\right)$ in (6.40) as well as in (6.43), and inserting the results into $(6.33),(6.42)$, respectively, gives the assertion.

On the other hand, if $(u, \lambda) \in V \times M(S)$ is the solution of the macro-hybrid formulation (3.42) of the defect equation (3.32) for $u$ in the irrotational part $\mathbf{j}^{\mathbf{0}}=$ grad $u$ of the solution of $(3.1)$ and $\left(u_{h}, \lambda_{h}\right) \in V_{h} \times M_{h}(S)$ stands its mortar element approximation given by $(4.39)$, the error $\left(e_{u}, e_{\lambda}\right) \in V \times M(S)$ with

$$
e_{u}:=u-u_{h} \quad, \quad e_{\lambda}:=\lambda-\lambda_{h},
$$

satisfies

$$
\begin{array}{ll}
c\left(e_{u}, v\right)+d\left(v, e_{\lambda}\right) & =r_{1}^{(2)}(v), \quad v \in V, \\
d\left(e_{u}, \mu\right) & =r_{2}^{(2)}(\mu), \quad \mu \in M(S),
\end{array}
$$

where the residuals $r_{\nu}^{(2)}, 1 \leq \nu \leq 2$, are given by

$$
\begin{aligned}
r_{1}^{(2)}(v) & :=\sum_{i=1}^{N}\left(\mathbf{f}-\boldsymbol{\sigma} \mathbf{j}_{\mathbf{h}}, \operatorname{grad} v\right)_{0, \Omega_{i}}-d\left(v, \lambda_{h}\right), \\
r_{2}^{(2)}(\mu) & :=-d\left(u_{h}, \mu\right) .
\end{aligned}
$$


LEMMA 6.4. The saddle point problem (6.44) admits a unique solution $\left(e_{u}, e_{\lambda}\right) \in$ $V \times M(S)$ satisfying

(6.47) $\left\|e_{u}\right\|_{V}+\left\|e_{\lambda}\right\|_{M(S)} \leq C\left[\sup _{v \in V} \frac{\left|r_{1}^{(2)}(v)\right|}{\|v\|_{V}}+\sup _{\mu \in M(S)} \frac{\left|r_{2}^{(2)}(\mu)\right|}{\|\mu\|_{M(S)}}\right]$.

Proof. The proof is an immediate consequence of Theorem 3.2.

For the estimation of the two terms on the right-hand side in (6.47) we use the Scott-Zhang interpolation operators

$$
P_{S, h}^{(i)}: H_{0, \Gamma}^{1}\left(\Omega_{i}\right) \rightarrow S_{1,0}\left(\Omega_{i} ; \mathcal{T}_{i}\right)
$$

which have the following properties (cf. [60]):

LEMma 6.5. There exist constants $d_{\nu}^{(i)}, 5 \leq \nu \leq 8$, only depending on the shape regularity of $\mathcal{T}_{i}$, such that for $v \in H^{1}\left(\Omega_{i}\right)$ the operator $P_{S, h}^{(i)}$ satisfies

$$
\begin{aligned}
\left\|P_{S, h}^{(i)} v\right\|_{0, T} & \leq d_{6}^{(i)}\|v\|_{0, \tilde{D}_{T}} \quad, \quad T \in \mathcal{T}_{i}, \\
\left\|\operatorname{grad} P_{S, h}^{(i)} v\right\|_{0, T} & \leq d_{6}^{(i)}\|\operatorname{grad} v\|_{0, \tilde{D}_{T}}, \quad T \in \mathcal{T}_{i} \\
\left\|v-P_{S, h}^{(i)} v\right\|_{0, T} & \leq d_{7}^{(i)} h_{T}\|\operatorname{grad} v\|_{0, \tilde{D}_{T}} \quad, \quad T \in \mathcal{T}_{H}(\Omega), \\
\left\|v-P_{S, h}^{(i)} v\right\|_{0, F} & \leq d_{8}^{(i)} h_{F}^{1 / 2}\|\operatorname{grad} v\|_{0, \tilde{D}_{F}} \quad, \quad F \in \mathcal{F}_{h}\left(\Omega_{i}\right),
\end{aligned}
$$

with $\tilde{D}_{T}$ and $\tilde{D}_{E}$ being given by

$$
\begin{aligned}
& \tilde{D}_{T}:=\bigcup\left\{T^{\prime} \in \mathcal{T}_{i} \mid \mathcal{N}_{h}\left(T^{\prime}\right) \cap \mathcal{N}_{h}(T) \neq \emptyset\right\} \\
& \tilde{D}_{F}:=\bigcup\left\{T^{\prime} \in \mathcal{T}_{i} \mid \mathcal{N}_{h}\left(T^{\prime}\right) \cap \mathcal{N}_{h}(F) \neq \emptyset\right\}
\end{aligned}
$$

Lemma 6.4 and Lemma 6.5 enable us to establish an upper bound for the error $e_{u}$ of the error in $u$ and the error $e_{\lambda}$ in the multiplier $\lambda$.

LEMMA 6.6. There exist constants $\Gamma_{\nu}>0,5 \leq \nu \leq 6$, depending only on the shape regularity of the triangulations $\mathcal{T}_{i}, 1 \leq i \leq N$, such that

(6.55) $\left\|e_{u}\right\|_{V}^{2}+\left\|e_{\lambda}\right\|_{M(S)}^{2} \leq$

$$
\Gamma_{5}\left[\sum_{i=1}^{N}\left(\sum_{T \in \mathcal{T}_{i}}\left(\eta_{T}^{(2)}\right)^{2}+\sum_{F \in \mathcal{F}_{h}\left(\Omega_{i}\right)}\left(\eta_{F}^{(5)}\right)^{2}\right)+\sum_{F \in \mathcal{F}_{h}(S)} \sum_{\nu=6}^{8}\left(\eta_{F}^{(\nu)}\right)^{2}\right]+\Gamma_{6} \text { osc }_{2}^{2} .
$$

Proof. Observing $r_{1}^{(2)}\left(v_{h}\right)=0, v_{h} \in V_{h}$, we set $v_{h}:=P_{S, h}^{(i)} v$ and get by partial integration

$$
\begin{aligned}
r_{1}^{(2)}(v)= & r_{1}^{(2)}\left(v-P_{S, h}^{(i)} v\right) \\
= & \sum_{i=1}^{N} \sum_{T \in \mathcal{T}_{i}}\left[\int_{T}\left(\mathbf{f}-\mathbf{f}_{\mathbf{h}}\right) \cdot \operatorname{grad}\left(v-P_{S, h}^{(i)} v\right) d \mathbf{x}\right. \\
& \left.+\int_{T}\left(\mathbf{f}_{\mathbf{h}}-\boldsymbol{\sigma} \mathbf{j}_{\mathbf{h}}\right) \cdot \operatorname{grad}\left(v-P_{S, h}^{(i)} v\right) d \mathbf{x}\right]
\end{aligned}
$$




$$
\begin{aligned}
& +\sum_{m=1}^{M} \sum_{F \subset \delta_{m(j)}}<\lambda_{h},\left[v-P_{S, h}^{(i)} v\right]>_{1 / 2, F} \\
= & \sum_{i=1}^{N} \sum_{T \in \mathcal{T}_{i}} \int_{T} \operatorname{div} \boldsymbol{\sigma} \mathbf{j}_{\mathbf{h}}\left(v-P_{S, h}^{(i)} v\right) d \mathbf{x} \\
& +\sum_{F \in \mathcal{F}_{h}(\Omega)} \int_{F} \mathbf{n} \cdot\left(\mathbf{f}-\mathbf{f}_{\mathbf{h}}\right)\left(v-P_{S, h}^{(i)} v\right) d \boldsymbol{\sigma} \\
& -\sum_{i=1}^{N} \sum_{F \in \mathcal{F}_{h}\left(\Omega_{i}\right)}<\left[\mathbf{n} \cdot \boldsymbol{\sigma} \mathbf{j}_{\mathbf{h}}\right], v-P_{S, h}^{(i)} v>_{1 / 2, F} \\
& -\sum_{i=1}^{N} \sum_{F \in \mathcal{F}_{h}\left(\delta_{m(j)}\right)}<\left[\mathbf{n} \cdot \boldsymbol{\sigma} \mathbf{j}_{\mathbf{h}}\right],\left\{v-P_{S, h}^{(i)} v\right\}>_{1 / 2, F} \\
& +\sum_{i=1}^{N} \sum_{F \in \mathcal{F}_{h}\left(\delta_{m(j)}\right)}<\lambda_{h}-\left\{\mathbf{n} \cdot \boldsymbol{\sigma} \mathbf{j}_{\mathbf{h}}\right\},\left[v-P_{S, h}^{(i)} v\right]>_{1 / 2, F}
\end{aligned}
$$

By (6.51) and (6.52) in Lemma 6.5 we get

(6.57) $\quad\left|\left(\operatorname{div} \boldsymbol{\sigma} \mathbf{j}_{\mathbf{h}}, v-P_{S, h}^{(i)} v\right)_{0, T}\right|$

$$
\leq d_{7}^{(i)} h_{T}\left\|\operatorname{div} \boldsymbol{\sigma} \mathbf{j}_{\mathbf{h}}\right\|_{0, T}\|\operatorname{grad} v\|_{0, \tilde{D}_{T}} \quad, \quad T \in \mathcal{T}_{i},
$$

$$
\begin{aligned}
& \left|\left(\mathbf{n} \cdot\left(\mathbf{f}-\mathbf{f}_{\mathbf{h}}\right), v-P_{S, h}^{(i)} v\right)_{0, F}\right| \\
& \quad \leq d_{8}^{(i)} h_{F}^{1 / 2}\left\|\mathbf{n} \cdot\left(\mathbf{f}-\mathbf{f}_{\mathbf{h}}\right)\right\|_{0, F}\|\operatorname{grad} v\|_{0, \tilde{D}_{F}} \quad, \quad F \in \mathcal{F}_{h}(\Omega) .
\end{aligned}
$$

Moreover, using (6.37), (6.38) and (6.49), (6.50) in Lemma 6.5, we obtain

$$
\begin{aligned}
& \left|<\left[\mathbf{n} \cdot \boldsymbol{\sigma} \mathbf{j}_{\mathbf{h}}\right], v-P_{S, h}^{(i)} v>_{1 / 2, F}\right| \\
& \quad \leq\left\|\left[\mathbf{n} \cdot \boldsymbol{\sigma} \mathbf{j}_{\mathbf{h}}\right]\right\|_{-1 / 2, F}\left[\|v\|_{1 / 2, F}+\left\|P_{S, h}^{(i)} v\right\|_{1 / 2, F}\right] \\
& \quad \leq C h_{F}^{1 / 2}\left\|\left[\mathbf{n} \cdot \boldsymbol{\sigma} \mathbf{j}_{\mathbf{h}}\right]\right\|_{0, F}\|v\|_{1, \tilde{D}_{F}}, \quad F \in \mathcal{F}_{h}\left(\Omega_{i}\right), \\
& \left|<\left[\mathbf{n} \cdot \boldsymbol{\sigma} \mathbf{j}_{\mathbf{h}}\right],\left\{v-P_{S, h}^{(i)} v\right\}>_{1 / 2, F}\right| \\
& \quad \leq C h_{F}^{1 / 2}\left\|\left[\mathbf{n} \cdot \boldsymbol{\sigma} \mathbf{j}_{\mathbf{h}}\right]\right\|_{0, F}\|v\|_{1, \tilde{D}_{F}}, \quad F \in \mathcal{F}_{h}(S), \\
& \left|<\lambda_{h}-\left\{\mathbf{n} \cdot \boldsymbol{\sigma} \mathbf{j}_{\mathbf{h}}\right\},\left[v-P_{S, h}^{(i)} v\right]>_{1 / 2, F}\right| \\
& \quad \leq C h_{F}^{1 / 2}\left\|\left\{\mathbf{n} \cdot \boldsymbol{\sigma} \mathbf{j}_{\mathbf{h}}\right\}\right\|_{0, F}\|v\|_{1, \tilde{D}_{F}} \quad, \quad F \in \mathcal{F}_{h}\left(\delta_{m(j)}\right) .
\end{aligned}
$$

Finally, for $r_{2}^{(2)}(\mu)$ we get

$$
\left|r_{2}^{(2)}(\mu)\right| \leq \sum_{m=1}^{M} \sum_{F \in \delta_{m(j)}}\left|<\mu,\left[u_{h}\right]>_{-1 / 2, F}\right|
$$

Using (6.42), we find

$$
\left|<\mu,\left[u_{h}\right]>_{1 / 2, F}\right| \leq C h_{F}^{-1 / 2}\|\mu\|_{-1 / 2, F}\left\|\left[u_{h}\right]\right\|_{0, F} .
$$

for all $F \in \mathcal{F}_{h}\left(\delta_{m(j)}\right)$. 
Summing over all $T \in \mathcal{T}_{i}$ and all $1 \leq i \leq N$ in (6.57), over all $F \in \mathcal{F}_{h}^{\text {int }}(\Omega)$ in (6.58), over all $F \in \mathcal{F}_{h}\left(\Omega_{i}\right)$ and all $1 \leq i \leq N$ in (6.59) and over all $F \in \mathcal{F}_{h}\left(\delta_{m(j)}\right)$ in $(6.60),(6.61)$, and $(6.63)$, we conclude by inserting the results into (6.56) and (6.62), respectively.

Proof of Theorem 6.1. Using the upper bounds established in Lemmas 6.3 and 6.6 proves $(6.15)$.

6.2. Local efficiency of the error estimator. As far as the proof of the local efficiency of the error estimator $\eta$, as given by (6.1), is concerned, we use element bubble functions $\kappa_{T}, T \in \mathcal{T}_{i}, 1 \leq i \leq N$, and face bubble functions $\kappa_{F}, F \in$ $\mathcal{F}_{h}(\Omega)$, according to

$$
\kappa_{T}:=\prod_{\nu=1}^{4} \lambda_{\nu}^{(T)}, \quad \kappa_{F}:=\prod_{\nu=1}^{3} \lambda_{\nu}^{(F)},
$$

where $\lambda_{\nu}^{(T)}, 1 \leq \nu \leq 4$, and $\lambda_{\nu}^{(F)}, 1 \leq \nu \leq 3$, are the barycentric coordinates associated with the vertices $p_{\nu}^{(T)}, 1 \leq \nu \leq 4$, of $T$ and $p_{\nu}^{(F)}, 1 \leq \nu \leq 3$, of $F$. The following properties of $\kappa_{T}$ and $\kappa_{F}$ can be easily verified:

LEMMA 6.7. Let $\kappa_{T}$ and $\kappa_{F}$ be the element and face bubble functions defined by (6.64). Then, for $p_{h} \in P_{k}(T), k \in \mathbb{N}_{0}$, and $q_{h} \in P_{k}(F), k \in \mathbb{N}_{0}$, there holds:

$$
\begin{aligned}
&\left\|\kappa_{T}^{1 / 2} p_{h}\right\|_{0, T} \leq\left\|p_{h}\right\|_{0, T} \leq C\left\|\kappa_{T}^{1 / 2} p_{h}\right\|_{0, T}, \\
&\left\|\kappa_{F}^{1 / 2} p_{h}\right\|_{0, F} \leq\left\|p_{h}\right\|_{0, F} \leq C\left\|\kappa_{F}^{1 / 2} p_{h}\right\|_{0, T} .
\end{aligned}
$$

We will also frequently use the inverse inequalities

$$
\begin{array}{ll}
\left\|\operatorname{grad} p_{h}\right\|_{0, T} \leq h_{T}^{-1}\left\|p_{h}\right\|_{0, T} & , \quad p_{h} \in P_{k}(T), k \in \mathbb{N}, \\
\left\|\operatorname{curl} \mathbf{p}_{\mathbf{h}}\right\|_{0, T} \leq h_{T}^{-1}\left\|\mathbf{p}_{\mathbf{h}}\right\|_{0, T}, & \mathbf{p}_{\mathbf{h}} \in \mathbf{P}_{k}(T), k \in \mathbb{N},
\end{array}
$$

The proof of (6.16) in Theorem 6.2 is a direct consequence of the following local estimates:

Lemma 6.8. For $\eta_{T}^{(\nu)}, 1 \leq \nu \leq 2, T \in \mathcal{T}_{i}, 1 \leq i \leq N$, as given by (6.2) and (6.3) there holds

$$
\begin{aligned}
\eta_{T}^{(1)} & \leq C\left[\left\|\mathbf{e}_{\mathbf{j}}\right\|_{\text {curl }, T}+o s c_{T}\right] \\
\eta_{T}^{(2)} & \leq C\left\|\mathbf{e}_{\mathbf{j}}\right\|_{0, T} .
\end{aligned}
$$

Proof. For the proof of (6.69) we set $\mathbf{g}_{\mathbf{h}}:=\mathbf{f}_{\mathbf{h}}-\operatorname{curl} \chi \operatorname{curl} \mathbf{j}_{\mathbf{h}}-\boldsymbol{\sigma} \mathbf{j}_{\mathbf{h}}$. Observing (6.65), we obtain

$$
\begin{aligned}
& h_{T}^{-2}\left(\eta_{T}^{(1)}\right)^{2}=\left\|\mathbf{f}_{\mathbf{h}}-\operatorname{curl} \chi \operatorname{curl} \mathbf{j}_{\mathbf{h}}-\boldsymbol{\sigma} \mathbf{j}_{\mathbf{h}}\right\|_{0, T}^{2} \\
& \leq C \int_{T}\left(\mathbf{f}_{\mathbf{h}}-\operatorname{curl} \chi \operatorname{curl} \mathbf{j}_{\mathbf{h}}-\boldsymbol{\sigma} \mathbf{j}_{\mathbf{h}}\right) \cdot \kappa_{T} \mathbf{g}_{\mathbf{h}} d \mathbf{x} \\
& =C\left[\int_{T}\left(\mathbf{f}_{\mathbf{h}}-\mathbf{f}\right) \cdot \kappa_{T} \mathbf{g}_{\mathbf{h}} d \mathbf{x}+\int_{T}\left(\mathbf{f}-\operatorname{curl} \chi \operatorname{curl} \mathbf{j}_{\mathbf{h}}-\boldsymbol{\sigma} \mathbf{j}_{\mathbf{h}}\right) \cdot \kappa_{T} \mathbf{g}_{\mathbf{h}} d \mathbf{x}\right] .
\end{aligned}
$$

If we take $\left.\kappa_{T}\right|_{\partial T}=0$ into account, by Stokes' theorem

$$
\int_{T} \operatorname{curl} \chi \operatorname{curl} \mathbf{j}_{\mathbf{h}} \cdot \kappa_{T} \mathbf{g}_{\mathbf{h}} d \mathbf{x}=\int_{T} \chi \operatorname{curl} \mathbf{j}_{\mathbf{h}} \cdot \operatorname{curl}\left(\kappa_{T} \mathbf{g}_{\mathbf{h}}\right) d \mathbf{x} .
$$


On the other hand

$$
\int_{T} \mathbf{f} \cdot \kappa_{T} \mathbf{g}_{\mathbf{h}} d \mathbf{x}=\int_{T}\left[\chi \operatorname{curl} \mathbf{j} \cdot \operatorname{curl}\left(\kappa_{T} \mathbf{g}_{\mathbf{h}}\right)+\boldsymbol{\sigma} \mathbf{j} \cdot\left(\kappa_{T} \mathbf{g}_{\mathbf{h}}\right)\right] d \mathbf{x} .
$$

Consequently, using (6.65) and (6.68)

$$
\begin{aligned}
& \left\|\mathbf{f}_{\mathbf{h}}-\operatorname{curl} \chi \operatorname{curl} \mathbf{j}_{\mathbf{h}}-\boldsymbol{\sigma} \mathbf{j}_{\mathbf{h}}\right\|_{0, T}^{2} \\
\leq & C\left[\int_{T}\left(\mathbf{f}_{\mathbf{h}}-\mathbf{f}\right) \cdot\left(\kappa_{T} \mathbf{g}_{\mathbf{h}}\right) d \mathbf{x}+\int_{T}\left(\chi \operatorname{curl} \mathbf{e}_{\mathbf{j}}^{\perp} \cdot \operatorname{curl}\left(\kappa_{T} \mathbf{g}_{\mathbf{h}}\right)+\boldsymbol{\sigma} \mathbf{e}_{\mathbf{j}} \cdot\left(\kappa_{T} \mathbf{g}_{\mathbf{h}}\right)\right) d \mathbf{x}\right] \\
\leq & C\left[\left\|\operatorname{curl} \mathbf{e}_{\mathbf{j}}^{\perp}\right\|_{0, T}\left\|\operatorname{curl}\left(\kappa_{T} \mathbf{g}_{\mathbf{h}}\right)\right\|_{0, T}+\left\|\kappa_{T} \mathbf{g}_{\mathbf{h}}\right\|_{0, T}\left(\left\|\mathbf{e}_{\mathbf{j}}\right\|_{0, T}+\left\|\mathbf{f}-\mathbf{f}_{\mathbf{h}}\right\|_{0, T}\right)\right] \\
\leq & \left.C\left\|\mathbf{g}_{\mathbf{h}}\right\|_{0, T}\left[h_{T}^{-1}\left\|\operatorname{curl} \mathbf{e}_{\mathbf{j}}^{\perp}\right\|_{0, T}+\left\|\mathbf{e}_{\mathbf{j}}^{\mathbf{0}}\right\|_{0, T}+\left\|\mathbf{e}_{\mathbf{j}}^{\perp}\right\|_{0, T}+\left\|\mathbf{f}-\mathbf{f}_{\mathbf{h}}\right\|_{0, T}\right)\right] .
\end{aligned}
$$

Finally, in view of (6.71)

$$
\eta_{T}^{(1)} \leq C\left[\left\|\mathbf{e}_{\mathbf{j}}\right\|_{c u r l, T}+\operatorname{osc}_{T}\right]
$$

which proves (6.69).

In order to verify $(6.70)$, we set $g_{h}:=\operatorname{div}\left(\boldsymbol{\sigma} \mathbf{j}_{\mathbf{h}}\right)$. Using (6.65) and Green's formula, we obtain

$$
\begin{aligned}
h_{T}^{-2}\left(\eta_{T}^{(2)}\right)^{2} & =\left\|\operatorname{div} \boldsymbol{\sigma} \mathbf{j}_{\mathbf{h}}\right\|_{0, T}^{2} \\
& \leq C \int_{T}\left(\operatorname{div}\left(\boldsymbol{\sigma} \mathbf{j}_{\mathbf{h}}\right)^{2} \kappa_{T} d \mathbf{x}\right. \\
& =-C \int_{T} \boldsymbol{\sigma} \mathbf{j}_{\mathbf{h}} \cdot \operatorname{grad}\left(\kappa_{T} g_{h}\right) d \mathbf{x}
\end{aligned}
$$

On the other hand, since $\operatorname{div} \mathbf{f}=0$

$$
\begin{aligned}
\int_{T} \boldsymbol{\sigma} \mathbf{j} \cdot \operatorname{grad}\left(\kappa_{T} g_{h}\right) d \mathbf{x} & =\int_{T} \mathbf{f} \cdot \operatorname{grad}\left(\kappa_{T} g_{h}\right) d \mathbf{x} \\
& =-\int_{T} \kappa_{T} g_{h} \operatorname{div} d \mathbf{x} \\
& =0 .
\end{aligned}
$$

It follows that

$$
\begin{aligned}
\left\|\operatorname{div} \boldsymbol{\sigma} \mathbf{j}_{\mathbf{h}}\right\|_{0, T}^{2} & \leq C \int_{T} \boldsymbol{\sigma} \mathbf{e}_{\mathbf{j}} \cdot \operatorname{grad}\left(\kappa_{T} g_{h}\right) d \mathbf{x} \\
& \leq C\left\|\mathbf{e}_{\mathbf{j}}\right\|_{0, T}\left\|\operatorname{grad}\left(\kappa_{T} g_{h}\right)\right\|_{0, T} \\
& \leq C h_{T}^{-1}\left\|g_{h}\right\|_{0, T}\left\|\mathbf{e}_{\mathbf{j}}\right\|_{0, T} .
\end{aligned}
$$

Using (6.73) in (6.72) proves (6.70).

LEMMA 6.9. For $F \in \mathcal{F}_{h}\left(\Omega_{i}\right), 1 \leq i \leq N$, such that $F=T_{1} \cap T_{2}$ for some $T_{\nu} \in \mathcal{T}_{\nu}, 1 \leq \nu \leq 2$, let $\eta_{F}^{(1)}$ and $\eta_{F}^{(5)}$ be given by (6.4) and (6.8), respectively. Then, 
there holds

$$
\begin{aligned}
\eta_{F}^{(1)} & \leq C \sum_{\nu=1}^{2}\left(\left\|\mathbf{e}_{\mathbf{j}}\right\|_{c u r l, T_{\nu}}+\eta_{T_{\nu}}^{(1)}+\operatorname{osc}_{T_{\nu}}\right), \\
\eta_{F}^{(5)} & \leq C \sum_{\nu=1}^{2}\left[\left\|\mathbf{e}_{\mathbf{j}}\right\|_{0, T_{\nu}}+\eta_{T_{\nu}}^{(2)}\right) .
\end{aligned}
$$

ProOF. For the proof of $(6.74)$, we set $\mathbf{g}_{\mathbf{h}}:=\left[\mathbf{n} \wedge\left(\chi \mathbf{c u r l} \mathbf{j}_{\mathbf{h}} \wedge \mathbf{n}\right)\right]$ and define $\tilde{\mathbf{g}}_{\mathbf{h}} \in \mathbf{N d}_{1,0}\left(T_{1} \cup T_{2} ; \mathcal{T}_{i}\right)$ such that

$$
\left\|\tilde{\mathbf{g}}_{\mathbf{h}}\right\|_{0, T_{\nu}} \leq C h_{T_{\nu}}^{1 / 2}\left\|\mathbf{g}_{\mathbf{h}}\right\|_{0, F} \quad, \quad 1 \leq \nu \leq 2,
$$

and

$$
\begin{aligned}
h_{F}^{-1}\left(\eta_{F}^{(1)}\right)^{2} & =\left\|\left[\mathbf{n} \wedge\left(\chi \operatorname{curl} \mathbf{j}_{\mathbf{h}} \wedge \mathbf{n}\right)\right]\right\|_{0, F}^{2} \\
& \leq C \int_{F}\left[\mathbf{n} \wedge\left(\chi \operatorname{curl} \mathbf{j}_{\mathbf{h}} \wedge \mathbf{n}\right)\right] \cdot\left(\kappa_{F} \tilde{\mathbf{g}}_{\mathbf{h}} \wedge \mathbf{n}\right) d \boldsymbol{\sigma} .
\end{aligned}
$$

By Stokes' theorem

$$
\begin{aligned}
& \int_{F}\left[\mathbf{n} \wedge\left(\chi \operatorname{curl} \mathbf{j}_{\mathbf{h}} \wedge \mathbf{n}\right)\right] \cdot\left(\kappa_{F} \tilde{\mathbf{g}}_{\mathbf{h}} \wedge \mathbf{n}\right) d \boldsymbol{\sigma} \\
= & \sum_{\nu=1}^{2} \int_{T_{\nu}} \chi \operatorname{curl} \mathbf{j}_{\mathbf{h}} \cdot \operatorname{curl}\left(\kappa_{F} \tilde{\mathbf{g}}_{\mathbf{h}}\right) d \mathbf{x}-\sum_{\nu=1}^{2} \int_{T_{\nu}} \operatorname{curl} \chi \operatorname{curl} \mathbf{j}_{\mathbf{h}} \cdot\left(\kappa_{F} \tilde{\mathbf{g}}_{\mathbf{h}}\right) d \mathbf{x} .
\end{aligned}
$$

On the other hand

$$
\begin{aligned}
& \sum_{\nu=1}^{2} \int_{T_{\nu}}\left[\chi \operatorname{curl} \mathbf{j} \cdot \operatorname{curl}\left(\kappa_{F} \tilde{\mathbf{g}}_{\mathbf{h}}\right)+\boldsymbol{\sigma} \mathbf{j} \cdot\left(\kappa_{F} \tilde{\mathbf{g}}_{\mathbf{h}}\right)\right] d \mathbf{x} \\
= & \sum_{\nu=1}^{2} \int_{T_{\nu}} \mathbf{f} \cdot\left(\kappa_{F} \tilde{\mathbf{g}}_{\mathbf{h}}\right) d \mathbf{x} .
\end{aligned}
$$

Using (6.78), (6.79) in (6.77) and observing (6.68), (6.76), we get

$(6.80) \quad\left\|\left[\mathbf{n} \wedge\left(\chi \operatorname{curl} \mathbf{j}_{\mathbf{h}} \wedge \mathbf{n}\right)\right]\right\|_{0, F}^{2}$

$$
\begin{array}{r}
\leq C\left[\sum_{\nu=1}^{2} \int_{T_{\nu}}\left(\mathbf{f}-\mathbf{f}_{\mathbf{h}}\right) \cdot\left(\kappa_{F} \tilde{\mathbf{g}}_{\mathbf{h}}\right) d \mathbf{x}+\sum_{\nu=1}^{2} \int_{T_{\nu}}\left(\mathbf{f}_{\mathbf{h}}-\operatorname{curl} \chi \operatorname{curl} \mathbf{j}_{\mathbf{h}}-\boldsymbol{\sigma} \mathbf{j}_{\mathbf{h}}\right) \cdot\left(\kappa_{F} \tilde{\mathbf{g}}_{\mathbf{h}}\right) d \mathbf{x}\right. \\
\left.\quad-\sum_{\nu=1}^{2} \int_{T_{\nu}}\left(\chi \operatorname{curl} \mathbf{e}_{\mathbf{j}} \cdot \operatorname{curl}\left(\kappa_{F} \tilde{\mathbf{g}}_{\mathbf{h}}\right)+\boldsymbol{\sigma} \mathbf{e}_{\mathbf{j}} \cdot\left(\kappa_{F} \tilde{\mathbf{g}}_{\mathbf{h}}\right)\right) d \mathbf{x}\right] \\
\leq C\left\|\mathbf{g}_{\mathbf{h}}\right\|_{0, F} \sum_{\nu=1}^{2}\left[h_{T_{\nu}}^{1 / 2}\left(\left\|\mathbf{f}-\mathbf{f}_{\mathbf{h}}\right\|_{0, T_{\nu}}+\left\|\mathbf{f}_{h}-\operatorname{curl} \chi \operatorname{curl} \mathbf{j}_{\mathbf{h}}-\boldsymbol{\sigma} \mathbf{j}_{\mathbf{h}}\right\|_{0, T_{\nu}}\right)\right. \\
\left.+h_{T_{\nu}}^{-1 / 2}\left\|\operatorname{curl} \mathbf{e}_{\mathbf{j}}^{\perp}\right\|_{0, T_{\nu}}+h_{T_{\nu}}^{1 / 2}\left\|\mathbf{e}_{\mathbf{j}}\right\|_{0, T_{\nu}}\right] .
\end{array}
$$


Finally, in view of the shape regularity of the triangulations $\mathcal{T}_{i}, 1 \leq i \leq N$, we arrive at

$$
\eta_{F}^{(1)} \leq C \sum_{\nu=1}^{2}\left(\left\|\mathbf{e}_{\mathbf{j}}\right\|_{c u r l, T_{\nu}}+\eta_{T_{\nu}}^{(1)}+\operatorname{osc}_{T_{\nu}}\right),
$$

which is $(6.74)$.

For the proof of (6.75) we proceed in much the same way. We set $g_{h}:=\left[\mathbf{n} \cdot \boldsymbol{\sigma} \mathbf{j}_{\mathbf{h}}\right]$ and extend $g_{h}$ continuously to a polynomial function $\tilde{g}_{h}$ on $T_{1} \cup T_{2}$ such that

$$
\left\|\tilde{g}_{h}\right\|_{0, T_{\nu}} \leq C h_{T_{\nu}}^{1 / 2}\left\|g_{h}\right\|_{0, F} \quad, \quad 1 \leq \nu \leq 2 .
$$

Using again (6.3) and applying Green's theorem, it follows that

$$
\begin{aligned}
(6.82) h_{F}^{-1}\left(\eta_{F}^{(5)}\right)^{2} & =\left\|\left[\mathbf{n} \cdot \boldsymbol{\sigma} \mathbf{j}_{\mathbf{h}}\right]\right\|_{0, F}^{2} \\
& \leq C \int_{F}\left[\mathbf{n} \cdot \boldsymbol{\sigma} \mathbf{j}_{\mathbf{h}}\right] \kappa_{F} g_{h} d \boldsymbol{\sigma} \\
& =C \sum_{\nu=1}^{2} \int_{T_{\nu}}\left(\operatorname{div}\left(\boldsymbol{\sigma} \mathbf{j}_{\mathbf{h}}\right)\left(\kappa_{F} g_{h}\right)+\boldsymbol{\sigma} \mathbf{j}_{\mathbf{h}} \cdot \mathbf{g r a d}\left(\kappa_{F} g_{h}\right)\right) d \mathbf{x} .
\end{aligned}
$$

Taking $\operatorname{div} \mathbf{f}=0$ into account, we obtain

$$
\sum_{\nu=1}^{2} \int_{T_{\nu}} \boldsymbol{\sigma} \mathbf{j} \cdot \operatorname{grad}\left(\kappa_{F} g_{h}\right) d \mathbf{x}=0
$$

If we take advantage of (6.83) in (6.82) and observe (6.66), (6.67) and (6.81), we end up with

$$
\begin{aligned}
\left\|\left[\mathbf{n} \cdot \boldsymbol{\sigma} \mathbf{j}_{\mathbf{h}}\right]\right\|_{0, F}^{2} & \leq C \sum_{\nu=1}^{2} \int_{T_{\nu}}\left(\operatorname{div}\left(\boldsymbol{\sigma} \mathbf{j}_{\mathbf{h}}\right)\left(\kappa_{F} g_{h}\right)-\boldsymbol{\sigma} \mathbf{e}_{\mathbf{j}} \cdot \operatorname{grad}\left(\kappa_{F} g_{h}\right)\right) d \mathbf{x} \\
& \leq C\left\|g_{h}\right\|_{0, F} \sum_{\nu=1}^{2}\left(h_{T_{\nu}}^{1 / 2}\left\|\operatorname{div} \boldsymbol{\sigma} \mathbf{j}_{\mathbf{h}}\right\|_{0, T_{\nu}}+h_{T_{\nu}}^{-1 / 2}\left\|\mathbf{e}_{\mathbf{j}}\right\|_{0, T_{\nu}}\right),
\end{aligned}
$$

and hence,

$$
\eta_{F}^{(5)} \leq C \sum_{\nu=1}^{2}\left(\left\|\mathbf{e}_{\mathbf{j}}\right\|_{0, T_{\nu}}+\eta_{T_{\nu}}^{2}\right)
$$

which is $(6.75)$.

Lemma 6.10. Assume $F \in \mathcal{F}_{h}\left(\delta_{m(j)}\right)$ such that $F=T \cap \delta_{m(j)}$ for some $T \in \mathcal{T}_{j}$ and let $\eta_{F}^{(4)}$ and $\eta_{F}^{(8)}$ be given by (6.4) and (6.11), respectively. Then, there holds

$$
\begin{aligned}
& \eta_{F}^{(4)} \leq C\left\|\left[\mathbf{e}_{\mathbf{j}}\right] \wedge \mathbf{n}\right\|_{-1 / 2, h, F}, \\
& \eta_{F}^{(8)} \leq C\left\|\left[e_{u}\right]\right\|_{-1 / 2, h, F} .
\end{aligned}
$$

Proof. For the proof of (6.84) we set $\mathbf{g}_{\mathbf{h}}:=\left[\mathbf{j}_{\mathbf{h}} \wedge \mathbf{n}\right]$. Then, in view of $(6.66)$, we have

$$
h_{F}\left(\eta_{F}^{(4)}\right)^{2}=\left\|\left[\mathbf{j}_{\mathbf{h}} \wedge \mathbf{n}\right]\right\|_{0, F}^{2} \leq C \int_{F}\left[\mathbf{j}_{\mathbf{h}} \wedge \mathbf{n}\right] \cdot \kappa_{F} \mathbf{g}_{\mathbf{h}} d \boldsymbol{\sigma}
$$


On the other hand

$$
b\left(\mathbf{j}, \kappa_{F} \mathbf{g}_{\mathbf{h}}\right)=\int_{F}[\mathbf{j} \wedge \mathbf{n}] \cdot\left(\kappa_{F} \mathbf{g}_{\mathbf{h}}\right) d \boldsymbol{\sigma}=0 .
$$

Hence, taking (6.66) into account, (6.86) implies

$\left\|\left[\mathbf{j}_{\mathbf{h}} \wedge \mathbf{n}\right]\right\|_{0, F}^{2} \leq C \int_{F}\left[\left(\mathbf{j}_{\mathbf{h}}-\mathbf{j}\right) \wedge \mathbf{n}\right] \cdot\left(\kappa_{F} \mathbf{g}_{\mathbf{h}}\right) d \boldsymbol{\sigma} \leq C\left\|\mathbf{g}_{\mathbf{h}}\right\|_{0, F}\left\|\left[\mathbf{e}_{\mathbf{j}} \wedge \mathbf{n}\right]\right\|_{0, F}$,

whence

$$
\eta_{F}^{(4)} \leq C h_{F}^{-1 / 2}\left\|\left[\mathbf{e}_{\mathbf{j}} \wedge \mathbf{n}\right]\right\|_{0, F}=C\left\|\left[\mathbf{e}_{\mathbf{j}} \wedge \mathbf{n}\right]\right\|_{-1 / 2, h, F},
$$

which is (6.84).

The proof of (6.85) follows the same lines.

LEMMA 6.11. Assume $F_{\gamma} \in \mathcal{F}_{h}\left(\gamma_{m(i)}\right), F_{\delta} \in \mathcal{F}_{h}\left(\delta_{m(j)}\right)$ and $T_{F_{\gamma}} \in \mathcal{T}_{i}, T_{F_{\delta}} \in \mathcal{T}_{j}$ such that $F_{\gamma}=T_{F_{\gamma}} \cap \gamma_{m(i)}$ and $F_{\delta}=T_{F_{\delta}} \cap \delta_{m(j)}$. Let $\eta_{F_{\delta}}^{(\nu)}, 2 \leq \nu \leq 3$, be given by (6.5) and (6.6), respectively. Then, there holds

$$
\begin{aligned}
& \left(\sum_{F_{\delta} \in \mathcal{F}_{h}\left(\delta_{m(j)}\right)}\left(\left(\eta_{F_{\delta}}^{(2)}\right)^{2}+\left(\eta_{F_{\delta}}^{(3)}\right)^{2}\right)\right)^{1 / 2} \\
& \leq C\left[\sum_{F_{\delta} \in \mathcal{F}_{h}\left(\delta_{m(j)}\right)}\left(\left\|\mathbf{e}_{\mathbf{j}}\right\|_{c u r l, T_{F_{\delta}}}+\eta_{T_{F_{\delta}}}^{(1)}+o s c_{T_{F_{\delta}}}\right)\right. \\
& \left.\quad+\sum_{F_{\gamma} \in \mathcal{F}_{h}\left(\gamma_{m(i)}\right)}\left(\left\|\mathbf{e}_{\mathbf{j}}\right\|_{c u r l, T_{F_{\gamma}}}+\eta_{T_{F_{\gamma}}}^{(1)}+\operatorname{osc}_{T_{F_{\gamma}}}\right)+\left\|\mathbf{e}_{\boldsymbol{\lambda}}\right\|_{\mathbf{M}\left(\Gamma_{i j}\right)}\right] .
\end{aligned}
$$

ProOF. We set

$$
\begin{aligned}
& \mathbf{g}_{\mathbf{h}}^{(1)}:=\lambda_{\mathbf{h}}-\left\{\mathbf{n} \wedge\left(\chi \operatorname{curl} \mathbf{j}_{\mathbf{h}} \wedge \mathbf{n}\right)\right\} \\
& \mathbf{g}_{\mathbf{h}}^{(2)}:=\left[\mathbf{n} \wedge\left(\chi \operatorname{curl} \mathbf{j}_{\mathbf{h}} \wedge \mathbf{n}\right)\right]
\end{aligned}
$$

and construct

$$
\mathbf{p}_{\mathbf{h}}^{(1)} \in \mathbf{N d}_{1, \partial T_{F_{\gamma}} \backslash F_{\gamma}}\left(T_{F_{\gamma}}, \mathcal{T}_{i}\right) \quad, \quad \mathbf{p}_{\mathbf{h}}^{(2)} \in \mathbf{N d}_{1, \partial T_{F_{\delta}} \backslash F_{\delta}}\left(T_{F_{\delta}}, \mathcal{T}_{j}\right)
$$

such that

$$
\begin{aligned}
& \text { (6.88) }\left\|\kappa_{F_{\gamma}} \mathbf{p}_{\mathbf{h}}^{(1)} \wedge \mathbf{n}_{F_{\gamma}}\right\|_{0, F_{\gamma}} \leq C\left(\sum_{F_{\delta} \in \mathcal{F}_{h}\left(\delta_{m(j)}\right)}\left(\left\|\mathbf{g}_{\mathbf{h}}^{(1)}\right\|_{0, F_{\delta}}^{2}+\left\|\mathbf{g}_{\mathbf{h}}^{(2)}\right\|_{0, F_{\delta}}^{2}\right)\right)^{1 / 2}, \\
& (6.89)\left\|\kappa_{F_{\delta}} \mathbf{p}_{\mathbf{h}}^{(2)} \wedge \mathbf{n}_{F_{\delta}}\right\|_{0, F_{\delta}} \leq C\left(\sum_{F_{\delta} \in \mathcal{F}_{h}\left(\delta_{m(j)}\right)}\left(\left\|\mathbf{g}_{\mathbf{h}}^{(1)}\right\|_{0, F_{\delta}}^{2}+\left\|\mathbf{g}_{\mathbf{h}}^{(2)}\right\|_{0, F_{\delta}}^{2}\right)\right)^{1 / 2},
\end{aligned}
$$

$$
\left(\sum_{F_{\gamma} \in \mathcal{F}_{h}\left(\gamma_{m(i)}\right)}\left\|\mathbf{p}_{\mathbf{h}}^{(1)}\right\|_{0, T_{F_{\gamma}}}^{2}\right)^{1 / 2} \leq C h_{\gamma}^{1 / 2}\left(\sum_{F_{\delta} \in \mathcal{F}_{h}\left(\delta_{m(j)}\right)}\left(\left\|\mathbf{g}_{\mathbf{h}}^{(1)}\right\|_{0, F_{\delta}}^{2}+\left\|\mathbf{g}_{\mathbf{h}}^{(2)}\right\|_{0, F_{\delta}}^{2}\right)\right)^{1 / 2},
$$

$$
\left(\sum_{F_{\delta} \in \mathcal{F}_{h}\left(\delta_{m(j)}\right)}\left\|\mathbf{p}_{\mathbf{h}}^{(2)}\right\|_{0, T_{F_{\delta}}}^{2}\right)^{1 / 2} \leq C h_{\delta}^{1 / 2}\left(\sum_{F_{\delta} \in \mathcal{F}_{h}\left(\delta_{m(j)}\right)}\left(\left\|\mathbf{g}_{\mathbf{h}}^{(1)}\right\|_{0, F_{\delta}}^{2}+\left\|\mathbf{g}_{\mathbf{h}}^{(2)}\right\|_{0, F_{\delta}}^{2}\right)\right)^{1 / 2}
$$


and

$$
\begin{aligned}
\text { (6.92) } & \sum_{F_{\delta} \in \mathcal{F}_{h}\left(\delta_{m(j)}\right)} h_{F_{\delta}}^{-1}\left(\left(\eta_{F_{\delta}}^{(2)}\right)^{2}+\left(\eta_{F_{\delta}}^{(3)}\right)^{2}\right) \\
= & \sum_{F_{\delta} \in \mathcal{F}_{h}\left(\delta_{m(j)}\right)}\left(\left\|\boldsymbol{\lambda}_{\mathbf{h}}-\left\{\mathbf{n} \wedge\left(\chi \mathbf{c u r l} \mathbf{j}_{\mathbf{h}} \wedge \mathbf{n}\right)\right\}\right\|_{0, F_{\delta}}^{2}\right. \\
& \left.+\left\|\left[\mathbf{n} \wedge\left(\chi \mathbf{c u r l} \mathbf{j}_{\mathbf{h}} \wedge \mathbf{n}\right)\right]\right\|_{0, F_{\delta}}^{2}\right) \\
\leq & C\left[\left.\sum_{F_{\delta} \in \mathcal{F}_{h}\left(\delta_{m(j)}\right)} \int_{F_{\delta}} \boldsymbol{\lambda}_{\mathbf{h}} \cdot\left(\kappa_{F_{\delta}} \mathbf{p}_{\mathbf{h}}^{(2)} \wedge \mathbf{n}_{F_{\delta}}\right)\right|_{\delta_{m(j)} \cap F_{\delta}} d \boldsymbol{\sigma}\right. \\
& +\left.\sum_{F_{\gamma} \in \mathcal{F}_{h}\left(\gamma_{m(i)}\right)} \int_{F_{\gamma}} \boldsymbol{\lambda}_{\mathbf{h}} \cdot\left(\kappa_{F_{\gamma}} \mathbf{p}_{\mathbf{h}}^{(1)} \wedge \mathbf{n}_{F_{\gamma}}\right)\right|_{\gamma_{m(i)} \cap F_{\gamma}} d \boldsymbol{\sigma} \\
+ & \left.\sum_{F_{\delta} \in \mathcal{F}_{h}\left(\delta_{m(j)}\right)} \int_{F_{\delta}}\left(\mathbf{n}_{F_{\delta}} \wedge\left(\chi \mathbf{c u r l} \mathbf{j}_{\mathbf{h}} \wedge \mathbf{n}_{F_{\delta}}\right)\right)\right|_{\delta_{m(j)} \cap F_{\delta}} \cdot\left(\kappa_{F_{\delta}} \mathbf{p}_{\mathbf{h}}^{(2)} \wedge \mathbf{n}_{F_{\delta}}\right) d \boldsymbol{\sigma} \\
& \left.+\left.\sum_{F_{\gamma} \in \mathcal{F}_{h}\left(\gamma_{m(i)}\right)} \int_{F_{\gamma}}\left(\mathbf{n}_{F_{\gamma}} \wedge\left(\chi \operatorname{curl} \mathbf{j}_{\mathbf{h}} \wedge \mathbf{n}_{F_{\gamma}}\right)\right)\right|_{\gamma_{m(i)} \cap F_{\gamma}} \cdot\left(\kappa_{F_{\gamma}} \mathbf{p}_{\mathbf{h}}^{(1)} \wedge \mathbf{n}_{F_{\gamma}}\right) d \boldsymbol{\sigma}\right] .
\end{aligned}
$$

where in $(6.90),(6.91)$

$$
h_{\gamma}: \max _{T_{F_{\gamma}}} h_{T_{F_{\gamma}}}, \quad h_{\delta}: \max _{T_{F_{\delta}}} h_{T_{F_{\delta}}} .
$$

Applying Stokes' theorem to the integrals occurring in the third and fourth terms on the right-hand side in (6.29), we obtain

$$
\begin{aligned}
& \left.\int_{F_{\delta}}\left(\mathbf{n}_{F_{\delta}} \wedge\left(\chi \operatorname{curl} \mathbf{j}_{\mathbf{h}} \wedge \mathbf{n}_{F_{\delta}}\right)\right)\right|_{\delta_{m(j)} \cap F} \cdot\left[\kappa_{F_{\delta}} \mathbf{p}_{\mathbf{h}}^{(2)} \wedge \mathbf{n}_{F_{\delta}}\right] d \boldsymbol{\sigma} \\
= & \int_{T_{F_{\delta}}} \chi \operatorname{curl} \mathbf{j}_{\mathbf{h}} \cdot \operatorname{curl}\left(\kappa_{F_{\delta}} \mathbf{p}_{\mathbf{h}}^{(2)}\right) d \mathbf{x}-\int_{T_{F_{\delta}}} \operatorname{curl} \chi \operatorname{curl} \mathbf{j}_{\mathbf{h}} \cdot\left(\kappa_{F_{\delta}} \mathbf{p}_{\mathbf{h}}^{(2)}\right) d \mathbf{x} .
\end{aligned}
$$

and

$$
\begin{aligned}
& \left.\int_{F_{\gamma}}\left(\mathbf{n}_{F_{\gamma}} \wedge\left(\chi \operatorname{curl} \mathbf{j}_{\mathbf{h}} \wedge \mathbf{n}_{F_{\gamma}}\right)\right)\right|_{\gamma_{m(i)} \cap F_{\gamma}} \cdot\left[\kappa_{F_{\gamma}} \mathbf{p}_{\mathbf{h}}^{(1)} \wedge \mathbf{n}_{F_{\gamma}}\right] d \boldsymbol{\sigma} \\
= & \int_{T_{F_{\gamma}}} \chi \operatorname{curl} \mathbf{j}_{\mathbf{h}} \cdot \operatorname{curl}\left(\kappa_{F_{\gamma}} \mathbf{p}_{\mathbf{h}}^{(1)}\right) d \mathbf{x}-\int_{T_{F_{\gamma}}} \operatorname{curl} \chi \operatorname{curl} \mathbf{j}_{\mathbf{h}} \cdot\left(\kappa_{F_{\gamma}} \mathbf{p}_{\mathbf{h}}^{(1)}\right) d \mathbf{x} .
\end{aligned}
$$

On the other hand

$$
\begin{aligned}
& \int_{T_{F_{\delta}}}(\chi \operatorname{curl} \mathbf{j} \cdot \operatorname{curl}\left.\left(\kappa_{F_{\delta}} \mathbf{p}_{\mathbf{h}}^{(2)}\right)+\boldsymbol{\sigma} \mathbf{j} \cdot\left(\kappa_{F_{\delta}} \mathbf{p}_{\mathbf{h}}^{(2)}\right)\right) d \mathbf{x} \\
&+\int_{F_{\delta}} \boldsymbol{\lambda} \cdot\left(\kappa_{F_{\delta}} \mathbf{p}_{\mathbf{h}}^{(2)} \wedge \mathbf{n}_{F_{\delta}}\right) d \boldsymbol{\sigma}=\int_{T_{F_{\gamma}}} \mathbf{f} \cdot\left(\kappa_{F_{\delta}} \mathbf{p}_{\mathbf{h}}^{(2)}\right) d \mathbf{x}
\end{aligned}
$$


and

$(6.96)$

$$
\begin{aligned}
\int_{T_{F_{\gamma}}}\left(\chi \operatorname{curl} \mathbf{j} \cdot \operatorname{curl}\left(\kappa_{F_{\gamma}} \mathbf{p}_{\mathbf{h}}^{(1)}\right)+\boldsymbol{\sigma} \mathbf{j} \cdot\left(\kappa_{F_{\gamma}} \mathbf{p}_{\mathbf{h}}^{(1)}\right)\right) d \mathbf{x}+ \\
+\int_{F_{\gamma}} \boldsymbol{\lambda} \cdot\left(\kappa_{F_{\gamma}} \mathbf{p}_{\mathbf{h}}^{(1)} \wedge \mathbf{n}_{F_{\gamma}}\right) d \boldsymbol{\sigma}=\int_{T_{F_{\gamma}}} \mathbf{f} \cdot\left(\kappa_{F_{\gamma}} \mathbf{p}_{\mathbf{h}}^{(1)}\right) d \mathbf{x} .
\end{aligned}
$$

Using (6.93)-(6.96) in (6.92), it follows that

$$
\begin{aligned}
& \sum_{F_{\delta} \in \mathcal{F}_{h}\left(\delta_{m(j)}\right)}\left(\left\|\boldsymbol{\lambda}_{\mathbf{h}}-\left\{\mathbf{n} \wedge\left(\chi \operatorname{curl} \mathbf{j}_{\mathbf{h}} \wedge \mathbf{n}\right)\right\}\right\|_{0, F_{\delta}}^{2}+\left\|\left[\mathbf{n} \wedge\left(\chi \operatorname{curl} \mathbf{j}_{\mathbf{h}} \wedge \mathbf{n}\right)\right]\right\|_{0, F_{\delta}}^{2}\right) \\
& \leq C\left[\sum _ { T _ { F _ { \gamma } } \in \mathcal { T } _ { i } } \left(\int_{T_{F_{\gamma}}}\left(\mathbf{f}-\mathbf{f}_{\mathbf{h}}\right) \cdot\left(\kappa_{F_{\gamma}} \mathbf{p}_{\mathbf{h}}^{(1)}\right) d \mathbf{x}\right.\right. \\
& +\int_{T_{F \gamma}}\left(\mathbf{f}_{\mathbf{h}}-\operatorname{curl} \chi \operatorname{curl} \mathbf{j}_{\mathbf{h}}-\boldsymbol{\sigma} \mathbf{j}_{\mathbf{h}}\right) \cdot\left(\kappa_{F_{\gamma}} \mathbf{p}_{\mathbf{h}}^{(1)}\right) d \mathbf{x} \\
& \left.-\int_{T_{F_{\gamma}}}\left(\chi \operatorname{curl} \mathbf{e}_{\mathbf{j}} \cdot \operatorname{curl}\left(\kappa_{F_{\gamma}} \mathbf{p}_{\mathbf{h}}^{(1)}\right)+\boldsymbol{\sigma} \mathbf{e}_{\mathbf{j}} \cdot\left(\kappa_{F_{\gamma}} \mathbf{p}_{\mathbf{h}}^{(1)}\right)\right) d \mathbf{x}\right) \\
& +\sum_{T_{F_{\gamma}} \in \mathcal{T}_{j}} \int_{T_{F_{\delta}}}\left(\mathbf{f}-\mathbf{f}_{\mathbf{h}}\right) \cdot\left(\kappa_{F_{\delta}} \mathbf{p}_{\mathbf{h}}^{(2)}\right) d \mathbf{x} \\
& +\sum_{T_{F_{\delta}} \in \mathcal{T}_{j}}\left(\int_{T_{F_{\delta}}}\left(\mathbf{f}_{\mathbf{h}}-\operatorname{curl} \chi \operatorname{curl} \mathbf{j}_{\mathbf{h}}-\boldsymbol{\sigma} \mathbf{j}_{\mathbf{h}}\right) \cdot\left(\kappa_{F_{\delta}} \mathbf{p}_{\mathbf{h}}^{(2)}\right) d \mathbf{x}\right. \\
& \left.-\int_{T_{F_{\delta}}}\left(\chi \operatorname{curl} \mathbf{e}_{\mathbf{j}} \cdot \operatorname{curl}\left(\kappa_{F_{\delta}} \mathbf{p}_{\mathbf{h}}^{(2)}\right)+\boldsymbol{\sigma} \mathbf{e}_{\mathbf{j}} \cdot\left(\kappa_{F_{\delta}} \mathbf{p}_{\mathbf{h}}^{(2)}\right)\right) d \mathbf{x}\right) \\
& -\left.\sum_{F_{\delta} \in \mathcal{F}_{h}\left(\delta_{m(j)}\right)} \int_{F_{\delta}} \mathbf{e}_{\boldsymbol{\lambda}} \cdot\left(\kappa_{F_{\delta}} \mathbf{p}_{\mathbf{h}}^{(2)} \wedge \mathbf{n}_{F_{\delta}}\right)\right|_{\delta_{m(j)} \cap F_{\delta}} d \boldsymbol{\sigma} \\
& \left.-\left.\sum_{F_{\gamma} \in \mathcal{F}_{h}\left(\gamma_{m(i)}\right)} \int_{F_{\gamma}} \mathbf{e}_{\boldsymbol{\lambda}} \cdot\left(\kappa_{F_{\gamma}} \mathbf{p}_{\mathbf{h}}^{(1)} \wedge \mathbf{n}_{F_{\gamma}}\right)\right|_{\gamma_{m(i)} \cap F_{\gamma}} d \boldsymbol{\sigma}\right] .
\end{aligned}
$$

If we make use of the inverse inequality (6.68) and of (6.88)-(6.91), we get

$$
\begin{aligned}
\left(\sum_{F_{\delta} \in \mathcal{F}_{h}\left(\delta_{m(j)}\right)}\left(\left\|\boldsymbol{\lambda}_{\mathbf{h}}-\left\{\mathbf{n} \wedge\left(\chi \operatorname{curl} \mathbf{j}_{\mathbf{h}} \wedge \mathbf{n}\right)\right\}\right\|_{0, F_{\delta}}^{2}+\left\|\left[\mathbf{n} \wedge\left(\chi \operatorname{curl} \mathbf{j}_{\mathbf{h}} \wedge \mathbf{n}\right)\right]\right\|_{0, F_{\delta}}^{2}\right)\right)^{1 / 2} \\
\leq C\left[\sum_{T_{F_{\gamma}} \in \mathcal{T}_{i}} h_{T_{\gamma}}^{1 / 2}\left(\left\|\mathbf{f}-\mathbf{f}_{\mathbf{h}}\right\|_{T_{F_{\gamma}}}+\left\|\mathbf{f}_{\mathbf{h}}-\operatorname{curl} \chi \operatorname{curl} \mathbf{j}_{\mathbf{h}}-\boldsymbol{\sigma} \mathbf{j}_{\mathbf{h}}\right\|_{T_{F_{\gamma}}}\right)\right. \\
\quad+\sum_{T_{F_{\delta}} \in \mathcal{T}_{j}} h_{T_{\delta}}^{1 / 2}\left(\left\|\mathbf{f}-\mathbf{f}_{\mathbf{h}}\right\|_{T_{F_{\delta}}}+\left\|\mathbf{f}_{\mathbf{h}}-\operatorname{curl} \chi \operatorname{curl} \mathbf{j}_{\mathbf{h}}-\boldsymbol{\sigma} \mathbf{j}_{\mathbf{h}}\right\|_{T_{F_{\delta}}}\right) \\
\quad+\sum_{T_{F_{\gamma}} \in \mathcal{T}_{i}}\left(h_{T_{\gamma}}^{-1 / 2}\left\|\mathbf{e}_{\mathbf{j}}\right\|_{c u r l, T_{F_{\gamma}}}+h_{T_{\gamma}}^{1 / 2}\left\|\mathbf{e}_{\mathbf{j}}\right\|_{0, T_{F_{\gamma}}}\right)
\end{aligned}
$$




$$
\begin{aligned}
& +\sum_{T_{F_{\delta} \in \mathcal{T}_{j}}}\left(h_{T_{\delta}}^{-1 / 2}\left\|\mathbf{e}_{\mathbf{j}}\right\|_{\text {curl }, T_{F_{\delta}}}+h_{T_{\delta}}^{1 / 2}\left\|\mathbf{e}_{\mathbf{j}}\right\|_{0, T_{F_{\delta}}}\right) \\
& \left.+\sum_{F_{\gamma} \in \mathcal{F}_{h}\left(\gamma_{m(i)}\right)}\left\|\mathbf{e}_{\boldsymbol{\lambda}}\right\|_{0, F_{\gamma}}+\sum_{F_{\delta} \in \mathcal{F}_{h}\left(\delta_{m(j)}\right)}\left\|\mathbf{e}_{\boldsymbol{\lambda}}\right\|_{0, F_{\delta}}\right],
\end{aligned}
$$

from which (6.87) follows readily.

LEMMA 6.12. Under the same assumptions as in Lemma 6.11 let $\eta_{F_{\delta}}^{(\nu)}, 6 \leq \nu \leq$ 7 , be given by (6.9) and (6.10), respectively. Then, there holds

$$
\begin{aligned}
\left(\sum_{F_{\delta} \in \mathcal{F}_{h}\left(\delta_{m(j)}\right)}(\right. & \left.\left.\left(\eta_{F_{\delta}}^{(5)}\right)^{2}+\left(\eta_{F_{\delta}}^{(6)}\right)^{2}\right)\right)^{1 / 2} \\
\leq & C\left[\sum_{F_{\delta} \in \mathcal{F}_{h}\left(\delta_{m(j)}\right)}\left(\left\|\mathbf{e}_{\mathbf{j}}\right\|_{0, T_{F_{\delta}}}+\eta_{T_{F_{\delta}}}^{(2)}\right)\right. \\
& \left.+\sum_{F_{\gamma} \in \mathcal{F}_{h}\left(\gamma_{m(i)}\right)}\left(\left\|\mathbf{e}_{\mathbf{j}}\right\|_{0, T_{F_{\gamma}}}+\eta_{T_{F_{\gamma}}}^{(2)}\right)+\left\|e_{\lambda}\right\|_{M\left(\Gamma_{i j}\right)}\right] .
\end{aligned}
$$

Proof. Combining the ideas of proof used in Lemma 6.11 and those from the proof of (6.75) in Lemma 6.9 gives the assertion.

Proof of Theorem 6.2. The proof of (6.16) is a direct consequence of the local estimates provided by Lemmas $6.7-6.12$.

\section{Numerical Results}

7.1. Multigrid convergence rates for the eddy currents equations. As an academic test example, we consider the variational formulation of the eddy currents equations

$$
\int_{\Omega}\left(\mu^{-1} \operatorname{curl} \mathbf{j} \cdot \operatorname{curl} \mathbf{q}+\sigma \mathbf{j} \cdot \mathbf{q}\right) d \mathbf{x}=\int_{\Omega} \mathbf{f} \cdot \mathbf{q} d \mathbf{x} \quad, \quad \mathbf{q} \in \mathbf{H}_{\mathbf{0}}(\operatorname{curl} ; \Omega) .
$$

Here, the computational domain has been selected as $\Omega:=(-1,+1)^{3}$, and the righthand side $\mathbf{f}$ has been chosen such that $\mathbf{j}=\left(0,0, \sin \left(\pi x_{1}\right)\right)^{T}$ is the exact solution.

We have partitioned $\Omega$ into eight subdomains of equal size $\Omega_{\nu}, 1 \leq \nu \leq 8$. Starting from initial uniform simplicial triangulations $\mathcal{T}_{\nu}^{(0)}$ of $\Omega_{\nu}$ with $\overline{\mathcal{T}}_{2 \mu}^{(0)}$ being twice as fine as $\mathcal{T}_{2 \mu-1}^{(0)}, 1 \leq \mu \leq 4$, we have created three adaptive refinements by means of the residual-type a posteriori error estimator presented in section 6 .

In Table 7.1, for $\mu=1$ and different values of $\sigma$, convergence rates are listed for the multigrid iterative solution of the mortar edge element approximation. The columns contain the convergence rates for the preconditioned Richardson iteration without defect correction $\left(P R_{m g}\right)$ and with defect correction on the subspaces of irrotational vectorfields $\left(\right.$ Hybrid $1_{m g}$ and Hybrid $2_{m g}$ ). Here, Hybrid $1_{m g}$ refers to the hybrid smoother, where for the preconditioner non diagonal blocks have been used both for the unknowns in the interior of the subdomains and on the interfaces, whereas Hybrid $2_{m g}$ stands for the simplified hybrid smoother, where a non diagonal block is only used for the unknowns in the interior of the subdomains (cf. section $5)$.

As predicted by the theory, the multilevel iterative solution without defect correction on the subspaces of irrotational vector fields performs particularly bad 
TABLE 1. Convergence rates for the eddy currents equations

\begin{tabular}{|c|c|c|c|}
\hline $\boldsymbol{\sigma}$ & $P R_{m g}$ & Hybrid $1_{m g}$ & Hybrid $2_{m g}$ \\
\hline $10^{-4}$ & 0.999 & 0.665 & 0.699 \\
\hline $10^{-2}$ & 0.999 & 0.570 & 0.699 \\
\hline $10^{+0}$ & 0.940 & 0.565 & 0.677 \\
\hline $10^{+2}$ & 0.371 & 0.275 & 0.372 \\
\hline $10^{+4}$ & 0.477 & 0.464 & 0.475 \\
\hline
\end{tabular}

TABle 2. Performance of the error estimator for the eddy currents equations

\begin{tabular}{|c|r|r|c|}
\hline Level & \multicolumn{1}{|c|}{$E_{\text {ex }}$} & \multicolumn{1}{|c|}{$E_{\text {est }}$} & Eff \\
\hline 0 & $2.77 \mathrm{E}-2$ & $1.09 \mathrm{E}-1$ & 3.93 \\
\hline 1 & $1.62 \mathrm{E}-2$ & $7.16 \mathrm{E}-2$ & 4.42 \\
\hline 2 & $8.71 \mathrm{E}-3$ & $4.15 \mathrm{E}-2$ & 4.76 \\
\hline 3 & $4.45 \mathrm{E}-3$ & $2.18 \mathrm{E}-2$ & 4.90 \\
\hline
\end{tabular}

in case of small values of $\sigma$, where hybrid smoothing clearly pays off. Moreover, we see that the simplification of the preconditioner in the hybrid smoother only behaves slightly worse than hybrid smoothing with the original preconditioner. Therefore, considering the substantial savings in computational complexity, the simplified one appears to be the method of choice.

7.2. Performance of the residual-type a posteriori error estimator. For the same test example as before, we have tested the residual-type error estimator developed and analyzed in section 6. 
Table 7.1 shows the performance of the error estimator with $E_{\text {ex }}, E_{\text {est }}$ and Eff denoting the exact error, the estimated error, and the effectivity index, respectively.

7.3. Numerical computation of eddy currents in converter modules. We consider the computation of eddy currents in pulse width modulated converter modules that are used in high power electro motors for energy generation, energy transmission, and high technology transportation systems such as high speed trains. Figure 1 (left) shows the schematic representation of a converter module consisting of specific semiconductor devices such as Insulated Gate Bipolar Transistors (IGBTs) and Gate Turn Off Thyristors (GTOs).

The IGBTs and GTOs serve as valves for the electric currents in the range of several kiloampères featuring switching times of less than one hundred nanoseconds (cf. [16]). They are interconnected and linked with the power source and the electro motor by copper made bus bars. Figure 1 (right) displays the typical 3D configuration of a bus bar. Each bus bar has $N$ ports where the IGBTs and GTOs are attached to the device. Due to the high currents and fast switching times, eddy currents are generated in the bus bars leading to parasitic inductivities which may result in significant losses in the power transmission.
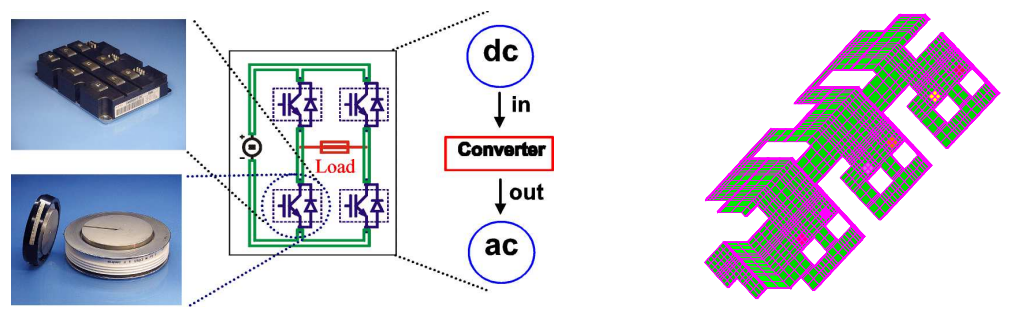

FiguRE 1. Schematic representation of a converter module (left) and typical 3D configuration of a copper made bus bar with ports (right)

In the sequel, we restrict ourselves to a single bus bar occupying a bounded domain $\Omega \subset \mathbb{R}^{3}$ with $N$ ports $\Gamma_{\nu} \subset \Gamma, 1 \leq \nu \leq N$. The operational behavior of a bus bar can be described by the eddy currents equations which we consider in its potential formulation by means of a scalar electric potential $\varphi$ and a magnetic vector potential $\mathbf{A}$ which satisfy a coupled system of PDEs consisting of an elliptic boundary value for the scalar electric potential in $\Omega$

$$
\begin{gathered}
\operatorname{div}(\varepsilon \operatorname{grad} \varphi)=0 \text { in } \Omega, \\
\mathbf{n} \cdot \boldsymbol{\sigma} \operatorname{grad} \varphi=\left\{\begin{array}{cc}
-I_{\nu}(t) & \text { on } \Gamma_{\nu} \\
0 & \text { else }
\end{array}\right.
\end{gathered}
$$

and a parabolic equation for the double curl operator

$$
\boldsymbol{\sigma} \frac{\partial \mathbf{A}}{\partial t}+\operatorname{curl} \chi \operatorname{curl} \mathbf{A}=\left\{\begin{array}{cc}
-\sigma \operatorname{grad} \varphi & \text { in } \Omega \\
0 & \text { in } \mathbf{R}^{3} \backslash \Omega
\end{array},\right.
$$

which has to be considered in the interior and exterior domain together with appropriate boundary, transmission, and initial conditions. Here, $I_{\nu}, 1 \leq \nu \leq N$, are 
the current densities at the contacts satisfying the compatibility condition

$$
\sum_{\nu=1}^{N} I_{\nu}(t)=0
$$

We have used a decomposition of the computational domain into an interior domain, i.e., the domain occupied by the bus bar, and an exterior domain with an artificial boundary off the bus bar. The interior domain has been further subdivided according to the geometry of the bus bar.

For the discretization of the potential equation $(7.2),(7.3)$ for the scalar electric potential $\varphi$ in the subdomains making up the interior domain, we have implemented the lowest order nonconforming Crouzeix-Raviart elements. The reason for this is that in subsequent work the numerical solution of the state equations has been used as an integral part in the topology optimization of the bus bar (cf., e.g., [42-44]). In contrast to standard conforming P1 elements, the use of nonconforming P1 elements avoids unwanted checkerboarding in the design. For the discretization in time of the parabolic equation (7.4) for the magnetic vector potential $\mathbf{A}$ we have used the backward Euler scheme, whereas the discretization in space both in the interior and the exterior domain has been done by means of the lowest order curl-conforming edge elements of Nédélec's first family. Note that $\boldsymbol{\sigma}=\mathbf{0}$ in the exterior domain and therefore, we have enforced a gauge condition.

At each time step, the iterative solution of the discretized state equations (7.2)(7.4) has been realized by mortar edge element methods as described in sections 4-6 of this paper.
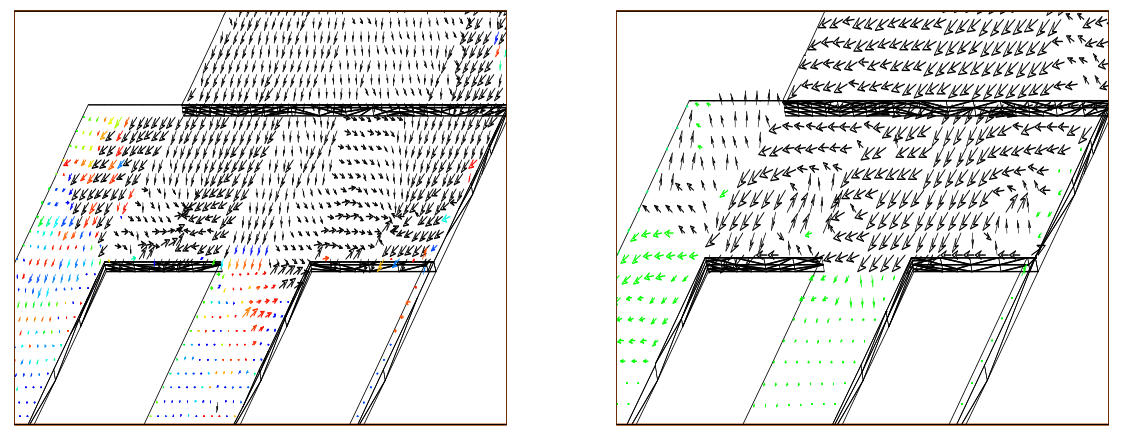

FiguRE 2. Visualization the magnetic vector potential (left) and of the magnetic flux (right) in the vicinity of two ports

Figure 2 displays the computed magnetic vector potential $\mathbf{A}$ (left) and the magnetic field $\mathbf{B}=$ curl $\mathbf{A}$ (right) in a vicinity of two ports that are located above the two holes in the lower part of the pictures.

7.4. Numerical simulation of Logging-While-Drilling (LWD) tools.

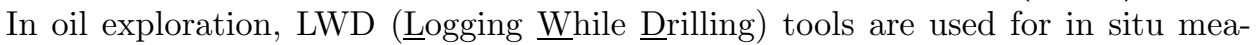
surements to provide information about the formation through which a borehole is drilled. They acquire data from sensors integrated into the drill string and thus allow data recording at almost the same time when the hole is cut. LWD tools are induction sensors with several saddle-type transmitter and receiver antennas 
placed concentrically on a metallic mandrel (cf. Figure 3 (left). The antennas are approximately cylindrically symmetric. Figure 3 (right) shows a cut through the antenna at a fixed azimuth. The radio frequency magnetic field is created by an azimuthal current sheet, realized by a coil wrapped around the mandrel. Since the magnetic flux cannot penetrate into the mandrel, behind the coil a recess is cut into the mandrel to allow the flux to pass. Moreover, for protection from the drilling environment, the antenna is covered by a shielding.
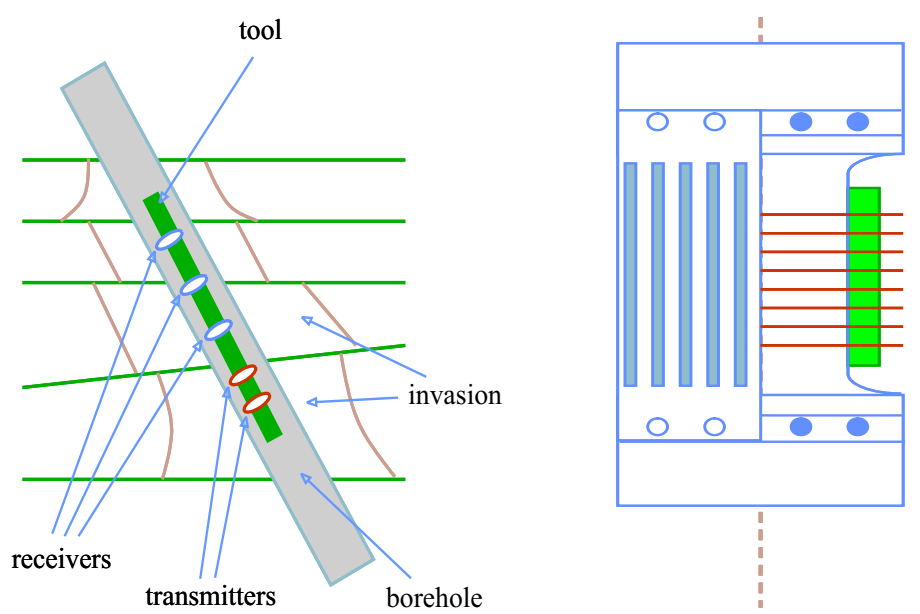

FiguRE 3. Schematic representation of an LWD tool (left) and fine details of a saddle antenna (right)

The entire computational domain $\Omega$ consists of that part of the mandrel $\Omega_{1}$ and the borehole $\Omega_{2}$, where the transmitter and receiver antennas are located, and a neighboring part of the surrounding formation $\Omega_{3}$ with an artificial boundary sufficiently far off the borehole. The domain $\Omega_{1}$ is further partitioned into subdomains such that each subdomain contains exactly one transmitter or receiver antenna. The electromagnetic field generated by the transmitter currents can be described by the complex time-harmonic Maxwell equations with impedance boundary conditions on $\Gamma_{12}:=\bar{\Omega}_{1} \cap \bar{\Omega}_{2}$, transmission boundary conditions on $\Gamma_{23}:=\bar{\Omega}_{2} \cap \bar{\Omega}_{3}$, and homogeneous Dirichlet boundary conditions (vanishing tangential components traces) elsewhere. Denoting by $\mathbf{E}_{\mathbf{R}}$ and $\mathbf{E}_{\mathbf{I}}$ the real and the imaginary part of the electric field $\mathbf{E}$, the variational formulation gives rise to the system of equations

$$
\begin{aligned}
& \int_{\Omega}\left(\mu^{-1} \operatorname{curl} \mathbf{E}_{\mathbf{R}} \cdot \operatorname{curl} \mathbf{q}-\omega^{2} \varepsilon \mathbf{E}_{\mathbf{R}} \cdot \mathbf{q}\right) d \mathbf{x} \\
&-<\omega\left(\kappa_{I} \gamma_{\mathbf{t}}\left(\mathbf{E}_{\mathbf{R}}\right)+\kappa_{R} \gamma_{\mathbf{t}}\left(\mathbf{E}_{\mathbf{I}}\right)\right), \boldsymbol{\pi}_{\mathbf{t}}(\mathbf{q})>_{1 / 2, \Gamma_{12}}+\int_{\Omega} \omega \sigma \mathbf{E}_{\mathbf{I}} \cdot \mathbf{q} d \mathbf{x} \\
&=-\int_{\Omega} \omega \mathbf{j}_{\mathbf{I}} \cdot \mathbf{q} d \mathbf{x},
\end{aligned}
$$


(7.6)

$$
\begin{aligned}
\int_{\Omega}\left(\mu^{-1} \operatorname{curl} \mathbf{E}_{\mathbf{I}} \cdot \operatorname{curl} \mathbf{q}-\omega^{2} \varepsilon \mathbf{E}_{\mathbf{I}} \cdot \mathbf{q}\right) d \mathbf{x} & \\
-<\omega\left(\kappa_{I} \gamma_{\mathbf{t}}\left(\mathbf{E}_{\mathbf{I}}\right)+\kappa_{R} \gamma_{\mathbf{t}}\left(\mathbf{E}_{\mathbf{R}}\right)\right), \boldsymbol{\pi}_{\mathbf{t}}(\mathbf{q})>_{1 / 2, \Gamma_{12}} & -\int_{\Omega} \omega \sigma \mathbf{E}_{\mathbf{R}} \cdot \mathbf{q} d \mathbf{x} \\
& =-\int_{\Omega} \omega \mathbf{j}_{\mathbf{R}} \cdot \mathbf{q} d \mathbf{x},
\end{aligned}
$$

where $\omega$ is the frequency and $\kappa_{R}, \kappa_{I}$ stand for the real and imaginary part of $\kappa=(1+i) \sqrt{(\pi \sigma) /(\omega \mu)}$.
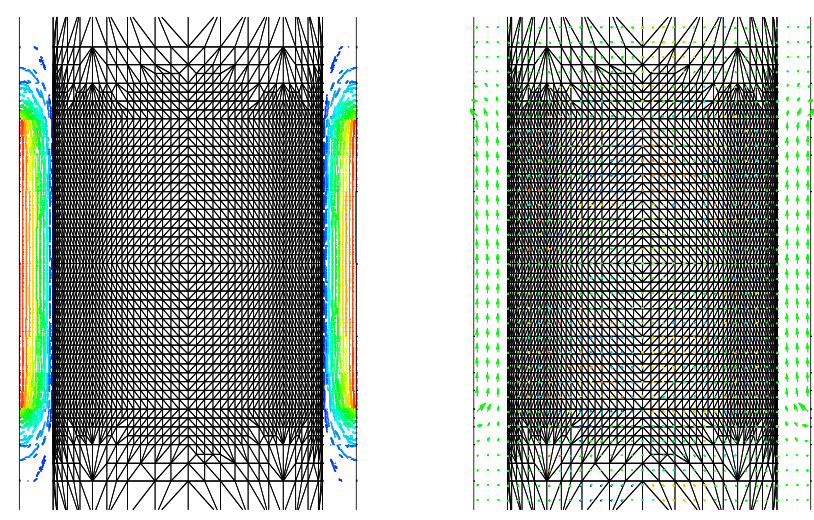

FIGURE 4. Adaptively generated grid and visualization of the electric field (left) and the magnetic field (right) in the vicinity of the transmitter antenna

The adaptive multilevel algorithm based on the mortar edge element approximation as described in sections 4-6 has been applied to a benchmark problem with one transmitter and two receiver antennas. The borehole and the mandrel are circular cylinders of diameters 8 and 1.6 inches, respectively. The saddle antennas are placed at axial positions with a distance of 18 inches to each other. The height of each saddle antenna is 4 inches, the radius is 1.2 inches, and the aperture angle is $90^{\circ}$. The transmitter antenna carries a current of $1 A$ and the frequency is $\omega=54 \mathrm{kHz}$. All materials are assumed to have a relative permittivity and relative permeability of 1 . The conductivities of the mandrel, the mud inside the borehole, and the formation outside the borehole are assumed to be $10^{7} \mathrm{~S} / \mathrm{m}, 10 \mathrm{~S} / \mathrm{m}$ and $0.05 S / m$,respectively.

Figure 4 displays the adaptively generated grid and a visualization of the computed electric field (left) and magnetic field (right).

Acknowledgements. This work has been supported by the German Federal Ministry for Education and Research (BMBF) under Grants No. 03HO07AU1, No. 03HOM3A1, and by the German National Science Foundation (DFG) under Grant No. HO87774-2. 


\section{References}

1. M. Ainsworth and J. Coyle, Hierarchic hp-edge element families for Maxwell's equations on hybrid quadrilateral/triangular meshes, Comp. Meth. Appl. Mech. Engrg. 190 (2001), 67096733.

2. M. Ainsworth and A Oden, Posteriori error estimation in finite element analysis, Wiley, Chichester, 2000.

3. A. Alonso and A. Valli, Some remarks on the characterization of the space of tangential traces of $h(r o t ; \omega)$ and the construction of an extension operator, Manuscr. Math. 89 (1996), $159-178$.

4. __ An optimal domain decomposition preconditioner for low-frequency time harmonic Maxwell equations, Math. Comp. 68 (1999), 607-631.

5. D. Arnold, R. Falk, and R. Winther, Multigrid in H(div) and H(curl), Numer. Math. 85 (2000), 197-218.

6. I. Babuska and T. Strouboulis, The finite element method and its reliability, Clarendon Press, Oxford, 2001.

7. W. Bangerth and R. Rannacher, Adaptive finite element methods for differential equations, Lectures in Mathematics, ETH-Zürich. Birkhäuser, Basel, 2003.

8. R. Beck, P. Deuflhard, R. Hiptmair, R.H.W Hoppe, and B. Wohlmuth, Adaptive multilevel methods for edge element discretizations of Maxwell's equations, Surveys of Math. in Industry 8 (1999), 271-312.

9. R. Beck, R. Hiptmair, R.H.W. Hoppe, and B. Wohlmuth, Residual based a posteriori error estimators for eddy current computation, $\mathrm{M}^{2} \mathrm{AN}$ Math. Modeling and Numer. Anal. 34 (2000), 159-182.

10. R. Beck, R. Hiptmair, and B. Wohlmuth, Hierarchical error estimator for eddy current computation, Proc. 2nd European Conf. on Advanced Numer. Meth. (ENUMATH99) (Singapore) (P. Neittaanmäki et al., ed.), Jyväskylä, Finland, July 26-30, 1999, World Scientific, 2000, pp. 111-120.

11. A. Ben Abdallah, F. Ben Belgacem, and Y. Maday, Mortarting the two-dimensional nédélec finite elements for the discretization of the maxwell equations, to appear in $\mathrm{M}^{3} \mathrm{AS}, 2003$.

12. F. Ben Belgacem, A. Buffa, and Y. Maday, The mortar finite element method for $3 d$ Maxwell equations: first results, SIAM J. Numer. Anal. 39 (2001), 880-901.

13. C. Bernardi, Y. Maday, and A. Patera, Domain decomposition by the mortar element method, Asymptotic and Numerical Methods for Partial Differential Equations with Critical Parameters (H. Kaper et al., ed.), Reidel, Dordrecht, 1993, pp. 269-286.

14. _ A new nonconforming approach to domain decomposition: the mortar element method, Nonlinear partial differential equations and their applications (H. Brezis et al., ed.), Paris, 1994, pp. 13-51.

15. D. Boffi, L. Brezzi, and L. Gastaldi, Mixed finite elements for Maxwell's eigenproblem: the question of spurious modes, Proc. 2nd European Conference on Numerical Methods (ENUMATH 97) (Singapore) (H.G. Bock, F. Brezzi, R. Glowinski, G. Kanschat, Y. Kuznetsov, J. Périaux, and R. Rannacher, eds.), World Scientific, 1998, pp. 180-187.

16. P. Böhm, R.H.W. Hoppe, G. Mazurkevitch, S. Petrova, G. Wachutka, and E. Wolfgang, Optimal structural design of high power electronic devices by topology optimization, Mathematics - Key Technology for the Future. Cooperations between Mathematics and Industry (H. Krebs and W. Jäger, eds.), Springer, Berlin-Heidelberg-New York, 2003, pp. 365-376.

17. A. Bossavit, Solving Maxwell's equations in a closed cavity and the question of spurious modes, IEEE Trans. Magn. 26 (1990), 702-705.

18. __ Electromagétisme, en vue de la modélisation, Springer, Paris, 1993.

19. _ Computational electromagnetism. variational formulation, complementarity, edge elements, Academic Press, San Diego, 1998.

20. D. Braess and W. Dahmen, Stability estimates of the mortar finite element method for 3dimensional problems, East-West J. Numer. Math. 6 (1999), 249-264.

21. D. Braess, W. Dahmen, and C. Wieners, A multigrid algorithm for the mortar finite element method, SIAM j. Numer. Anal. 37 (2000), 48-69.

22. J.H. Bramble, Multigrid methods, Addison Wesley Longman, 1993.

23. F. Brezzi and M. Fortin, Mixed and hybrid finite element methods, Springer, BerlinHeidelberg-New York, 1991. 
24. A. Buffa and Ph. Jr. Ciarlet, On traces for functional spaces realted to Maxwell's equations. part I: An integration by parts formula in Lipschitz polyhedra, Math. Meth. Appl. Sci. 24 (2001), 9-30.

25. - On traces for functional spaces realted to Maxwell's equations. part II: Hodge decompositions on the boundary of Lipschitz polyhedra and applications, Math. Meth. Appl. Sci. 24 (2001), 31-48.

26. A. Buffa, M. Costabel, and D. Sheen, On traces for h(curl, $\omega)$ in Lipschitz domains, J. Math. Anal. Appl. 276 (2002), 845-867.

27. A. Buffa, Y. Maday, and F. Rapetti, A sliding mesh-mortar method for a two-dimensional eddy currents model of electric engines, Math. Model. Numer. Anal. 35 (2001), 191-228.

28. N. Debit, M. Garbey, R.H.W. Hoppe, D. Keyes, Y.A. Kuznetsov, and J. Périaux, Domain decomposition methods in science and engineering, Proc. 13th Int. Conf. on Domain Decomposition Methods (Barcelona), Handbooks on Theory and Engrg. Appl. of Comput. Methods, Lyon, October 2001, CIMNE, 2002.

29. B. Engelmann, R.H.W. Hoppe, Y. Iliash, Y. Kuznetsov, Y. Vassilevski, and B. Wohlmuth, Adaptive macro-hybrid finite element methods, Proc. 2nd European Conference on Numerical Methods (ENUMATH 97) (Singapore) (H.G. Bock, F. Brezzi, R. Glowinski, G. Kanschat, Y. Kuznetsov, J. Périaux, and R. Rannacher, eds.), Heidelberg, Sept. 29 - Oct. 3, 1997, World Scientific, 1998, pp. 294-302.

30. _ Adaptive finite element methods for domain decomposition on nonmatching grids, Parallel Solution of PDEs (Berlin-Heidelberg-New York) (P. Bjørstad and M. Luskin, eds.), IMA Volume in Mathematics and its Applications, vol. 120, Springer, 2000, pp. 57-83.

31. K. Eriksson, D. Estep, P. Hansbo, and C. Johnson, Computational differential equations, Cambridge University Press, Cambridge, 1996.

32. B. Flemisch, Y. Maday, F. Rapetti, and B. Wohlmuth, Coupling scalar and vector potentials on non-matching grids for eddy current modelling in moving conductors, J. Comput. Appl. Math. 168 (2004), 192-205.

33. V. Girault and P.A. Raviart, Finite element approximatiuon of the Navier-Stokes equations, Lecture Notes in Mathematics, vol. 749, Springer, Berlin-Heidelberg-New York, 1979.

34. G. Haase, M. Kuhn, and U. Langer, Parallel multigrid 3d maxwell solvers, Parallel Computing 27 (2001), 761-775.

35. W. Hackbusch, Multi-grid methods and applications, Springer, Berlin-Heidelberg-New York, 1985.

36. R. Hiptmair, Multigrid method for Maxwell's equations, SIAM J. Numer. Anal. 36 (1998), 204-225.

37. __ Finite elements in computational electromagnetism, Acta Numerica 11 (2002), 237339.

38. R.H.W. Hoppe, Mortar edge elements in $r^{3}$, East-West J. Numer. Anal. 7 (1999), 159-173.

39. __ Adaptive domain decomposition techniques in electromagnetic field computation and electrothermomechanical coupling problems, Proc. 4th European Conference on Numerical Mathematics and Advanced Applications (Berlin-Heidelberg-New York) (F. Brezzi et al., ed.), Ischia, Italy, July 23-27, 2001, Springer, 2002.

40. Adaptive multigrid and domain decomposition methods in the computation of electromagnetic fields, J. Comput. Appl. Math. 168 (2004), 245-254.

41. R.H.W. Hoppe, Y. Iliash, Y. Kuznetsov, Y. Vassilevski, and B. Wohlmuth, Analysis and parallel implementation of adaptive mortar element methods, East-West J. Numer. Math. 6 (1998), 223-248.

42. R.H.W. Hoppe and S. Petrova, Primal-dual Newton interior-point methods in shape and topology optimization, Numerical Linear Algebra with Applications 11 (2004), 413-429.

43. R.H.W. Hoppe, S. Petrova, and V. Schulz, A primal-dual Newton-type interior-point method for topology optimization, Journal of Optimization, Theory and Applications 114 (2002), $545-571$.

44. Topology optimization of high power electronic devices, Proc. Oberwolfach Conference "Optimal Control and Optimization" (G. Leugering et al., ed.), Int. Series in Numer. Math., vol. 139, Oberwolfach, June 5-9, 2000, 2002, pp. 119-131.

45. P. Houston, I. Perugia, and D. Schötzau, Mixed discontinuous galerkin approximation of the Maxwell operator, SIAM J. Numer. Anal. 42 (2004), 434-459. 
46. R. Kornhuber, R.H.W. Hoppe, D. Keyes, J. Périaux, O. Pironneau, and J. Xu, Domain decomposition methods and applications, to appear in Proc. 15th Int. Conf. on Domain Decomposition Methods, Berlin, July 2003, Springer, Berlin-Heidelberg-New York, 2004.

47. Y.A. Kuznetsov, Efficient iterative solvers for elliptic finite element problems on nonmatching grids, Russian J. Numer. Anal. Math. Modeling 10 (1995), 187-212.

48. P. Monk, Finite element methods for Maxwell's equations, Clarendon Press, Oxford, 2003.

49. J.-C. Nédélec, Mixed finite elements in $\mathbb{R}^{3}$, Numer. Math. 35 (1980), 315-341.

50. _ A new family of mixed finite elements in $\mathbb{R}^{3}$, Numer. Math. 50 (1986), 57-81.

51. I. Perugia, D. Schötzau, and P. Monk, Stabilized interior penalty methods for the timeharmonic Maxwell equations, Comp. Meth. Appl. Mech. Engrg. 191 (2002), 4675-4697.

52. A. Quarteroni and A. Valli, Domain decomposition methods for partial differential equations, Clarendon Press, Oxford, 1999.

53. W. Rachowicz and L. Demkowicz, An hp-adaptive finite element method for electromagnetics. part I: Data structure and constrained approximation, Comp. Meth. Appl. Mech. Engrg. 187 (2000), 307-337.

54. __ An hp-adaptive finite element method for electromagnetics. part II: A 3D implementation, Int. J. Numer. Meth. Engrg. 53 (2002), 147-180.

55. F. Rapetti, Approximation des equations de la magnetodynamique en domaine tournant par la méthode des elements avec joints, Ph.D. thesis, Université Pierre et Marie Curie, Paris, 2000.

56. in moving structures, Int. J. Numer. Mod. 14 (2001), 457-477.

57. F. Rapetti, A. Buffa, Y. Maday, and F. Bouillault, Simulation of a coupled magnetomechanical system through the sliding-mesh mortar element method, COMPEL 19 (2000), $332-340$.

58. F. Rapetti, Y. Maday, and F. Bouillault, Eddy current calculations in three-dimensional structures, IEEE Trans. Magnetics 38 (2002), 613-616.

59. S. Reitzinger and J. Schöberl, Algebraic multigrid for edge elements, Numer. Lin. Alg. Appl. 9 (2002), 223-238.

60. L.R. Scott and Z. Zhang, Finite element interpolation of nonsmooth functions satisfying boundary conditions, Math. Comp, 54 (1990), 483-493.

61. B.F. Smith, P.E. Bjørstad, and W.D. Gropp, Domain decomposition methods, Cambridge University Press, Cambridge, 1996.

62. A. Toselli and A. Klawonn, A FETI domain decomposition method for edge element approximations in two dimensions with discontinuous coefficients, SIAM J. Numer. Anal. 39 (2001), 932-956.

63. A. Toselli and X. Vasseur, Dual-primal FETI-algorithms for edge element approximations: two-dimensional $h$ and $p$ finite elements on shape-regular meshes, submitted to SIAM J. Numer. Anal., 2004.

64. R. Verfürth, A review of a posteriori estimation and adaptive mesh-refinement techniques, Wiley-Teubner, New York, Stuttgart, 1996.

65. M.N. Vouvakis and J.-F. Lee, A fast DP-FETI like domain decomposition algorithm for the solution of large electromagnetic problems, to appear in Proc. 8th Copper Mountain Conf. on Iterative Methods, Copper Mountain, Colorado, March 28 - April 2, 2004.

66. P. Wesseling, An introduction to multigrid methods, Wiley, Chichester, 1992.

67. B. Wohlmuth, A mortar finite element method using dual spaces for the Lagrange multiplier, SIAM J. Numer. Anal. 38 (2000), 989-1012.

68. _ Discretization methods and iterative solvers based on domain decomposition, Lecture Notes in Computational Science and Engineering, vol. 17, Springer, Berlin-Heidelberg-New York, 2001.

69. X. Xu and R.H.W. Hoppe, On the convergence of mortar edge element methods in $\mathbb{R}^{3}$, Preprint, Inst. of Math., University of Augsburg, submitted to SIAM J. Numer. Anal., 2004.

70. Berlin-Heidelberg-New York (ed.), Computational electromagnetics, Lecture Notes in Computational Science and Engineering, vol. 28, Springer, 2003.

71. K. Zhao, M.N. Vouvakis, S.-C. Lee, and J.-F. Lee, Domain decomposition method in conjunction with DP-FETI for modeling large finite arrays, to appear in Proc. in Electromagnetics Research Symp. (PIERS 2004), Nanjing, China, August 28-31, 2004. 
Department of Mathematics, University of Houston, Houston, TX 77204-3008, USA And, Institute of Mathematics, University of Augsburg, D-86159 Augsburg, Germany

E-mail address: rohop@math.uh.edu 